\title{
Millions of standard models on $\mathbb{Z}_{6}^{\prime}$ ?
}

\author{
Florian Gmeiner \\ NIKHEF, \\ Kruislaan 409, 1098 SJ Amsterdam, The Netherlands \\ E-mail: fgmeiner@nikhef.n]
}

\section{Gabriele Honecker}

PH-TH Division, CERN,

1211 Geneva 23, Switzerland

E-mail: Gabriele.Honecker@cern.ch

ABSTRACT: Using previous results on the statistics of intersecting D6-brane models on $T^{6} / \mathbb{Z}_{6}^{\prime}$ we analyse solutions that resemble the gauge group and matter content of the supersymmetric standard model. In particular the structure of the hidden sector, the gauge coupling constants and chiral exotic matter content are computed and classified for all possible standard model-like configurations on this background. It turns out that the number of chiral exotics, Higgses and values of gauge couplings are strongly correlated. Some examples with the chiral spectrum of the supersymmetric standard model plus vectorlike states and a massless $\mathrm{U}(1)_{B-L}$ are discussed in detail.

KEYWORDS: 〔ntersecting branes models, Superstring Vacua. 


\section{Contents}

1. Introduction 2

2. Set-up 3

3. Complete massless spectrum 5

3.1 Closed string spectrum

3.2 Massless open spectrum from three-cycle intersection numbers 6

3.2.1 For branes at three non-vanishing angles 6

3.2.2 For branes parallel along all three tori

3.2.3 For branes parallel along the $\mathbb{Z}_{2}$ invariant $T_{2}^{2}$

3.2.4 For branes parallel along $T_{3}^{2}$ where $\mathbb{Z}_{2}$ acts non-trivially 9

3.3 Massless spectrum from Chan-Paton matrices 10

3.3.1 Branes parallel on all tori 11

3.3.2 Branes at non-trivial angles 11

3.3.3 Branes parallel along the $\mathbb{Z}_{2}$ invariant torus 13

3.3.4 Branes parallel along a torus where $\mathbb{Z}_{2}$ acts

4. Gauge couplings 16

5. Standard model constraints 18

6. Statistics 20

6.1 Relation between solutions on different geometries 20

6.2 Counting standard model-like solutions 20

6.3 Complex structure dependence 20

6.4 Gauge couplings 22

6.5 Number of Higgs multiplets 24

6.6 Chiral exotics 24

6.6.1 Correlation between "chiral" Higgs multiplets and chiral exotics 26

6.7 Models without chiral exotics 26

6.7.1 Visible sector 27

6.7.2 Hidden sector 28

6.7 .3 Adjoint representations 30

7. Explicit standard model-like realisations 30

7.1 Three generation models with hidden sectors 30

7.2 A three generation model without hidden sector 36

8. Conclusions 39

A. Chan-Paton labels 40 


\section{Introduction}

Understanding the structure of the large amount of string theory compactifications, also known as the landscape, is one of the big challenges for string phenomenology. In order to make contact with physics at accessible energy scales, it is necessary to explore all feasible ways to obtain a standard model-like theory, a task that is complicated by the huge amount of possibilities. A deeper understanding of patterns in the space of solutions could lead not only to a more refined string model building, but if we are lucky to direct predictions based on a statistical analysis of correlations in the landscape.

In recent years, a large number of string theory vacua has been found by using a statistical approach, see e.g. [1] for heterotic orbifolds, [2] for free fermionic models, [3] for rational conformal field theories and 国-11] for intersecting D-branes in orbifold backgrounds. For the latter examples, CFT techniques have recently been applied to compute non-perturbative Yukawa couplings and Majorana mass terms, see e.g. [12, 13], which can in particular lead to non-vanishing results for instantons on rigid cycles. However, to our knowledge, no fully-fledged intersecting brane model with standard model spectrum on rigid cycles has been found so far. ${ }^{1}$

In this article, we focus on properties and correlations of the intersecting D6-brane models on $T^{6} / \mathbb{Z}_{6}^{\prime}$ found in [1]] (for the set-up see also [18-20]) with supersymmetric standard model-like spectra. In contrast to the smooth heterotic $E_{8} \times E_{8}$ compactifications of, e.g., [21], intersecting D6-brane models in $T^{6} / \mathbb{Z}_{2 N}$ backgrounds are built on fractional but non-rigid branes and do always have some amount of non-chiral matter states, in particular chiral multiplets in the adjoint representation. We use a combined method of three-cycle intersection numbers and Chan-Paton matrices to determine the full massless spectrum needed to compute the one-loop beta function coefficients of the gauge couplings. Due to the large number of $\mathcal{O}\left(10^{23}\right)$ supersymmetric RR tadpole solutions, our focus in the present article is on a statistical evaluation of supersymmetric standard model-like string spectra, in particular the subset of $\mathcal{O}\left(10^{7}\right)$ models without chiral exotic matter. While the bigger set of around $\mathcal{O}\left(10^{15}\right)$ three generation models displays a large variety of abundances of chiral exotics and "chiral" Higgs candidates, hidden sectors as well as diverse ratios of gauge couplings, it turns out that these quantities are strongly correlated. Our analysis is performed for one out of two possible background geometries for which we prove the

\footnotetext{
${ }^{1}$ See e.g. $\sqrt{14}-17$ for model searches on $T^{6} / \mathbb{Z}_{2} \times \mathbb{Z}_{2}$ with torsion and rigid cycles.
} 
equivalence of bulk solutions. Some examples of spectra with exactly three quark-lepton generations plus vector-like states and a massless $B-L$ symmetry are discussed in detail. Selection rules for perturbative Yukawa couplings are considered as well as those mass terms which can arise through parallel displacement of branes.

The methods to analyse the ensemble of solutions to the supersymmetry and RR tadpole equations for models on a particular orbifold background have been developed in an earlier publication [11] and are based on the general idea to classify large amounts of models in the landscape. While this approach has shown to be useful to gain insights into the structure of the space of solutions, there are some caveats not to be overlooked. In particular, one has to be very careful which solutions to the equations one wants to count as individual models and which are just different realisations of the same low energy theory that might be related by a symmetry. Moreover, by using a statistical approach there exists the possibility that one finds correlations between properties of the solutions that are artefacts of the method by which the solutions are generated [22]. We do not have to worry about the second issue in the case at hand, since we construct all possible solutions explicitly (although we do not analyse every single solution in full detail) and thereby can be sure that no unwanted bias is introduced. With respect to the first issue, we are providing a careful analysis of the geometric set-up, but since not all solutions could be checked for the complete non-chiral matter content, there remains an uncertainty about the true value of completely unrelated models.

The paper is organised as follows. In section 2 we review the geometric set-up and consistency conditions for intersecting D-brane models in the $T^{6} / \mathbb{Z}_{6}^{\prime}$ orientifold background. In section 3 we determine the complete massless spectrum using a combined approach of intersection numbers and Chan-Paton matrices with special emphasis on the cases where branes are parallel along some direction, clarifying in particular the cases with symmetric and antisymmetric representations. In section \$, tree level gauge couplings and the oneloop running due to massless string modes are discussed, and in section 5 our constraints on standard model-like spectra are given. In section 6, the results of the statistical analysis are presented with some standard model-like examples without chiral exotics discussed in detail in section 8. Our conclusions are given in section 8, and finally technical details are collected in appendices $\mathrm{A}$ to $\mathrm{D}$, and the existence of trinification spectra is debated in appendix 国.

\section{Set-up}

The set-up of the $T^{6} / \mathbb{Z}_{6}^{\prime}$ orientifold with fractional D6-branes has been discussed in detail in [11..$^{2}$ We briefly review the main features here. The $\mathbb{Z}_{6}^{\prime}$ orbifold generator acts as

$$
\theta: z^{k} \rightarrow e^{2 \pi i v_{k}} z^{k} \quad \text { with } \quad \vec{v}=\frac{1}{6}(1,2,-3)
$$

\footnotetext{
${ }^{2}$ Our choice of a basis of three-cycles differs from the one in [18-20].
} 
on the complex coordinates $z^{k}(k=1,2,3)$ of a factorised six-torus $T_{1}^{2} \times T_{2}^{2} \times T_{3}^{2}{ }^{3}$ The geometric part $\mathcal{R}$ of the orientifold action $\Omega \mathcal{R}$,

$$
\mathcal{R}: z^{k} \rightarrow \bar{z}^{k}
$$

enforces the $\mathrm{SU}(3) \times \mathrm{SU}(3) \times \mathrm{SU}(2)^{2}$ lattice to have one of two possible orientations $\mathbf{A}$ or $\mathbf{B}$ per $\mathrm{SU}(3)$ and $\mathbf{a}$ or $\mathbf{b}$ per $\mathrm{SU}(2)^{2}$ lattice with respect to the $\operatorname{Re}\left(z^{k}\right)$ directions. The $\mathbf{a}$ and $\mathbf{b}$ torus are conveniently parameterised by $b=0,1 / 2$, respectively. The $T^{6} / \mathbb{Z}_{6}^{\prime}$ orbifold has 24 three-cycles with four, $\rho_{i}(i=1 \ldots 4)$, stemming from the underlying torus, eight, $\delta_{j}, \tilde{\delta}_{j}(j=1 \ldots 4)$, associated to the $\mathbb{Z}_{2}$ sub-symmetry and twelve more pertaining to the $\mathbb{Z}_{3}$ sub-symmetry. The former two kinds $\left(\rho_{i}, \delta_{j}, \tilde{\delta}_{j}\right)$ form a twelve dimensional sub-lattice from which fractional cycles

$$
\begin{aligned}
\Pi^{\mathrm{frac}} & =\frac{1}{2} \Pi^{\mathrm{bulk}}+\frac{1}{2} \Pi^{\mathrm{ex}} \\
& =\frac{1}{2}\left(\sum_{i=1}^{4} \tilde{a}_{i} \rho_{i}+\sum_{j=1}^{4}\left(d_{j} \delta_{j}+e_{j} \tilde{\delta}_{j}\right)\right)
\end{aligned}
$$

are built with coefficients $\tilde{a}_{i}, d_{j}, e_{j} \in \mathbb{Z}$ which are composed of toroidal one-cycle wrapping numbers $\left(n_{i}, m_{i}\right)$ discussed in more detail in appendix D. ${ }^{4}$

The RR tadpole cancellation conditions can be written in the general form

$$
\sum_{a} N_{a} \vec{X}_{a}=\vec{L}
$$

with $\vec{L}=\left(L_{1}, L_{2}, 0,0,0,0\right)^{T}$ and the bulk entries $X_{1}, X_{2}$ listed in table 1 descending from the toroidal cycles. The contributions from exceptional cycles, $X_{3} \ldots X_{6}$, are given in appendix D in equations (D.9) and (D.10).

The supersymmetry condition for the toroidal part of a three-cycle $\Pi^{\text {frac }}$ can be cast into the form

$$
\vec{Y} \cdot \vec{F}(U)=0, \quad \vec{X} \cdot \vec{U}>0,
$$

with the bulk coefficients given in table 1 and

$$
\begin{aligned}
\left(\begin{array}{c}
U_{1} \\
U_{2}
\end{array}\right) & \sim\left(\begin{array}{c}
1 \\
c_{1}
\end{array}\right) \quad \text { with } \quad c_{1}=\left\{\begin{array}{l}
2 \varrho \mathbf{A A}, \mathbf{B B} \\
\frac{2 \varrho}{3} \mathbf{A B}, \mathbf{B A}
\end{array},\right. \\
\left(\begin{array}{c}
F_{1}(U) \\
F_{2}(U)
\end{array}\right) & \sim\left(\begin{array}{c}
1 \\
c_{2}
\end{array}\right) \quad \text { with } \quad c_{2}=\left\{\begin{array}{c}
\frac{3}{2 \varrho} \mathbf{A} \mathbf{A}, \mathbf{B B} \\
\frac{1}{2 \varrho} \mathbf{A B}, \mathbf{B A}
\end{array},\right.
\end{aligned}
$$

up to overall normalisation. The complex structure modulus $\varrho=\sqrt{3} R_{2} / 2 R_{1}$ parameterises the ratio of radii $R_{1}, R_{2}$ along the real and imaginary direction on $T_{3}^{2}$, and the constants $c_{i}$ are given in dependence of the lattice orientations on $T_{1}^{2} \times T_{2}^{2}$.

The RR tadpole cancellation conditions on exceptional cycles have no contribution from O6-planes, $\left(L_{3} \ldots L_{6}\right)=(0 \ldots 0)$, and fractional cycles are supersymmetric if they are

\footnotetext{
${ }^{3}$ We are dealing with factorisable tori only, for orientifold models on non-factorisable tori see $23,24,17$.

${ }^{4}$ For the sake of brevity we have set $\left(\tilde{a}_{1}, \tilde{a}_{2}, \tilde{a}_{3}, \tilde{a}_{4}\right) \equiv(P, Q, U, V)$ in the notation of [11.
} 


\begin{tabular}{|c|c|c|c|}
\hline \multirow{2}{*}{ lattice } & $X_{1}$ & $Y_{1}$ & $L_{1}$ \\
& $X_{2}$ & $Y_{2}$ & $L_{2}$ \\
\hline \multirow{2}{*}{$\mathbf{A A}$} & $2 \tilde{a}_{1}+\tilde{a}_{2}$ & $2 \tilde{a}_{3}+\tilde{a}_{4}+b\left(2 \tilde{a}_{1}+\tilde{a}_{2}\right)$ & 8 \\
& $-\left(\tilde{a}_{4}+b \tilde{a}_{2}\right)$ & $\tilde{a}_{2}$ & $8(1-b)$ \\
\hline $\mathbf{A B}$ & $\tilde{a}_{1}+\tilde{a}_{2}$ & $\tilde{a}_{3}+\tilde{a}_{4}+b\left(\tilde{a}_{1}+\tilde{a}_{2}\right)$ & 8 \\
$\mathbf{B A}$ & $\tilde{a}_{3}-\tilde{a}_{b}+b\left(\tilde{a}_{1}-\tilde{a}_{2}\right)$ & $\tilde{a}_{2}-\tilde{a}_{1}$ & $k 8(1-b)$ \\
\hline \multirow{2}{*}{$\mathbf{B B}$} & $\tilde{a}_{1}+2 \tilde{a}_{2}$ & $\tilde{a}_{3}+2 \tilde{a}_{4}+b\left(\tilde{a}_{1}+2 \tilde{a}_{2}\right)$ & 24 \\
& $\tilde{a}_{3}+b \tilde{a}_{4}$ & $-\tilde{a}_{1}$ & $8(1-b)$ \\
\hline
\end{tabular}

Table 1: Bulk coefficients of RR tadpole cancellation and supersymmetry conditions. For shortness, we have set $k=3$ for the $\mathbf{A B}$ lattice on $T_{1}^{2} \times T_{2}^{2}$ and $k=1$ for $\mathbf{B A} . b=0,1 / 2$ parameterises the $\mathbf{a}$ and $\mathbf{b}$ shape on $T_{3}^{2}$.

composed of supersymmetric toroidal cycles and only those exceptional cycles which wrap the four $\mathbb{Z}_{2}$ fixed points on $T_{1}^{2} \times T_{3}^{2}$ traversed by the toroidal cycle with three independent signs of the four potential contributions corresponding to a $\mathbb{Z}_{2}$ eigenvalue and two discrete Wilson lines on $T_{1}^{2} \times T_{3}^{2}$. More details are given in appendix $\mathrm{D}$ and [11].

It turns out that the solutions to the bulk RR tadpole and supersymmetry conditions are independent of the choice of the $T_{1}^{2}$ lattice as we prove in appendix $\mathrm{D}$.

\section{Complete massless spectrum}

In this section, we review the computation of the complete massless, i.e. chiral and nonchiral, open string spectrum in terms of three-cycle intersection numbers and compare the results with those arising from the computation of Chan-Paton matrices. The latter method confirms the formulae inferred in [11].

In the present discussion, special emphasis is placed on states living on branes which are parallel along some two-torus $T_{m}^{2}$ of any $T^{6} / \mathbb{Z}_{2 N}$ background. The generic formulae listed in 111 for those cases are found to be correct except for the case in equation (3.7) where orientifold image branes $a$ and $\left(\theta^{k} a^{\prime}\right)$ are parallel to each other for some $k \in\{0, \ldots, N-1\}$ but orthogonal to the $\Omega \mathcal{R} \theta^{-k}$ and $\Omega \mathcal{R} \theta^{-k+N}$ invariant O6-planes on the two-torus where the $\mathbb{Z}_{2}$ action is trivial. There is furthermore a so far unobserved sign subtlety in (3.12) for brane $a$ on top of $\left(\theta^{k} a^{\prime}\right)$ but displaced from the origin on $T_{3}^{2}$ in case of a Wilson line.

For concreteness, the notation is adapted to the $T^{6} / \mathbb{Z}_{6}^{\prime}$ background with $\vec{v}=$ $(1 / 6,1 / 3,-1 / 2)$, the $\mathbb{Z}_{2}$ invariant two-torus being $T_{2}^{2}$, but the line of reasoning is valid for any other $T^{6} / \mathbb{Z}_{2 N}$ orbifold upon suitable permutation of two-tori. The complete matter spectrum will be needed in order to determine the one-loop running of gauge couplings in section 4 . One should, however, keep in mind that $\mathcal{N}=2$ supersymmetric sectors might become massive, for example by a parallel displacement of branes according to equation (3.3).

For completeness, we also give the closed string spectrum for the $T^{6} / \mathbb{Z}_{6}^{\prime}$ orbifold. 


\subsection{Closed string spectrum}

The untwisted sector contains the supergravity and universal dilaton-axion multiplet. The numbers $n_{C}$ and $n_{V}$ of non-universal chiral and vector multiplets depends on the background. For the $T^{6} / \mathbb{Z}_{6}^{\prime}$ orbifold on $\mathbf{A A}$ or $\mathbf{B A}$ type lattices on $T_{1}^{2} \times T_{2}^{2}$, we obtained $\left(n_{C}, n_{V}\right)=(38-6 b, 8+6 b)$ in 11] and for the $\mathbf{A B}$ and $\mathbf{B B}$ orientations $\left(n_{C}, n_{V}\right)=(46-10 b, 10 b)$ where $b=0, \frac{1}{2}$ parameterises the $\mathbf{a}$ or $\mathbf{b}$ type shape of $T_{3}^{2}$, respectively. Vectors arise always from RR states whereas scalars stem from both NS-NS and RR states. The closed string spectrum preserves $\mathcal{N}=1$ supersymmetry with the fermionic superpartners arising from R-NS and NS-R sectors.

In the Green Schwarz mechanism, an open string U(1) gauge field wrapped around the three-cycle $\Pi$ with orientifold image cycle $\Pi^{\prime}$ becomes massive by absorbing one linear combination of RR scalars dual to the two-form $\int_{\Pi-\Pi^{\prime}} C_{5}$ where $C_{5}$ is the ten dimensional RR 5-form. Due to supersymmetry, at the same time the NS-NS state parameterising a linear combination of complex structure moduli and pertaining to the same chiral multiplet is frozen. Since these closed string sector states carry no gauge representation, the Green Schwarz mechanism in intersecting D-branes on orbifolds does not induce any Fayet-Iliopoulos term provided the three-cycles wrapped by the branes fulfil the three-cycle calibration condition with toroidal part given in equation (2.5), for more details see e.g. the discussion in 25, 26]. This is in contrast to heterotic orbifolds where the anomalous $\mathrm{U}(1)$ factor has a non-vanishing Fayet-Iliopoulos term and a charged scalar forms the transverse degrees of freedom of the massive vector boson.

\subsection{Massless open spectrum from three-cycle intersection numbers}

\subsubsection{For branes at three non-vanishing angles}

In [11], we showed that the intersection number or net chirality $\chi^{a b} \equiv \Pi_{a}^{\text {frac }} \circ \Pi_{b}^{\text {frac }}$ between fractional three-cycles $\Pi_{a}^{\text {frac }}$ and $\Pi_{b}^{\text {frac }}$ and the resulting total amount $\varphi^{a b}$ of bifundamental matter in the $\left(\mathbf{N}_{a}, \overline{\mathbf{N}}_{b}\right)$ representation can be expressed in terms of a sum of toroidal and $\mathbb{Z}_{2}$ invariant intersection numbers,

$$
\begin{aligned}
& I_{a b} \equiv \prod_{i=1}^{3} I_{a b}^{(i)}=\prod_{i=1}^{3}\left(n_{i}^{a} m_{i}^{b}-m_{i}^{a} n_{i}^{b}\right), \\
& I_{a b}^{\mathbb{Z}_{2}} \equiv \sum_{x_{a}^{k} x_{b}^{k}}(-1)^{\tau_{x_{a}^{1}}^{1} x_{a}^{3}+\tau_{x_{b}^{1} x_{b}^{3}}} \delta_{x_{a}^{1} x_{b}^{1}} \delta_{x_{a}^{3} x_{b}^{3}} I_{a b}^{(2)},
\end{aligned}
$$

among brane $a$ and all orbifold images $\left(\theta^{k} b\right)$ for $k=0 \ldots N-1$ on $T^{6} / \mathbb{Z}_{2 N}$,

$$
\begin{gathered}
\chi^{a b} \equiv \chi_{L}^{a b}-\chi_{R}^{a b}=-\sum_{k=0}^{N-1} \frac{I_{a\left(\theta^{k} b\right)}+I_{a\left(\theta^{k} b\right)}^{\mathbb{Z}_{2}}}{2}, \\
\varphi^{a b} \equiv \chi_{L}^{a b}+\chi_{R}^{a b}=\sum_{k=0}^{N-1}\left|\frac{I_{a\left(\theta^{k} b\right)}+I_{a\left(\theta^{k} b\right)}^{\mathbb{Z}_{2}}}{2}\right|,
\end{gathered}
$$

the formula for the chiral plus non-chiral bifundamental matter states $\varphi^{a b}$ being valid only in case of three non-vanishing angles. Here, $x_{a}^{i}$ labels the $\mathbb{Z}_{2}$ fixed points traversed by the 


\begin{tabular}{|c|c|}
\hline \multicolumn{2}{|c|}{ Chiral and non-chiral massless matter on $T^{6} /\left(\mathbb{Z}_{2 N} \times \Omega \mathcal{R}\right)$} \\
\hline rep. & total number $\varphi$ \\
\hline$\left(\mathbf{A d j}_{a}\right)$ & $1+\frac{1}{4} \sum_{k=1}^{N-1}\left|I_{a\left(\theta^{k} a\right)}+I_{a\left(\theta^{k} a\right)}^{\mathbb{Z}_{2}}\right|$ \\
$\left(\mathbf{A n t i}_{a}\right)$ & $\frac{1}{4} \sum_{k=0}^{N-1}\left|I_{a\left(\theta^{k} a^{\prime}\right)}+I_{a\left(\theta^{k} a^{\prime}\right)}^{\mathbb{Z}_{2}}+I_{a}^{\Omega \mathcal{R} \theta^{-k}}+I_{a}^{\Omega \mathcal{R} \theta^{-k+N}}\right|$ \\
$\left(\mathbf{S y m}_{a}\right)$ & $\frac{1}{4} \sum_{k=0}^{N-1}\left|I_{a\left(\theta^{k} a^{\prime}\right)}+I_{a\left(\theta^{k} a^{\prime}\right)}^{\mathbb{Z}_{2}}-I_{a}^{\Omega \mathcal{R} \theta^{-k}}-I_{a}^{\Omega \mathcal{R} \theta^{-k+N}}\right|$ \\
$\left(\mathbf{N}_{a}, \overline{\mathbf{N}}_{b}\right)$ & $\frac{1}{2} \sum_{k=0}^{N-1}\left|I_{a\left(\theta^{k} b\right)}+I_{a\left(\theta^{k} b\right)}^{\mathbb{Z}_{2}}\right|$ \\
$\left(\mathbf{N}_{a}, \mathbf{N}_{b}\right)$ & $\frac{1}{2} \sum_{k=0}^{N-1}\left|I_{a\left(\theta^{k} b^{\prime}\right)}+I_{a\left(\theta^{k} b^{\prime}\right)}^{\mathbb{Z}_{2}}\right|$ \\
\hline
\end{tabular}

Table 2: Chiral plus non-chiral matter states $\varphi$ in $T^{6} /\left(\mathbb{Z}_{2 N} \times \Omega \mathcal{R}\right)$ models for generic non-vanishing angles. For vanishing angles, the formulae are modified as discussed in sections 3.2 .2 to 3.2.4.

toroidal cycle with wrapping number $\left(n_{i}^{a}, m_{i}^{a}\right)$ along $T_{i}^{2}$ and $\tau_{x_{a}^{1} x_{a}^{3}} \in\{0,1\}$ are combinations of Wilson lines and a $\mathbb{Z}_{2}$ eigenvalue. The complete matter spectrum for this case is given in table 2 .

Supersymmetric brane configurations are also possible with either one or three vanishing angles. The net-chirality $\chi^{a b}$ is still computed by means of three-cycle intersection numbers (3.1), but a zero can either correspond to no massless state or $\mathcal{N}=2$ supersymmetric non-chiral matter pairs. If the branes are parallel along the $\mathbb{Z}_{2}$ invariant two-torus $T_{2}^{2}$ (and also parallel to the relevant O6-planes for $b=\left(\theta^{k} a^{\prime}\right)$ ), the number of non-chiral pairs is simply computed from the intersection numbers on $T_{1}^{2} \times T_{3}^{2}$ in a six-dimensional set-up. In [11, we were only able to list the remaining cases with branes parallel along $T_{1}^{2}$ or $T_{3}^{2}$ by silently employing the method of Chan-Paton matrices illustrated in detail in section 3.3. Furthermore, we did not consider before the case with brane $a$ and its orientifold image $\left(\theta^{k} a^{\prime}\right)$ parallel along $T_{2}^{2}$ but orthogonal to the $\Omega \mathcal{R} \theta^{-k}$ and $\Omega \mathcal{R} \theta^{-k+N}$ invariant O6-planes given below in (3.7). There is also a sign subtlety for branes parallel on, e.g., $T_{3}^{2}$ when carrying a Wilson line and displaced from the origin on the same two-torus, see equation (3.12).

The formulae for branes parallel along some two-torus are modified as follows, with a detailed discussion of the derivation to follow below in section 3.3:

\subsubsection{For branes parallel along all three tori}

- The $a a$ sector provides a vector multiplet of $\mathrm{U}\left(N_{a}\right)$ and one chiral multiplet in the adjoint representation.

- Branes $a_{1}, a_{2}$ with identical position, opposite $\mathbb{Z}_{2}$ eigenvalues and no relative Wilson line, i.e. $\tau_{a_{1}}^{0}=\tau_{a_{2}}^{0}+1 \bmod 2$ and $\tau_{a_{1}}^{i}=\tau_{a_{2}}^{i}(i=1,3)$, contribute $2 \times\left[\left(\mathbf{N}^{1}{ }_{a}, \overline{\mathbf{N}^{2}}{ }_{a}\right)+\right.$ c.c. $]$, or in the special case of orientifold image branes, i.e. $a_{2}=\left(\theta^{k} a_{1}^{\prime}\right)$ for some $k \in$ $\{0, \ldots, N-1\}$, the bifundamental representations are replaced by $2 \times\left[\mathbf{A n t i}_{a}+\right.$ c.c. $]$. 
- Branes $a$ and $b$ parallel on $T_{i}^{2}$ but either spatially separated, $\Delta \sigma_{a b}^{i} \neq 0(i=1,3),{ }^{5}$ or with a relative Wilson line, $\Delta \tau_{a b}^{i} \neq 0(i=1,3)$, do not contribute to the massless spectrum due to a shift in the mass formula for a state with given momentum and winding numbers $p_{i}, q_{i} \in \mathbb{Z}$ along $T_{i}^{2}$,

$$
(\operatorname{mass})^{2} \sim\left(\left(\frac{1}{r_{\|}^{(i)}}\right)^{2}\left(p_{i}+\frac{\Delta \tau^{i}}{2}\right)^{2}+\left(\frac{r_{\perp}^{(i)}}{\alpha^{\prime}}\right)^{2}\left(q_{i}+\Delta \sigma^{i}\right)^{2}\right)
$$

where $r_{\|}^{(i)}$ is the length of the one-cycle on $T_{i}^{2}$ and $r_{\perp}^{(i)}$ the distance of two copies of the same one-cycle on $T_{i}^{2}$, both in appropriate units, and the relative Wilson lines and spatial displacements are parameterised by $\Delta \tau^{i} \in\{0,1\}$ and $\Delta \sigma^{i} \in\{0,1 / 2\}$ for $i=1,3$, whereas $\Delta \tau^{2}$ and $\Delta \sigma^{2}$ can vary continuously.

\subsubsection{For branes parallel along the $\mathbb{Z}_{2}$ invariant $T_{2}^{2}$}

- The bifundamental states for $a$ and $\left(\theta^{k} b\right)$ parallel on $T_{2}^{2}$ for a given $k$ and at angles on $T_{1}^{2} \times T_{3}^{2}$ are grouped in $\mathcal{N}=2$ supersymmetric non-chiral pairs counted by twice the intersection number on $T_{1}^{2} \times T_{3}^{2}$. The corresponding term for the given $k$ in table 2 is replaced by

$$
\begin{aligned}
\varphi^{a b, \| T_{2}^{2}} & \rightarrow\left|I_{a\left(\theta^{k} b\right)}^{(1 \cdot 3)}+I_{a\left(\theta^{k} b\right)}^{\mathbb{Z}_{2},(1 \cdot 3)}\right|, \\
\varphi^{\mathbf{A d j}_{a}, \| T_{2}^{2}} & \rightarrow \frac{1}{2}\left|I_{a\left(\theta^{k} a\right)}^{(1 \cdot 3)}+I_{a\left(\theta^{k} a\right)}^{\mathbb{Z}_{2},(1 \cdot 3)}\right|,
\end{aligned}
$$

where the upper index $(1 \cdot 3)$ indicates that intersection numbers are computed only on $T_{1}^{2} \times T_{3}^{2}$, e.g. $I_{a b}^{(1 \cdot 3)}=\prod_{i=1,3}\left(n_{i}^{a} m_{i}^{b}-m_{i}^{a} n_{i}^{b}\right)$. This case applies e.g. to the adjoint representations in a $T^{6} / \mathbb{Z}_{4}$ background and is found to agree with the $\left(\boldsymbol{6}_{i}, \boldsymbol{6}_{i+2}\right)$ spectra of [27, 28]. The modification for $a$ parallel to some orientifold image brane $\left(\theta^{k} b^{\prime}\right)$ is obvious.

- If brane $a$ and its orientifold image $\left(\theta^{k} a^{\prime}\right)$ are parallel to the $\Omega \mathcal{R} \theta^{-k}$ and $\Omega \mathcal{R} \theta^{-k+N}$ invariant O6-planes on $T_{2}^{2}$ for some $k$, the corresponding sector stays $\mathcal{N}=2$ supersymmetric, and the matter states for the given $k$ are counted by

$$
\left.\begin{array}{l}
\varphi^{\mathbf{A n t i}_{a},\left\|T_{2}^{2}\right\| \Omega \mathcal{R} \theta^{-k(+N)}} \\
\varphi^{\mathbf{S y m}_{a},\left\|T_{2}^{2}\right\| \Omega \mathcal{R} \theta^{-k(+N)}}
\end{array}\right\} \rightarrow \frac{1}{2}\left|I_{a\left(\theta^{k} a^{\prime}\right)}^{(1 \cdot 3)}+I_{a\left(\theta^{k} a^{\prime}\right)}^{\mathbb{Z}_{2},(1 \cdot 3)} \pm\left(I_{a}^{\Omega \mathcal{R} \theta^{-k},(1 \cdot 3)}+I_{a}^{\Omega \mathcal{R} \theta^{-k+N},(1 \cdot 3)}\right)\right|
$$

the upper sign being valid for antisymmetric representations and the lower one for symmetric ones.

- If brane $a$ and its orientifold image $\left(\theta^{k} a^{\prime}\right)$ are parallel among themselves but orthogonal to the $\Omega \mathcal{R} \theta^{-k}$ and $\Omega \mathcal{R} \theta^{-k+N}$ invariant O6-planes on $T_{2}^{2}$ for some given $k$, the O6-planes break $\mathcal{N}=2$ down to $\mathcal{N}=1$ supersymmetry, as can be seen also from

\footnotetext{
${ }^{5}$ In slight abuse of notation, displacements from the origin along one-cycles $\pi_{k}$ are labelled by $\sigma_{k} \in$ $\{0,1 / 2\}$, but $\Delta \sigma^{i}$ denotes a relative distance of branes along $T_{i}^{2}$.
} 
the fact that the net-chirality of symmetric and antisymmetric states is in general opposite and non-vanishing for the given $k$,

$$
\left.\begin{array}{l}
\chi^{\mathbf{A n t i}_{a}, \| T_{2}^{2} \perp \Omega \mathcal{R} \theta^{-k(+N)}} \\
\chi^{\mathbf{S y m}_{a}, \| T_{2}^{2} \perp \Omega \mathcal{R} \theta^{-k(N)}}
\end{array}\right\} \rightarrow \mp \frac{1}{4}\left(I_{a}^{\Omega \mathcal{R} \theta^{-k}}+I_{a}^{\Omega \mathcal{R} \theta^{-k+N}}\right) .
$$

There is an equal amount of antisymmetric and symmetric representations (up to complex conjugation) for the given $k$,

$$
\left.\begin{array}{l}
\varphi^{\mathbf{A n t i}_{a}, \| T_{2}^{2} \perp \Omega \mathcal{R} \theta^{-k(+N)}} \\
\varphi^{\mathbf{S y m}_{a}, \| T_{2}^{2} \perp \Omega \mathcal{R} \theta^{-k(+N)}}
\end{array}\right\} \rightarrow \frac{1}{2}\left|I_{a\left(\theta^{k} a^{\prime}\right)}^{(1 \cdot 3)}+I_{a\left(\theta^{k} a^{\prime}\right)}^{\mathbb{Z}_{2},(1 \cdot 3)}\right| .
$$

This case had not been discussed in [11] and is presented here for the first time.

\subsubsection{For branes parallel along $T_{3}^{2}$ where $\mathbb{Z}_{2}$ acts non-trivially}

The case with branes parallel along $T_{1}^{2}$ arises by permutation of indices and is not listed here in detail.

- The sector of branes $a$ and $\left(\theta^{k} b\right)$ parallel to each other on $T_{3}^{2}$ for some $k$ is in general $\mathcal{N}=1$ supersymmetric with net-chirality

$$
\chi^{a b, \| T_{3}^{2}} \rightarrow-\frac{1}{2} I_{a\left(\theta^{k} b\right)}^{\mathbb{Z}_{2}},
$$

which turns out to be zero for a non-vanishing relative distance or Wilson line,

$$
\left(\Delta \sigma_{a\left(\theta^{k} b\right)}^{3}, \Delta \tau_{a\left(\theta^{k} b\right)}^{3}\right) \neq(0,0)
$$

The total counting of massless bifundamental states for branes $a$ and $\left(\theta^{k} b\right)$ on top of each other on $T_{3}^{2}$ is

$$
\begin{aligned}
\varphi^{a b, \| T_{3}^{2}} & \rightarrow\left\{\begin{array}{cc}
\left|I_{a\left(\theta^{k} b\right)}^{(1 \cdot 2)}\right| & \left(\Delta \sigma_{a\left(\theta^{k} b\right)}^{3}, \Delta \tau_{a\left(\theta^{k} b\right)}^{3}\right)=(0,0) \\
0 & \left(\Delta \sigma_{a\left(\theta^{k} b\right)}^{3}, \Delta \tau_{a\left(\theta^{k} b\right)}^{3}\right) \neq(0,0)
\end{array},\right. \\
\varphi^{\mathbf{A d j}_{a}, \| T_{3}^{2}} & \rightarrow \frac{1}{2}\left|I_{a\left(\theta^{k} a\right)}^{(1 \cdot 2)}\right| \quad \text { for } \quad k \neq 0 .
\end{aligned}
$$

This formula in particular applies to the counting of adjoint representation in a $T^{6} / \mathbb{Z}_{6}^{\prime}$ background where $\varphi^{\mathbf{A d j}}=1+\prod_{i=1}^{2}\left|n_{i}^{2}+n_{i} m_{i}+m_{i}^{2}\right|$.

- The O6-planes invariant under $\Omega \mathcal{R} \theta^{-k}$ and $\Omega \mathcal{R} \theta^{-k+N}$ are orthogonal to each other on $T_{3}^{2}$, and branes $a$ lie on one of these if they are on top of $\left(\theta^{k} a^{\prime}\right)$ for some $k$. The sector is $\mathcal{N}=1$ supersymmetric with net-chirality

$$
\begin{aligned}
& \left.\left.\chi^{\chi_{\mathbf{S y m}_{a}, \| T_{3}^{2}}^{\mathbf{A n t i}_{a}, \| T_{3}^{2}}}\right\}\right\} \rightarrow-\frac{1}{4}\left(I_{a\left(\theta^{k} a^{\prime}\right)}^{\mathbb{Z}_{2}} \pm I_{a}^{\Omega \mathcal{R} \theta^{-x}}\right) \\
& \text { with the exponent } \quad x=\left\{\begin{array}{cc}
k & a \perp \Omega \mathcal{R} \theta^{-k} \text { on } T_{3}^{2} \\
k+N & a \perp \Omega \mathcal{R} \theta^{-k+N} \text { on } T_{3}^{2}
\end{array} .\right.
\end{aligned}
$$


The total number of antisymmetric and symmetric states is counted by

$$
\begin{aligned}
& \left.\begin{array}{l}
\varphi^{\mathbf{A n t i}} \mathbf{A n}_{a}, \| T_{3}^{2} \\
\varphi^{\mathbf{S y m}_{a}, \| T_{3}^{2}}
\end{array}\right\} \rightarrow \frac{1}{2}\left|I_{a\left(\theta^{k} a^{\prime}\right)}^{(1 \cdot 2)} \pm \tilde{c} I_{a}^{\Omega \mathcal{R} \theta^{-y}}\right| \\
& \text { with } y=\left\{\begin{array}{ccc}
k & a \| \Omega \mathcal{R} \theta^{-k} \text { on } T_{3}^{2} \\
k+N & a \| \Omega \mathcal{R} \theta^{-k+N} \text { on } T_{3}^{2}
\end{array} .\right.
\end{aligned}
$$

The constant $\tilde{c}=e^{2 \pi i \tau^{3} \Delta \sigma^{3}}$ takes into account that for branes which are displaced from the origin on $T_{3}^{2}$, the $\mathbb{Z}_{2}$ invariant intersection points are exchanged under the orientifold projection, and a sign factor arises if the brane carries a discrete Wilson line on $T_{3}^{2}$. This permutation of $\mathbb{Z}_{2}$ fixed points only occurs on tilted tori such as the b type torus on $T_{3}^{2}$ or the $\mathrm{SU}(3)$ invariant ones $\mathbf{A}$ and $\mathbf{B}$ on $T_{1}^{2}$, but not on the $\mathbf{a}$ type $T_{3}^{2}$.

The sign factor $\tilde{c}$ had not been observed in [11], but is necessary in section 7 in order to avoid a mismatch in the chiral and non-chiral counting of antisymmetric states, $\left|\chi^{\mathbf{A n t i}_{b}, \| T_{1}^{2}}\right| \leq \varphi^{\mathbf{A n t i}_{b}, \| T_{1}^{2}}$, on stack $b$ defined in tables 14 and 15 .

In the following section we verify explicitly the correctness of these formulae (in the absence of Wilson lines) using Chan-Paton labels. The antisymmetric and symmetric representations on orientifold invariant D6-branes with $\mathrm{SO}(2 N)$ or $\mathrm{Sp}(2 N)$ gauge groups require a different treatment displayed in appendix $\mathrm{Q}$.

\subsection{Massless spectrum from Chan-Paton matrices}

The $\gamma$-matrices associated to the $\mathbb{Z}_{2} \equiv \theta^{N}$ and $\Omega \mathcal{R} \theta^{-k}$ action on the Chan-Paton matrices $\lambda_{a\left(\theta^{k} b\right)}$ and $\lambda_{a\left(\theta^{k} a^{\prime}\right)}$ in a $T^{6} / \mathbb{Z}_{2 N}$ background can be chosen in agreement with 29], where bulk D7-branes on $T^{4} / \mathbb{Z}_{2}$ had been analysed,

$$
\begin{aligned}
& \gamma_{\mathbb{Z}_{2}}=\left(\begin{array}{cc}
\mathbb{I} & 0 \\
0 & -\mathbb{I}
\end{array}\right), \quad \gamma_{\Omega \mathcal{R} \theta^{-k}}=\left(\begin{array}{cc}
0 & \mathbf{I} \\
\mathbb{I} & 0
\end{array}\right), \\
& \gamma_{\Omega \mathcal{R} \theta^{-k+N}} \equiv \gamma_{\Omega \mathcal{R} \theta^{-k}} \cdot \gamma_{\mathbb{Z}_{2}}=\left(\begin{array}{cc}
0 & -\mathbf{I} \\
\mathbf{I} & 0
\end{array}\right) .
\end{aligned}
$$

The gauge group for a $2 N_{a} \times 2 N_{a}$ matrix $\lambda_{a a}$ derived in [29] is $\mathrm{U}\left(N_{a}\right) \times \mathrm{U}\left(N_{a}\right)$ for a generic brane $a \neq\left(\theta^{k} a^{\prime}\right)$ not on top of the O7-planes and $\mathrm{U}\left(N_{a}\right)$ if $a=\left(\theta^{k} a^{\prime}\right)$ for some $k$. This set-up can be viewed as the special case of fractional D7-branes $a_{i}(i=1,2)$ and their orbifold images $\left(\theta^{k} a_{i}\right)$ wrapping fractional cycles $\Pi_{a_{i}}^{\text {frac }}$ such that

$$
\Pi_{a}^{\mathrm{bulk}}=\Pi_{a_{1}}^{\mathrm{frac}}+\Pi_{a_{2}}^{\mathrm{frac}} \quad \text { and } \quad N_{a}=N_{a}^{1}=N_{a}^{2} .
$$

Allowing for different stack sizes $N_{a}^{i}, N_{b}^{j} \geq 0$ for $i, j=1,2$, the Chan-Paton labels decompose as

$$
\begin{aligned}
& \lambda_{a\left(\theta^{k} b\right)} \simeq\left(\begin{array}{cc}
\left(\mathbf{N}_{a}^{1}, \overline{\mathbf{N}}_{b}\right) & \left(\mathbf{N}_{a}^{1}, \overline{\mathbf{N}}_{b}\right) \\
\left(\mathbf{N}_{a}^{2}, \overline{\mathbf{N}}_{b}\right) & \left(\mathbf{N}_{a}^{2}, \overline{\mathbf{N}}_{b}\right)
\end{array}\right), \\
& \lambda_{a\left(\theta^{k} a^{\prime}\right)} \simeq\left(\begin{array}{cc}
\left(\mathbf{N}_{a}^{1}, \mathbf{N}_{a}^{2}\right) & \mathbf{A n t i}_{a}^{\mathbf{1}}+\mathbf{S y m}_{a}^{\mathbf{1}} \\
\mathbf{A n t i}_{a}^{2}+\mathbf{S y m}_{a}^{2} & \left(\mathbf{N}_{a}^{2}, \mathbf{N}_{a}^{1}\right)
\end{array}\right)
\end{aligned}
$$


where for the $a\left(\theta^{k} a^{\prime}\right)$ states it was used that the orientifold projection exchanges $\mathbb{Z}_{2}$ eigenvalues $\tau_{a}^{0} \rightarrow \tau_{a^{\prime}}^{0}=\tau_{a}^{0}+1$ and acts as complex conjugation on the representations, i.e. $\mathbf{N}^{1}{ }_{a^{\prime}}=\overline{\mathbf{N}}_{a}$ and $\mathbf{N}_{a^{\prime}}{ }^{a}=\overline{\mathbf{N}}_{a}$.

The line of argument carries directly over to fractional D6-branes and O6-planes in a $T^{6} / \mathbb{Z}_{2 N}$ background with a single $\mathbb{Z}_{2}$ sub-symmetry. The cases with $T^{6} / \mathbb{Z}_{2 N} \times \mathbb{Z}_{2 M}$ backgrounds require a second $\gamma_{\mathbb{Z}_{2}}$ matrix for the other $\mathbb{Z}_{2}$ sub-symmetry and will be discussed elsewhere [30].

In order to determine the massless spectrum, one further needs to know the invariance properties of the brane intersections in order to determine which projection condition on the Chan-Paton label applies, and finally the number of massless states, their chiralities and the associated eigenvalues $\left(a_{\mathbb{Z}_{2}}, a_{\Omega \mathcal{R} \theta^{-k}}, a_{\Omega \mathcal{R} \theta^{-k+N}}\right)$ under the orbifold and orientifold projections with $a_{\Omega \mathcal{R} \theta^{-k+N}}=a_{\mathbb{Z}_{2}} \cdot a_{\Omega \mathcal{R} \theta^{-k}}$ for consistency.

Let us start by listing the massless multiplets for branes at relative angles $\pi\left(\phi_{1}, \phi_{2}, \phi_{3}\right)$ which comply with the supersymmetry constraint $\sum_{i=1}^{3} \phi_{i}=0 \bmod 2$ :

- On parallel branes with $\pi\left(\phi_{1}, \phi_{2}, \phi_{3}\right)=(0,0,0)$ there exist a $\mathbb{Z}_{2}$ even vector and chiral multiplet plus two further $\mathbb{Z}_{2}$ odd chiral multiplets.

- At intersections of branes $a$ and $\left(\theta^{k} b\right)$ with all $\phi_{i} \neq 0$, the $a\left(\theta^{k} b\right)$ sector provides one bosonic and fermionic degree of freedom. Together with the $\left(\theta^{k} b\right) a$ sector, these group into a $\mathbb{Z}_{2}$ even chiral multiplet.

- Branes parallel along the $\mathbb{Z}_{2}$ invariant two-torus $T_{2}^{2}$ at angles $\pi(\phi, 0,-\phi)$ provide two $\mathbb{Z}_{2}$ odd multiplets of opposite chirality.

- Branes parallel along a two-torus where $\mathbb{Z}_{2}$ acts non-trivially, i.e. $\pi(\phi,-\phi, 0)$ or $\pi(0, \phi,-\phi)$, provide one $\mathbb{Z}_{2}$ even and one $\mathbb{Z}_{2}$ odd multiplet with opposite chiralities.

More details on the NS and R states are given in appendix B, see in particular table 24.

The chirality of the massless multiplets in each sector can in principle directly be read off from the massless $\mathrm{R}$ state in table 24. We will in the following, however, ignore relative chiralities among different sectors since they are easily recovered from the threecycle intersection numbers in equations (3.1), (3.7), (3.8) and (3.11). Opposite chiralities within a given sector are on the other hand taken into account.

\subsubsection{Branes parallel on all tori}

The case for parallel branes has been discussed in detail in section 3.2.2. In the absence of Wilson lines, the representations pertaining to $\mathbb{Z}_{2}$ even states are read off from the diagonal entries in $\lambda_{a\left(\theta^{k} b\right)}$, those of the $\mathbb{Z}_{2}$ odd states from the off-diagonals in (3.15).

\subsubsection{Branes at non-trivial angles}

The matter states on branes with non-vanishing intersection numbers on all tori can be computed wholly from the intersection numbers. We verify here that using the method of Chan-Paton labels, one recovers the spectrum in table 2. The present computation carries over to the case with a vanishing angle as discussed below in sections 3.3 .3 and 3.3.4. 
For two branes $a$ and $\left(\theta^{k} b\right)$ at non-trivial angles, the intersections can be either $\mathbb{Z}_{2}$ invariant with their abundance counted by $x_{\mathbb{Z}_{2}} \equiv\left|I_{a\left(\theta^{k} b\right)}^{\mathbb{Z}_{2}}\right|$ or form pairs under $\mathbb{Z}_{2}$ which are counted by

$$
x_{\mathbb{Z}_{2}-\text { pairs }} \equiv \frac{\left|I_{a\left(\theta^{k} b\right)}\right|-\left|I_{a\left(\theta^{k} b\right)}^{\mathbb{Z}_{2}}\right|}{2} .
$$

At this point, we focus on branes without relative Wilson lines, $\tau_{a}^{i}=\tau_{b}^{i}$ for $i=1,3$, but include arbitrary $\mathbb{Z}_{2}$ eigenvalues $\tau_{a}^{0}, \tau_{b}^{0} \in\{0,1\}$. We will briefly comment on the case with relative Wilson lines below. The following representations occur:

- At intersection points which are exchanged by the $\mathbb{Z}_{2}$ symmetry, there is no projection on the Chan-Paton matrix. Independently of any relative $\mathbb{Z}_{2}$ eigenvalue $i, j=1,2$, the contribution to the massless spectrum is

$$
x_{\mathbb{Z}_{2}-\text { pairs }} \times\left(\mathbf{N}_{a}^{i}, \overline{\mathbf{N}}_{b}^{j}\right) .
$$

- At $\mathbb{Z}_{2}$ invariant intersections, the projection on the Chan-Paton label leads to

$$
a_{\mathbb{Z}_{2}}=1: x_{\mathbb{Z}_{2}} \times\left(\mathbf{N}_{a}^{i}, \overline{\mathbf{N}}_{b}^{i}\right), \quad \text { or } \quad a_{\mathbb{Z}_{2}}=-1: x_{\mathbb{Z}_{2}} \times\left(\mathbf{N}_{a}^{i}, \overline{\mathbf{N}}_{b}^{j}\right)_{i \neq j}
$$

- Adding up the contributions from $\mathbb{Z}_{2}$ invariant intersection points and pairs which are exchanged while taking care of relative signs among $I_{a\left(\theta^{k} b\right)}$ and $I_{a\left(\theta^{k} b\right)}^{\mathbb{Z}_{2}}$ leads to

$$
\frac{\left|I_{a\left(\theta^{k} b\right)}+I_{a\left(\theta^{k} b\right)}^{\mathbb{Z}_{2}}\right|}{2} \times\left(\mathbf{N}_{a}^{i}, \overline{\mathbf{N}}_{b}^{j}\right) \quad \text { for } \quad i, j=1,2,
$$

which is clearly in agreement with the multiplicity derived from intersection numbers alone in table 2 .

Our derivation of massless matter representations is tailor-made for branes without relative Wilson lines. However, from the construction and by comparison with the result in table 2, one sees clearly that a relative Wilson line will provide a flip of the sign $a_{\mathbb{Z}_{2}}$ in the projection of the Chan-Paton matrix at some of the $\mathbb{Z}_{2}$ invariant intersection points. The counting of massless bifundamental representations (3.19) remains valid, but $x_{\mathbb{Z}_{2}}=\left|I_{a\left(\theta^{k} b\right)}^{\mathbb{Z}_{2}}\right|$ looses the geometric interpretation of counting simply $\mathbb{Z}_{2}$ invariant intersection points. Instead, it counts intersections with relative signs.

For the computation of symmetric and antisymmetric representations the orbits of intersection numbers have to be divided differently into their invariance properties and abundances as follows:

- points fixed under both $\Omega \mathcal{R} \theta^{-k}$ and $\mathbb{Z}_{2}$ :

$$
y_{\mathbb{Z}_{2}+\Omega \mathcal{R} \theta^{-k}} \equiv\left|I_{a}^{\mathbb{Z}_{2}+\Omega \mathcal{R} \theta^{-k}}\right|,
$$

- orbits of points fixed under $\mathbb{Z}_{2}$ but forming pairs under $\Omega \mathcal{R} \theta^{-k}$ :

$$
y_{\mathbb{Z}_{2}} \equiv \frac{\left|I_{a\left(\theta^{k} a^{\prime}\right)}^{\mathbb{Z}_{2}}\right|-\left|I_{a}^{\mathbb{Z}_{2}+\Omega \mathcal{R} \theta^{-k}}\right|}{2},
$$




\begin{tabular}{|c|c|c|}
\hline \multicolumn{3}{|c|}{ Matter at $a\left(\theta^{k} a^{\prime}\right)+\left(\theta^{k} a^{\prime}\right) a$ intersections on $T^{6} / \mathbb{Z}_{2 N}$} \\
\hline mult. & $\left(a_{\mathbb{Z}_{2}}, a_{\Omega \mathcal{R} \theta^{-k}}, a_{\Omega \mathcal{R} \theta^{-k+N}}\right)$ & rep. \\
\hline$y_{0}$ & $(*, *, *)$ & $\mathbf{A n t i}_{a}^{i}+\mathbf{S y m}_{a}^{i}$ \\
\hline$y_{\mathbb{Z}_{2}}$ & $(+, *, *)$ & - \\
& $(-, *, *)$ & $\mathbf{A n t i}_{a}^{i}+\mathbf{S y m}_{a}^{i}$ \\
\hline$y_{\Omega \mathcal{R} \theta^{-k}}$ & $(*,+, *)$ & $\mathbf{S y m}_{a}^{i}$ \\
& $(*,-, *)$ & $\mathbf{A n t i}_{a}^{i}$ \\
\hline$y_{\Omega \mathcal{R} \theta^{-k+N}}$ & $(*, *,+)$ & $\mathbf{A n t i}_{a}^{i}$ \\
& $(*, *,-)$ & $\mathbf{S y m}_{a}^{i}$ \\
\hline$y_{\mathbb{Z}_{2}+\Omega \mathcal{R} \theta^{-k}}$ & $(+, \pm, \pm)$ & - \\
& $(-,+,-)$ & $\mathbf{S y m}_{a}^{i}$ \\
& $(-,-,+)$ & $\mathbf{A n t i}_{a}^{i}$ \\
\hline
\end{tabular}

Table 3: Counting of symmetric and antisymmetric representations at $a\left(\theta^{k} a^{\prime}\right)+\left(\theta^{k} a^{\prime}\right) a$ intersections in a $T^{6} /\left(\mathbb{Z}_{2 N} \times \Omega \mathcal{R}\right)$ background. A star ${ }^{*}$ denotes no projection condition. Only two signs are independent, $a_{\Omega \mathcal{R} \theta^{-k+N}}=a_{\mathbb{Z}_{2}} \cdot a_{\Omega \mathcal{R} \theta^{-k}}$.

- orbits of points fixed under $\Omega \mathcal{R} \theta^{-k}$ or $\Omega \mathcal{R} \theta^{-k+N}$ but forming pairs under $\mathbb{Z}_{2}$ :

$$
\begin{aligned}
y_{\Omega \mathcal{R} \theta^{-k}} & \equiv \frac{\left|I_{a}^{\Omega \mathcal{R} \theta^{-k}}\right|-\left|I_{a}^{\mathbb{Z}_{2}+\Omega \mathcal{R} \theta^{-k}}\right|}{2} \text { and } \\
y_{\Omega \mathcal{R} \theta^{-k+N}} & \equiv \frac{\left|I_{a}^{\Omega \mathcal{R} \theta^{-k+N}}\right|-\left|I_{a}^{\mathbb{Z}_{2}+\Omega \mathcal{R} \theta^{-k}}\right|}{2},
\end{aligned}
$$

- orbits of intersection points which are not fixed under $\mathbb{Z}_{2}, \Omega \mathcal{R} \theta^{-k}$ or $\Omega \mathcal{R} \theta^{-k+N}$ :

$$
y_{0} \equiv \frac{1}{4}\left\{\left|I_{a\left(\theta^{k} a^{\prime}\right)}\right|-\left|I_{a\left(\theta^{k} a^{\prime}\right)}^{\mathbb{Z}_{2}}\right|-\left|I_{a}^{\Omega \mathcal{R} \theta^{-k}}\right|-\left|I_{a}^{\Omega \mathcal{R} \theta^{-k+N}}\right|+2\left|I_{a}^{\mathbb{Z}_{2}+\Omega \mathcal{R} \theta^{-k}}\right|\right\} .
$$

The quantity $\left|I_{a}^{\mathbb{Z}_{2}+\Omega \mathcal{R} \theta^{-k}}\right|$ can be determined on a case-by-case basis for a given brane configuration and $T^{6} / \mathbb{Z}_{2 N}$ background. As a simple check, one can verify that these orbits add up to the total number of intersections,

$$
\left|I_{a\left(\theta^{k} a^{\prime}\right)}\right|=4 y_{0}+2\left(y_{\mathbb{Z}_{2}}+y_{\Omega \mathcal{R} \theta^{-k}}+y_{\Omega \mathcal{R} \theta^{-k+N}}\right)+y_{\mathbb{Z}_{2}+\Omega \mathcal{R} \theta^{-k}} .
$$

We do not give the details here since this number does not appear in the final result of counting chiral and non-chiral matter representations.

Depending on the various signs in the orbifold or orientifold projections, the states are as given in table 3. The formula in table 2 is recovered by adding all matter states up for a given choice of $\left(a_{\mathbb{Z}_{2}}, a_{\Omega \mathcal{R} \theta^{-k}}, a_{\Omega \mathcal{R} \theta^{-k+N}}\right)$.

\subsubsection{Branes parallel along the $\mathbb{Z}_{2}$ invariant torus}

For branes parallel along $T_{m}^{2}$ and at angles on $T_{n}^{2} \times T_{p}^{2}$ with $\{m, n, p\}$ cyclic permutations of $\{1,2,3\}$, a similar counting of orbits of intersection points can be performed 


\begin{tabular}{|c|c|c|}
\hline \multicolumn{3}{|c|}{$\mathcal{N}=2$ Matter for branes $\|$ on $T_{2}^{2}$ and $\| \Omega \mathcal{R} \theta^{-k}$} \\
\hline mult. & $\left(a_{\mathbb{Z}_{2}}, a_{\Omega \mathcal{R} \theta^{-k}}, a_{\Omega \mathcal{R} \theta^{-k+N}}\right)$ & rep. \\
\hline$x_{\mathbb{Z}_{2}-\text { pairs }}^{(2)}$ & $(*, *, *)$ & $\left(\mathbf{N}_{a}^{i}, \overline{\mathbf{N}}_{b}^{j}\right)+$ c.c. \\
\hline$x_{\mathbb{Z}_{2}}^{(2)}$ & $(+, *, *)$ & $\left(\mathbf{N}_{a}^{i}, \overline{\mathbf{N}}_{b}^{i}\right)+$ c.c. \\
& $(-, *, *)$ & $\left(\mathbf{N}_{a}^{i}, \overline{\mathbf{N}}_{b}^{j}\right)_{i \neq j}+$ c.c. \\
\hline$y_{0}^{(2)}$ & $(*, *, *)$ & $\mathbf{A n t i}_{a}^{i}+\mathbf{S y m}_{a}^{i}+$ c.c. \\
\hline$y_{\mathbb{Z}_{2}}^{(2)}$ & $(+, *, *)$ & - \\
& $(-, *, *)$ & $\mathbf{A n t i}_{a}^{i}+\mathbf{S y m}_{a}^{i}+$ c.c. \\
\hline$y_{\Omega \mathcal{R} \theta^{-k}}^{(2)}$ & $(*,+, *)$ & $\mathbf{S y m}_{a}^{i}+$ c.c. \\
& $(*,-, *)$ & $\mathbf{A n t i}_{a}^{i}+$ c.c. \\
\hline$y_{\Omega \mathcal{R} \theta^{-k+N}}^{(2)}$ & $(*, *,+)$ & $\mathbf{A n t i}_{a}^{i}+$ c.c. \\
& $(*, *,-)$ & $\mathbf{S y m}_{a}^{i}+$ c.c. \\
\hline$y_{\mathbb{Z}_{2}+\Omega \mathcal{R} \theta^{-k}}^{(2)}$ & $(+, \pm, \pm)$ & - \\
& $(-,+,-)$ & $\mathbf{S y m}_{a}^{i}+$ c.c. \\
& $(-,-,+)$ & $\mathbf{A n t i}_{a}^{i}+$ c.c. \\
\hline
\end{tabular}

Table 4: $\mathcal{N}=2$ supersymmetric sectors occurring for branes parallel along the $\mathbb{Z}_{2}$ invariant $T_{2}^{2}$. The symmetric and antisymmetric representations only fit into $\mathcal{N}=2$ multiplets if they are also parallel to the $\Omega \mathcal{R} \theta^{-k}$ and $\Omega \mathcal{R} \theta^{-k+N}$ invariant O6-planes. For branes orthogonal to these O6-planes see table 5 .

as in section 3.3.2. In the following, we will label these orbits of points on $T_{n}^{2} \times T_{p}^{2}$ for branes parallel along $T_{m}^{2}$ by an upper index $(m)$. The abundance of $\mathbb{Z}_{2}$ invariant intersection points on $T_{1}^{2} \times T_{3}^{2}$ for branes parallel along $T_{2}^{2}$ is, e.g., denoted by $x_{\mathbb{Z}_{2}}^{(2)}=\left|I_{a b}^{\mathbb{Z}_{2},(1 \cdot 3)}\right|=\left|\sum_{x_{a}^{k} x_{b}^{k}}(-1)^{\tau_{x_{a}^{1} x_{a}^{3}}+\tau_{x_{b}^{1}} x_{b}^{3}} \delta_{x_{a}^{1} x_{b}^{1}} \delta_{x_{a}^{3} x_{b}^{3}}\right|{ }^{6}$

The two massless multiplets for branes parallel along $T_{2}^{2}$ have the same (negative) $\mathbb{Z}_{2}$ eigenvalue as listed in table 24 with opposite chirality thereby forming an $\mathcal{N}=2$ hyper multiplet. This statement remains true for branes $a$ and $\left(\theta^{k} a^{\prime}\right)$ parallel along $T_{2}^{2}$ for some $k$ and also parallel to the $\Omega \mathcal{R} \theta^{-k}$ and $\Omega \mathcal{R} \theta^{-k+N}$ invariant O6-planes there. In these two cases, the spectrum is computed simply from intersection numbers on $T_{1}^{2} \times T_{3}^{2}$ leading to the states listed in table 4 .

The situation changes if brane $a$ and its orientifold image $\left(\theta^{k} a^{\prime}\right)$ are parallel along $T_{2}^{2}$ for some $k$, but orthogonal to the $\Omega \mathcal{R} \theta^{-k}$ and $\Omega \mathcal{R} \theta^{-k+N}$ invariant O6-planes. In this case, the orientifold projection breaks half of the supersymmetry by assigning $a_{\Omega \mathcal{R} \theta^{-k}}=1$ to one $\mathcal{N}=1$ chiral multiplet and $a_{\Omega \mathcal{R} \theta^{-k}}=-1$ to the one with opposite chirality. The

\footnotetext{
${ }^{6}$ The geometric interpretation is again strictly only valid for vanishing relative Wilson lines $\tau_{a}^{i}=\tau_{b}^{i}$ $(i=1,3)$ as in the case with three non-trivial angles.
} 


\begin{tabular}{|c|c|c|}
\hline \multicolumn{2}{|c|}{$\mathcal{N}=1$ Matter for image branes } & $\|$ on $T_{2}^{2}$ and $\perp \Omega \mathcal{R} \theta^{-k}$ \\
\hline mult. & $\left(a_{\mathbb{Z}_{2}}, a_{\Omega \mathcal{R} \theta^{-k}}, a_{\Omega \mathcal{R} \theta^{-k+N}}\right)$ & rep. \\
\hline$y_{0}^{(2)}$ & $(*, *, *)$ & $\mathbf{A n t i}_{a}^{i}+\mathbf{S y m}_{a}^{i}+$ c.c. \\
\hline$y_{\mathbb{Z}_{2}}^{(2)}$ & $(+, *, *)$ & - \\
& $(-, *, *)$ & $\mathbf{A n t i}_{a}^{i}+\mathbf{S y m}_{a}^{i}+c . c$. \\
\hline$y_{\Omega \mathcal{R} \theta^{-k}}^{(2)}$ & $(*,+, *)$ & $\overline{\mathbf{A n t i}}_{a}^{i}+\mathbf{S y m}_{a}^{i}$ \\
& $(*,-, *)$ & $\mathbf{A n t i}_{a}^{i}+\overline{\mathbf{S y m}}_{a}^{i}$ \\
\hline$y_{\Omega \mathcal{R} \theta^{-k+N}}^{(2)}$ & $(*, *,+)$ & $\mathbf{A n t i}_{a}^{i}+\overline{\mathbf{S y m}}_{a}^{i}$ \\
& $(*, *,-)$ & $\overline{\mathbf{A n t i}}_{a}^{i}+\mathbf{S y m}_{a}^{i}$ \\
\hline$y_{\mathbb{Z}_{2}+\Omega \mathcal{R} \theta^{-k}}^{(2)}$ & $(+, \pm, \pm)$ & - \\
& $(-,+,-)$ & $\overline{\mathbf{A n t i}}_{a}^{i}+\mathbf{S y m}_{a}^{i}$ \\
& $(-,-,+)$ & $\mathbf{A n t i}_{a}^{i}+\overline{\mathbf{S y m}}_{a}^{i}$ \\
\hline
\end{tabular}

Table 5: $\mathcal{N}=1$ supersymmetric sectors occurring for branes $a$ and $\left(\theta^{k} a^{\prime}\right)$ parallel along the $\mathbb{Z}_{2}$ invariant $T_{2}^{2}$ but perpendicular to the $\Omega \mathcal{R} \theta^{-k}$ and $\Omega \mathcal{R} \theta^{-k+N}$ invariant O6-planes.

resulting matter spectrum is listed in table 5. This part of the spectrum has net-chirality $\pm \frac{1}{2}\left(\left|I_{a}^{\Omega \mathcal{R} \theta^{-k},(1 \cdot 3)}\right|-\left|I_{a}^{\Omega \mathcal{R} \theta^{-k+N},(1 \cdot 3)}\right|\right)$ for antisymmetric and symmetric representations with the sign inside the parenthesis corresponding to the fact that we have identified the multiplets as being $\mathbb{Z}_{2}$ odd. This result fits nicely with (3.6) since the contribution from $T_{2}^{2}$ is

$$
N_{O 6}^{\Omega \mathcal{R} \theta^{-x},(2)}\left|I_{a}^{\Omega \mathcal{R} \theta^{-x},(2)}\right|=2
$$

for any lattice and any exponent $x .^{7}$

\subsubsection{Branes parallel along a torus where $\mathbb{Z}_{2}$ acts}

The present case applies to branes parallel on $T_{1}^{2}$ or $T_{3}^{2}$ in the $T^{6} / \mathbb{Z}_{6}^{\prime}$ background. For concreteness, we focus on the latter. As shown in table 24, the two massless multiplets have opposite chirality and opposite $\mathbb{Z}_{2}$ eigenvalue, and according to the intersection numbers in section 3.2.4, net-chiralities arise in this sector. In the absence of Wilson lines, the spectrum is given in table 6, where we assumed that both massless multiplets have the same $\Omega \mathcal{R} \theta^{-k}$ transformation. If instead the $\Omega \mathcal{R} \theta^{-k+N}$ eigenvalue is identical, the representations for $y_{\Omega \mathcal{R} \theta^{-k}}^{(3)}$ and $y_{\Omega \mathcal{R} \theta^{-k+N}}^{(3)}$ have to be exchanged while replacing $a_{\Omega \mathcal{R} \theta^{-k}} \leftrightarrow-a_{\Omega \mathcal{R} \theta^{-k+N}}$ and

\footnotetext{
${ }^{7}$ Throughout the article we use the sloppy notation $I_{a}^{\Omega \mathcal{R} \theta^{-x}}$ instead of $N_{O 6}^{\Omega \mathcal{R} \theta^{-x}} I_{a}^{\Omega \mathcal{R} \theta^{-x}}$ since for a $\mathbb{Z}_{3}$ or $\mathbb{Z}_{6}$ invariant two-torus $T_{m}^{2}$, the number of $\Omega \mathcal{R} \theta^{-x}$ invariant planes is $N_{O 6}^{\Omega \mathcal{R} \theta^{-x}},(m)=1$. However, on a $\mathbb{Z}_{2}$ invariant torus such as $T_{3}^{2}$ for $T^{6} / \mathbb{Z}_{6}^{\prime}$ we have $N_{O 6}^{\Omega \mathcal{R} \theta^{-x},(m)}=2(1-b)$, and for a $\mathbb{Z}_{4}$ invariant $T_{m}^{2}$, $N_{O 6}^{\Omega \mathcal{R} \theta^{-x},{ }^{(m)}}=1$ for $x=1,3$ on the $\mathbf{A}$ type lattice and $N_{O 6}^{\Omega \mathcal{R} \theta^{-x},(m)}=2$ for $x=0,2$. For the $\mathbf{B}$ orientation on a $\mathbb{Z}_{4}$ invariant torus, the values of $N_{O 6}^{\Omega \mathcal{R} \theta^{-x},{ }^{(m)}}$ for $x$ even and odd are exchanged compared to the $\mathbf{A}$ torus.
} 


\begin{tabular}{|c|c|c|}
\hline \multicolumn{3}{|c|}{$\mathcal{N}=1$ Matter for branes parallel on $T_{3}^{2}$} \\
\hline mult. & $\left(a_{\Omega \mathcal{R} \theta^{-k}}\right)$ & rep. \\
\hline$x_{\mathbb{Z}_{2} \text {-pairs }}^{(3)}$ & $(*)$ & $\left(\mathbf{N}_{a}^{i}, \overline{\mathbf{N}}_{b}^{j}\right)+$ c.c. \\
\hline$x_{\mathbb{Z}_{2}}^{(3)}$ & $(*)$ & $\left(\mathbf{N}_{a}^{i}, \overline{\mathbf{N}}_{b}^{i}\right)$ or $\left(\overline{\mathbf{N}}_{a}^{i}, \mathbf{N}_{b}^{j}\right)_{i \neq j}$ \\
\hline$y_{0}^{(3)}$ & $(*)$ & $\mathbf{A n t i}_{a}^{i}+\mathbf{S y m}_{a}^{i}+$ c.c. \\
\hline$y_{\mathbb{Z}_{2}}^{(3)}$ & $(*)$ & $\overline{\mathbf{A n t i}}_{a}^{i}+\overline{\mathbf{S y m}}_{a}^{i}$ \\
\hline$y_{\Omega \mathcal{R} \theta^{-k}}^{(3)}$ & $(+)$ & $\mathbf{S y m}_{a}^{i}+$ c.c. \\
& $(-)$ & $\mathbf{A n t i}_{a}^{i}+$ c.c. \\
\hline$y_{\Omega \mathcal{R} \theta^{-k+N}}^{(3)}$ & $(+)$ & $\mathbf{S y m}_{a}^{i}+\overline{\mathbf{A n t i}}_{a}^{i}$ \\
& $(-)$ & $\mathbf{A n t i}_{a}^{i}+\overline{\mathbf{S y m}}_{a}^{i}$ \\
\hline$y_{\mathbb{Z}_{2}+\Omega \mathcal{R} \theta^{-k}}^{(3)}$ & $(+)$ & $\overline{\mathbf{S y m}}_{a}^{i}$ \\
& $(-)$ & $\overline{\mathbf{A n t i}}_{a}^{i}$ \\
\hline
\end{tabular}

Table 6: $\mathcal{N}=1$ supersymmetric sectors occurring for branes $a$ and $\left(\theta^{k} b\right)$ or $\left(\theta^{k} a^{\prime}\right)$ parallel along the two-torus $T_{3}^{2}$ where $\mathbb{Z}_{2}$ acts non-trivially. The symmetric and antisymmetric representations are listed for the case of identical $\Omega \mathcal{R} \theta^{-k}$ eigenvalue for the two multiplets of opposite chirality and $\mathbb{Z}_{2}$ eigenvalue.

taking complex conjugates except for the $y_{\mathbb{Z}_{2}}^{(3)}$ entry which is independent of the orientifold projection. The generalisation to including Wilson lines along the two-torus with $\mathbb{Z}_{2}$ action and a non-vanishing angle is implemented as before by adjusting the interpretation of $x_{\mathbb{Z}_{2} \text {-pairs }}^{(3)}$ as a sum of $\mathbb{Z}_{2}$ invariant intersection points including relative signs. A relative Wilson line along the parallel direction provides a mass, see equation (3.3).

Bifundamental matter at intersections with orientifold invariant branes is computed in the same way, the $\Omega \mathcal{R} \theta^{-k}$ action on Chan-Paton labels, however, differs and is discussed in appendix $\mathrm{Q}$.

\section{Gauge couplings}

The gauge coupling constant $g_{a}$ for a gauge factor $G_{a}$ at the energy scale $\mu<M_{\text {string }}$ is at one loop given by

$$
\frac{8 \pi^{2}}{g_{a}^{2}(\mu)}=\frac{8 \pi^{2}}{g_{a, \text { string }}^{2}}+\frac{b_{a}}{2} \ln \left(\frac{M_{\text {string }}^{2}}{\mu^{2}}\right)+\frac{\Delta_{a}}{2} .
$$

The three contributions on the right hand side are

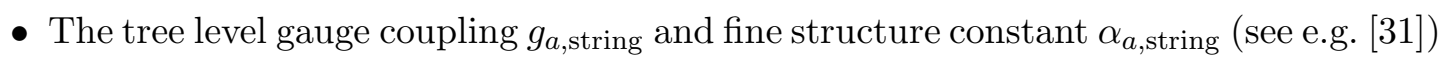

$$
\frac{1}{\alpha_{a, \text { string }}}=\frac{4 \pi}{g_{a, \text { string }}^{2}}=\frac{M_{\text {Planck }}}{2 \sqrt{2} \kappa_{a} M_{\text {string }}} \frac{V_{a}}{\sqrt{V_{6}}}
$$


where $V_{6}$ is the six dimensional compact volume, $V_{a}$ the volume of the three-cycle wrapped by brane $a$ and $\kappa_{a}=1$ for $\mathrm{SU}\left(N_{a}\right)$ gauge groups. $\kappa_{a}=2$ applies to $\operatorname{Sp}\left(2 N_{a}\right)$ and $\mathrm{SO}\left(2 N_{a}\right)$ gauge groups. The dilaton dependence has been eliminated from (4.2) by inserting the gravitational and string scales, $M_{\text {Planck }}$ and $M_{\text {string }}$, and $\alpha_{a \text {,string }}^{-1}$ depends only on the complex structure moduli. A universal one-loop correction is included in (4.2) when the redefinition of the dilaton and complex structure moduli at one-loop level is inserted 32]. At the orbifold point, all exceptional cycles have zero volume, and the volume of a fractional cycle is simply given by its toroidal part,

$$
\begin{aligned}
& V_{a}=c L_{1}^{a} \cdot L_{2}^{a} \cdot L_{3}^{a} \\
&=c\left(r_{1} r_{2} R_{1}\right)[\left(1+b^{2} \frac{R_{2}^{2}}{R_{1}^{2}}\right)\left(\tilde{a}_{1}^{2}+\tilde{a}_{1} \tilde{a}_{2}+\tilde{a}_{2}^{2}\right)+2 b \frac{R_{2}^{2}}{R_{1}^{2}}\left(\tilde{a}_{1} \tilde{a}_{3}+\frac{\tilde{a}_{1} \tilde{a}_{4}+\tilde{a}_{2} \tilde{a}_{3}}{2}+\tilde{a}_{2} \tilde{a}_{4}\right) \\
&\left.+\frac{R_{2}^{2}}{R_{1}^{2}}\left(\tilde{a}_{3}^{2}+\tilde{a}_{3} \tilde{a}_{4}+\tilde{a}_{4}^{2}\right)\right]^{1 / 2},
\end{aligned}
$$

where $r_{i}$ are the radii on $T_{i}^{2}$ for $i=1,2$ and $R_{1}, R_{2}$ those on $T_{3}^{2}$ and $\frac{\tilde{a}_{1} \tilde{a}_{4}+\tilde{a}_{2} \tilde{a}_{3}}{2}=\tilde{a}_{1} \tilde{a}_{4}=$ $\tilde{a}_{2} \tilde{a}_{3}$ with the bulk wrapping numbers $\tilde{a}_{i}$ introduced in section 2 . The constant $c$ takes care of the different normalisation of a fractional cycle compared to a toroidal one.

For the massless linear combination $\mathrm{U}(1)_{X}=\sum_{i} x_{i} \mathrm{U}(1)_{i}$ with $\mathrm{U}(1)_{i} \subset \mathrm{U}\left(N_{i}\right)$, the fine structure constant is given by ${ }^{8}$

$$
\frac{1}{\alpha_{X}}=\sum_{i} 2 N_{i} x_{i}^{2} \frac{1}{\alpha_{i}}
$$

- The running of the gauge coupling at one-loop due to massless string modes charged under $G_{a}=\mathrm{SU}\left(N_{a}\right)$ encoded in the beta function coefficient $b_{a}$ with

$$
b_{\mathrm{SU}\left(N_{a}\right)}=-N_{a}\left(3-\varphi^{\mathbf{A d j}_{a}}\right)+\sum_{b \neq a} \frac{N_{b}}{2}\left(\varphi^{a b}+\varphi^{a b^{\prime}}\right)+\frac{N_{a}-2}{2} \varphi^{\mathbf{A n t i}_{a}}+\frac{N_{a}+2}{2} \varphi^{\mathbf{S y m}_{a}} .
$$

For $\mathrm{U}(1)_{a}$ gauge groups inside $\mathrm{U}\left(N_{a}\right)$ factors, the coefficient is

$$
b_{\mathrm{U}(1)_{a}}=N_{a}\left(\sum_{b \neq a} N_{b}\left(\varphi^{a b}+\varphi^{a b^{\prime}}\right)+2\left(N_{a}+1\right) \varphi^{\mathbf{S y m}_{a}}+2\left(N_{a}-1\right) \varphi^{\mathbf{A n t i}_{a}}\right),
$$

with the beta function coefficient for a massless $\mathrm{U}(1)_{X}=\sum_{i} x_{i} \mathrm{U}(1)_{i}$ factor given by

$$
b_{X}=\sum_{i} x_{i}^{2} b_{i}+2 \sum_{i<j} N_{i} N_{j} x_{i} x_{j}\left(-\varphi^{i j}+\varphi^{i j^{\prime}}\right) .
$$

\footnotetext{
${ }^{8}$ The factor $2 N_{i}$ is due to the different prefactors of the canonical four dimensional kinetic terms for Abelian and non-Abelian gauge fields, $-\int_{\mathbb{R}^{1,3}}\left(\frac{1}{4 g_{\mathrm{U}(1)}^{2}} F_{\mathrm{U}(1)} \wedge \star F_{\mathrm{U}(1)}+\frac{1}{2 g_{\mathrm{SU}(N)}^{2}} \operatorname{tr}\left(F_{\mathrm{SU}(N)} \wedge \star F_{\mathrm{SU}(N)}\right)\right)$, with the quadratic Casimir of the fundamental representation of $\mathrm{SU}(N)$ normalised to $1 / 2$ and the fact that $\operatorname{tr}\left(F_{\mathrm{U}(N)} \wedge \star F_{\mathrm{U}(N)}\right)=\operatorname{tr}\left(F_{\mathrm{SU}(N)} \wedge \star F_{\mathrm{SU}(N)}\right)+N F_{\mathrm{U}(1)_{\mathrm{diag}}} \wedge \star F_{\mathrm{U}(1)_{\mathrm{diag}}}$ as noted e.g. in 33].
} 


\begin{tabular}{|c|c|}
\hline \multicolumn{2}{|c|}{$\mathrm{U}(3)_{a} \times \mathrm{U}(2)_{b} \times \mathrm{U}(1)_{c} \times \mathrm{U}(1)_{d}$} \\
\hline particle & $n$ \\
\hline$Q_{L}$ & $\chi^{a b}+\chi^{a b^{\prime}}$ \\
$u_{R}$ & $\chi^{a^{\prime} c}+\chi^{a^{\prime} d}$ \\
$d_{R}$ & $\chi^{a^{\prime} c^{\prime}}+\chi^{a^{\prime} d^{\prime}}+\chi^{\mathbf{A n t i}_{a}}$ \\
\hline$L$ & $\chi^{b c}+\chi^{b d}+\chi^{b^{\prime} c}+\chi^{b^{\prime} d}$ \\
$e_{R}$ & $\chi^{c d^{\prime}}+\chi^{\mathbf{S y m}_{c}}+\chi^{\mathbf{S y m}_{d}}$ \\
\hline \multicolumn{2}{|c|}{$Q_{Y}=\frac{1}{6} Q_{a}+\frac{1}{2} Q_{c}+\frac{1}{2} Q_{d}$} \\
\hline
\end{tabular}

Table 7: The fertile standard model-like configuration with $n$ generations. The number of righthanded neutrinos $\nu_{R}$ as well as the number of "chiral" Higgs candidates $\left(H_{u}, H_{d}\right)$ are left as free parameters. In the last line, the hyper charge $Q_{Y}$ is given as a linear combination of the original $\mathrm{U}(1)$ factors.

- The one-loop gauge threshold correction $\Delta_{a}$ due to charged massive string modes. This correction has been computed for bulk D6-branes in [34-36] and for rigid D6-branes in [32, 37]. For the fractional non-rigid D6-branes employed in this article, to our knowledge no explicit result has been obtained so far. Since the threshold corrections are expected to be tiny at energy scales $\mu$ well below the string scale, we are postponing their discussion to future work [30] and neglecting them at this point in our statistical analysis of vacua.

\section{Standard model constraints}

In 11], we considered the most general way to obtain $n$ standard model generations from at most four different D6-branes with initial gauge group $\mathrm{U}(3)_{a} \times \mathrm{U}(2) / \mathrm{Sp}(2)_{b} \times \mathrm{U}(1)_{c} \times \mathrm{U}(1)_{d}$ and right-handed quarks and leptons realised either as bifundamental or antisymmetric representations. We furthermore imposed supersymmetry and RR tadpole cancellation on all possible brane configurations. It turned out that only one single option of the four possibilities led to $n$ generation standard model-like spectra. The prolific configuration is displayed in table 7.9

The number of right-handed neutrinos $\nu_{R}$ and "chiral" Higgs particles ${ }^{10}$ are kept as free parameters. The standard model constraints in table 0 imply that vector like pairs

\footnotetext{
${ }^{9}$ To be exhaustive, all four possible combinations $Q_{Y}=\frac{1}{6} Q_{a} \pm \frac{1}{2} Q_{c} \pm \frac{1}{2} Q_{d}$ corresponding to permutations of orientifold image branes $c \leftrightarrow c^{\prime}$ and $d \leftrightarrow d^{\prime}$ should be considered. A check of random samples showed that all four choices have the same statistical behaviour. The standard model configurations with $Q_{Y}$ as defined in table 7 is therefore complete up to some statistical factor of the order of $\mathcal{O}(4)$.

${ }^{10}$ Only right-handed neutrinos and Higgs particles stemming from non-vanishing intersection numbers are expected to contribute to the Yukawa couplings in the superpotential. Pairs of Higgs particles $\left(H_{u}, H_{d}\right)$ are "chiral" in the sense of having different charges under massive U(1) symmetries which impose selection rules on perturbative couplings, but non-chiral w.r.t. the standard model gauge group.
} 
w.r.t. the standard model, $(\mathbf{1}, \mathbf{2})_{\mathbf{1 / 2}}+(\mathbf{1}, \mathbf{2})_{-\mathbf{1} / \mathbf{2}}$, can occur. In the absence of any other gauge group, these representations have an interpretation of Higgs pairs $H_{u}+H_{d}$, and as discussed in section 0 , in the presence of of a $B-L$ symmetry, they might either form Higgs pairs or lepton-anti-lepton pairs. In the statistical approach in section 6, possible $B-L$ symmetries are not explored, but only the number $h$ of vector like pairs w.r.t. the standard model gauge group in the $(\mathbf{1}, \mathbf{2})_{\mathbf{1} / \mathbf{2}}+(\mathbf{1}, \mathbf{2})_{-\mathbf{1} / \mathbf{2}}$ representation which stem from non-vanishing intersection numbers are computed as

$$
h=\frac{1}{2}\left(\left|\chi^{b c}\right|+\left|\chi^{b d}\right|+\left|\chi^{b^{\prime} c}\right|+\left|\chi^{b^{\prime} d}\right|-\left|\chi^{b c}+\chi^{b d}+\chi^{b^{\prime} c}+\chi^{b^{\prime} d}\right|\right) .
$$

The hyper charge $Q_{Y}$ remains massless after the generalised Green Schwarz mechanism provided that its effective three-cycle

$$
\Pi_{Y}=\frac{1}{2}\left(\Pi_{a}+\Pi_{c}+\Pi_{d}\right)
$$

is $\Omega \mathcal{R}$ invariant, i.e. $\Pi_{Y}=\Pi_{Y}^{\prime}$, which is equivalent to $\vec{Y}_{a}+\vec{Y}_{c}+\vec{Y}_{d}=0$ with the toroidal contributions $Y_{1}, Y_{2}$ defined in table 11 and corresponding entries $\left(Y_{3} \ldots Y_{6}\right)$ for the exceptional parts given in appendix $\mathrm{D}$ in equation (D.9).

While individual gauge couplings depend on the string, Planck and compactification scale as well as some numerical factor, their ratios are independent of these quantities. The quotient of strong and electro-weak fine structure constants,

$$
\frac{\alpha_{s}}{\alpha_{w}}=\frac{V_{U(2)_{b}}}{V_{U(3)_{a}}}
$$

is one characteristic quantity, the weak mixing angle $\theta_{w}$ with

$$
\sin ^{2} \theta_{w}=\frac{\alpha_{Y}}{\alpha_{Y}+\alpha_{w}}
$$

another one. We compute both parameters at tree level in section 6 .

In [31, it was further argued that if there is some underlying Pati-Salam symmetry, the fine structure constants are related by

$$
\frac{1}{\alpha_{Y}}=\frac{2}{3} \frac{1}{\alpha_{s}}+\frac{1}{\alpha_{w}}
$$

and in case of an SU(5) GUT even more constrained by

$$
\frac{1}{\alpha_{s}}=\frac{1}{\alpha_{w}}=\frac{3}{5} \frac{1}{\alpha_{Y}}
$$

which includes the previous case. The statistical evaluation in section 6.4 does not point to the presence of any of these GUT relations in the $T^{6} / \mathbb{Z}_{6}^{\prime}$ background.

In [11], also SU(5) and Pati-Salam configurations have been considered with the result that on the $T^{6} / \mathbb{Z}_{6}^{\prime}$ orbifold SU(5) models occur only with an even number of generations and some chiral states in the $\mathbf{1 5}$ representation of SU(5) whereas a Pati-Salam group arises 
only for odd numbers of generations and generically with a large number of chiral exotics. ${ }^{11}$ For the sake of completeness of GUT searches, we present in appendix $\mathrm{E}$ the solutions for trinification models which turn out to give results only for two generations and a bunch of chiral exotics.

The statistical analysis in the remainder of this article focuses on supersymmetric standard model-like spectra with the hyper charge assignment as in table 7 .

\section{Statistics}

In this section we present the results of a statistical survey of standard model-like solutions. The results have been obtained by constructing all models explicitly, such that we do not have to worry about intrinsic problems of samples or unwanted correlations [22].

\subsection{Relation between solutions on different geometries}

In [11, we found that for a given shape of the $T_{3}^{2}$ lattice (or a given $b \in\{0,1 / 2\}$ ), the number of solutions to the bulk supersymmetry and RR tadpole cancellation conditions (2.5) and (2.4) with bulk entries listed in table 1 is identical for the $\mathbf{A B}$ and $\mathbf{B B}$ geometries on $T_{1}^{2} \times T_{2}^{2}$, similarly for AA and BA. The frequency of the former is by a factor $\mathcal{O}(10)$ higher than the latter, and bulk models with $b=1 / 2$ are suppressed by a factor of 10 and 6 , respectively, compared to the $b=0$ ones. In appendix D, we prove analytically that the bulk solutions on these pairs of lattices are related by a redefinition of the complex structure modulus $\varrho \rightarrow \frac{3}{4 \varrho}$ in combination with a rotation $\pi(1 / 3,0,-1 / 2)$ of the bulk cycle wrapping numbers. This change of parameters carries over to the toroidal part of the full solutions with fractional cycles and standard model-like properties to be discussed below, where, however the suppression of AA and BA geometries is enhanced to a factor of $\mathcal{O}\left(10^{8}-10^{9}\right)$ for $b=0$ compared to $\mathbf{A B}$ and $\mathbf{B B}$, and the suppression factors for $b=1 / 2$ are $\mathcal{O}\left(10^{-4}\right)$ and $\mathcal{O}\left(10^{-6}-10^{-7}\right)$, respectively.

\subsection{Counting standard model-like solutions}

We found $4.43 \times 10^{15}$ supersymmetric standard model-like solutions with three generations and massless hyper charge $Q_{Y}$ in [11]. One or two generations and a massless $Q_{Y}$ occurs for $3.42 \times 10^{19}$ and $1.63 \times 10^{12}$ configurations, respectively, while no model is found for four or more generations, even when allowing for a massive hyper charge.

Out of the eight possible geometries only four, namely $\mathbf{A B a} / \mathbf{b}$ and $\mathbf{B B a} / \mathbf{b}$, allow for standard model-like solutions, where the variants where the third torus is tilted contribute only to models with two quark-lepton families. The situation is depicted in figure 1 .

\subsection{Complex structure dependence}

In order to compare with the analysis of [20], in figure 2 and table 8 we plot the number of standard model-like spectra in dependence of the number $n$ of generations and complex structure parameter $\varrho=\frac{\sqrt{3}}{2} \frac{R_{2}}{R_{1}}$.

\footnotetext{
${ }^{11}$ The search for Pati-Salam configurations in 11] was, however, restricted to a very specific intersection pattern and consequently there might be undetected models with less chiral exotics or $\mathrm{Sp}(2)$ gauge factors.
} 


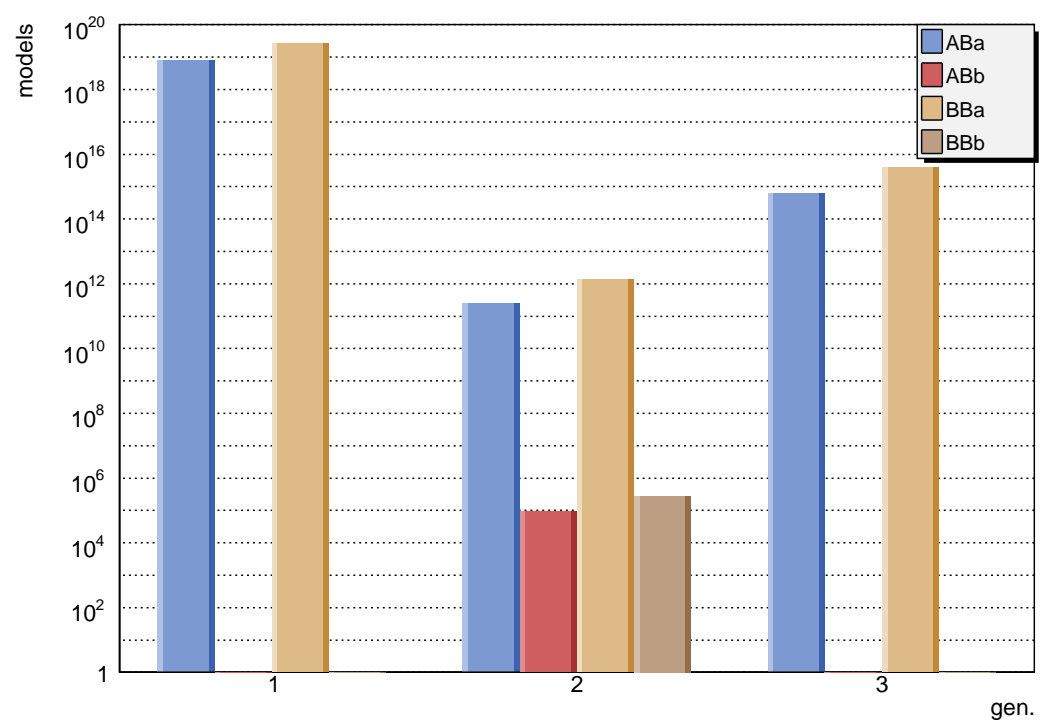

Figure 1: Distribution of supersymmetric solutions to the RR tadpole equations with gauge group and chiral matter content of the standard model for different numbers of generations and the four geometries where such models occur (the other four possibilities $\mathbf{A A a} / \mathbf{b}$ and $\mathbf{B A a} / \mathbf{b}$ do not lead to any standard model-like solutions). The hidden sectors and number of chiral exotics have not been constrained. Note that this plot includes all possibilities, more than three generations do not occur.

The results for three generation models differ from those found in [20], which were $\varrho=\frac{1}{2}, \frac{3}{2}, 1, \frac{1}{4}$ for AAa and $\frac{1}{2}, \frac{3}{2}, 3, \frac{3}{4}$ for $\mathbf{B A a}$ and 3 for $\mathbf{B B a}$ and $\frac{1}{4}$ for $\mathbf{A B a}$ in our notation, since the first two lattices in 20], AAa and BAa, are ruled out in our analysis by the RR tadpole cancellation conditions, which had not been imposed in [20]. The ABa and BBa lattices have supersymmetric solutions to the RR tadpole conditions with three chiral quark-lepton generations where our complex structure parameter $\varrho$ takes in total five different values per lattice orientation opposed to one each reported in [20] which turns out to be the one with lowest frequency in our analysis. This might be related to the fact that the constraint on three left handed quark generations $\left(\chi^{a b}, \chi^{a b^{\prime}}\right)=(2,1)$ or $(1,2)$ in [20] is more restrictive than our ansatz $\chi^{a b}+\chi^{a b^{\prime}}= \pm 3$ in table $0^{12}$

Due to the vast amount of data, in the following we will restrict our attention to one of the two geometries, ABa, where three generation models exist. For the BBa geometry, we expect essentially the same statistical behaviour as confirmed by random samples, the equivalence of bulk solutions proved in appendix $\mathrm{D}$ as well as the check that the relations and abundances of complex structure parameters for standard model-like configurations are as discussed above.

\footnotetext{
${ }^{12}$ As we will see in section 6.7 there do not exist any supersymmetric solutions to the RR tadpole conditions without chiral exotics that have $\left(\chi^{a b}, \chi^{a b^{\prime}}\right)=(2,1)$ or $(1,2)$.
} 


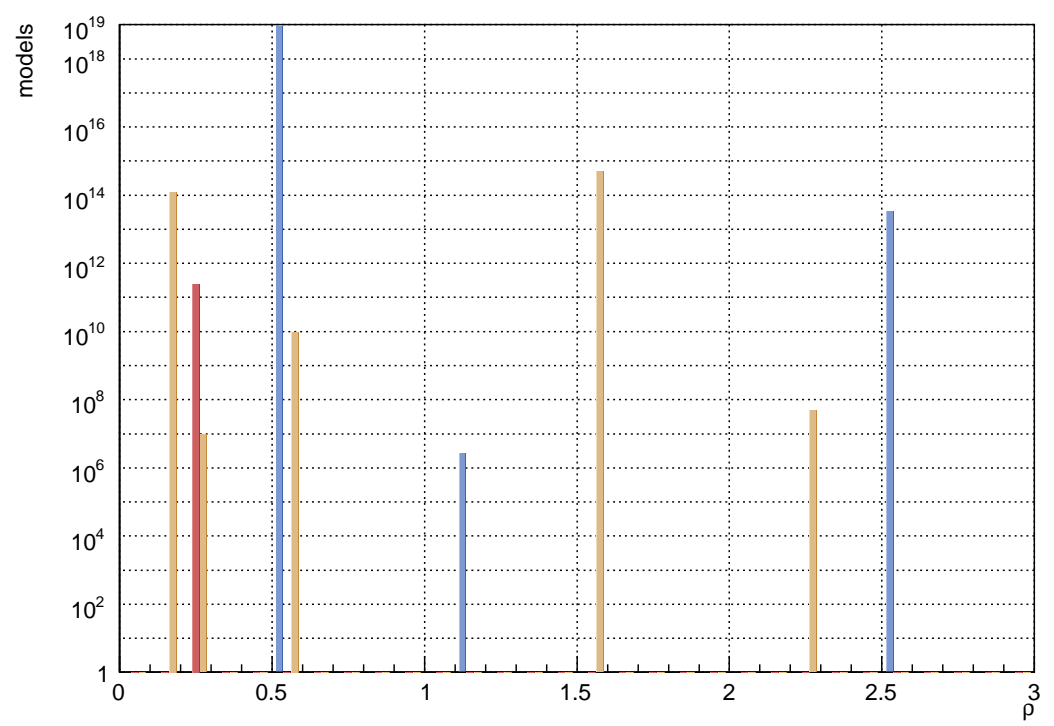

Figure 2: Distribution of the complex structure parameters $\varrho$ for the ABa geometry depending on the number of generations: yellow, red and blue correspond to three, two and one generation, respectively.

\begin{tabular}{|c|c|c|c|c|c|}
\hline \multicolumn{5}{|c|}{ Complex structure distribution } \\
\hline 1 generation & 2 generations & 3 generations \\
\hline$\varrho$ & \#models & $\varrho$ & \#models & $\varrho$ & \#models \\
\hline$\frac{1}{2}$ & $8.7 \cdot 10^{18}$ & $\frac{1}{5}$ & $2.5 \cdot 10^{11}$ & $\frac{1}{2}$ & $9.7 \cdot 10^{9}$ \\
$\frac{5}{2}$ & $3.4 \cdot 10^{13}$ & & & $\frac{1}{4}$ & $9.6 \cdot 10^{6}$ \\
$\frac{7}{6}$ & $2.7 \cdot 10^{6}$ & & $\frac{1}{6}$ & $1.2 \cdot 10^{14}$ \\
& & & $\frac{3}{2}$ & $4.9 \cdot 10^{14}$ \\
& & & $\frac{9}{4}$ & $4.9 \cdot 10^{7}$ \\
\hline
\end{tabular}

Table 8: Possible values of complex structure parameters $\varrho$ on $T_{3}^{2}$ and their abundance for $n$ generation models from the $\mathbf{A B a}$ geometry. The allowed complex structures on the $\mathbf{B B a}$ lattice are obtained by identifying $\varrho_{\mathbf{A B a}}=\frac{3}{4 \varrho_{\mathbf{B B a}}}$ with multiplicities which are by a factor $\mathcal{O}(1-10)$ bigger for each matching $\varrho$ value than the ones displayed here.

\subsection{Gauge couplings}

The ratio $\alpha_{s} / \alpha_{w}=V_{U(2)_{b}} / V_{U(3)_{a}}$ for fine structure constants takes several different values for different numbers of generations as depicted in figure 3. Also the values of the weak mixing angle (5.4) at the string scale are scattered broadly as shown in figure 4 .

This distribution of values can be compared to the measured quantities at the electro- 


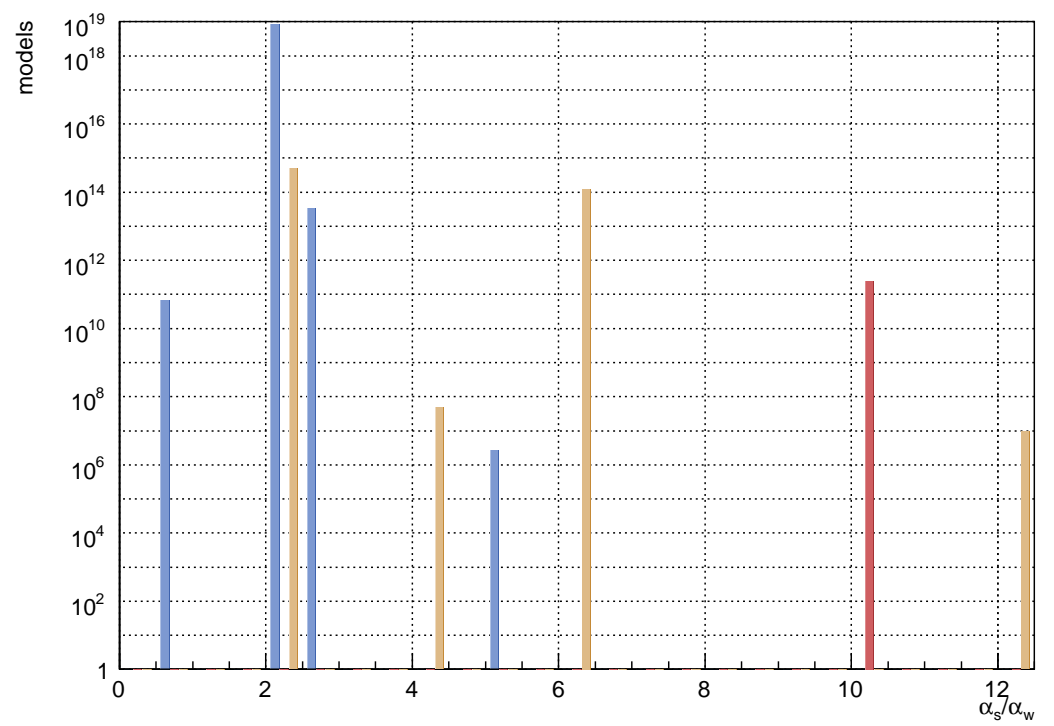

Figure 3: The tree level ratio of fine structure constants $\alpha_{s} / \alpha_{w}$ for standard-like models on the ABa lattice (yellow, red and blue correspond to three, two and one generation,respectively).

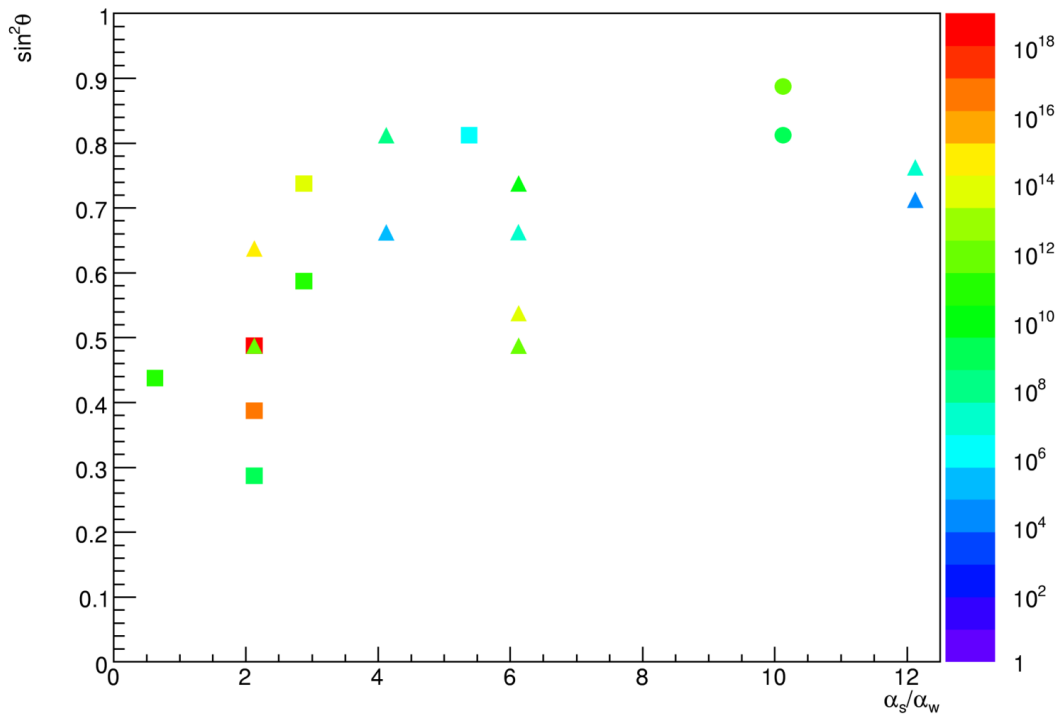

Figure 4: Combined distribution of the tree level ratios of $\alpha_{s} / \alpha_{w}$ and the weak mixing angle $\theta_{w}$ on the ABa lattice. A triangle, circle and square correspond to three, two and one generation models, respectively. Note that the colour scale on the right, which encodes the number of models with given values of $\left(\alpha_{s} / \alpha_{w}, \sin ^{2} \theta_{w}\right)$, is logarithmic.

weak scale $\left(M_{Z} \approx 91 \mathrm{GeV}\right)$,

$$
\alpha_{s}\left(M_{Z}\right) \approx 0.1, \quad \alpha_{\text {electro-magnetic }}\left(M_{Z}\right) \approx \frac{1}{128}, \quad \sin ^{2} \theta_{w}\left(M_{Z}\right) \approx 0.23,
$$


as well as results from intersecting D6-branes on other orbifolds. On $T^{6} / \mathbb{Z}_{2} \times \mathbb{Z}_{2}$ [5], the Pati-Salam relation (5.5) on gauge couplings was fulfilled in $88 \%$ of the models, but less than $3 \%$ were compatible with the SU(5) GUT relation (5.6) on the non-Abelian gauge groups. ${ }^{13}$ In the $T^{6} / \mathbb{Z}_{6}$ background with three generations [38, 10], the situation was the reverse: since all D6-branes were wrapping the same bulk cycle, $\alpha_{s}=\alpha_{w}$ was fulfilled but the hyper charge as defined in table 7 was not compatible with (5.5). On the other hand, redefining $Q_{Y}=\left(Q_{a} / 3+Q_{c}+Q_{d}+Q_{e}\right) / 2$ admitted to interpret the additional chiral particles as three generations of Higgs multiplets with non-standard Yukawa couplings and (5.5) and (5.6) were both met. The situation here differs from the other orbifolds in that $\alpha_{s} \neq \alpha_{w}$ for any number of generations, and there is no obvious relation (5.5). The values at the string scale also deviate considerably from those in equation (6.1) at the electro-weak scale, which is, however, not too surprising in view of a high string scale $M_{\text {string }} \sim 10^{16}-10^{19} \mathrm{GeV}$ and non-vanishing beta functions as well as threshold corrections for each gauge coupling.

\subsection{Number of Higgs multiplets}

The distribution of models with different numbers $h$ of "chiral" Higgs generations (plus some vector like lepton pairs in case of a massless $B-L$ symmetry), computed as given in (5.1), is shown in figure 5. In this plot we consider only models with three generations of standard model-like matter in the $\mathbf{A B a}$ geometry that have a massless hyper charge. ${ }^{14}$

It is interesting to notice that "chiral" Higgs generations (plus some vector like lepton pairs) can only appear as multiples of three. Up to the maximum value of 21 all possibilities with the exception of 15 appear. Statistically, a value of three chiral multiplets is clearly favoured, with a total number of models two orders of magnitude above all other solutions. This distribution suggests that the number of "chiral" Higgs multiplets is correlated with the constraint to obtain standard model matter with three families of quarks and leptons on this particular orbifold background and is not expected to be of any generality.

\subsection{Chiral exotics}

Within the three generation models, a wide variety of hidden sector configurations is found and almost all of the models have a large number of chiral exotics. To quantify their amount we use the total value of chiral matter multiplets between the visible and hidden sector, defined as 11

$$
\xi:=\sum_{\substack{v \in V \\ h \in H}}\left|\chi^{v h}-\chi^{v^{\prime} h}\right|,
$$

where $V=\{a, b, c, d\}$ is the set of branes in the standard model sector and $H$ contains all other stacks of (hidden) branes.

\footnotetext{
${ }^{13}$ The analysis included only one and two generation models, since no explicit three generation model was found due to the cut-off method used for the statistical treatment.

${ }^{14}$ As already mentioned earlier, models originating from the $\mathbf{B B a}$ geometry are related by a rotational symmetry, so no new results can be expected there.
} 


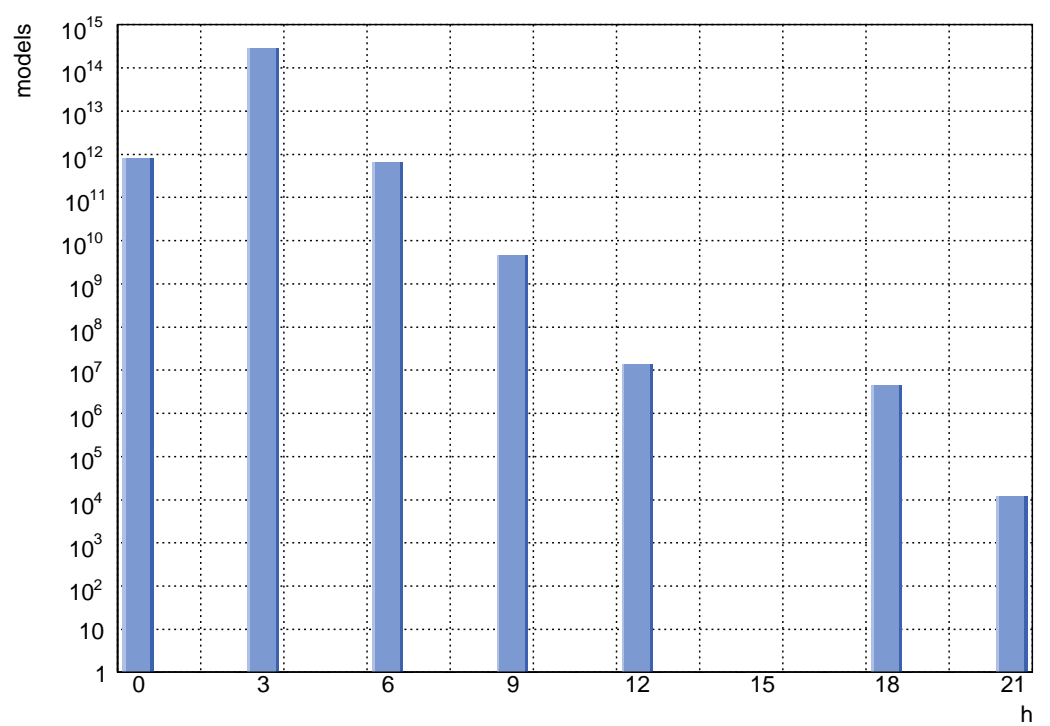

Figure 5: Number of "chiral" Higgs generation candidates (plus vector like lepton pairs in the presence of a $B-L$ symmetry) as defined in equation (5.1) for three-generation standard modellike configurations on $\mathbf{A B a}$.

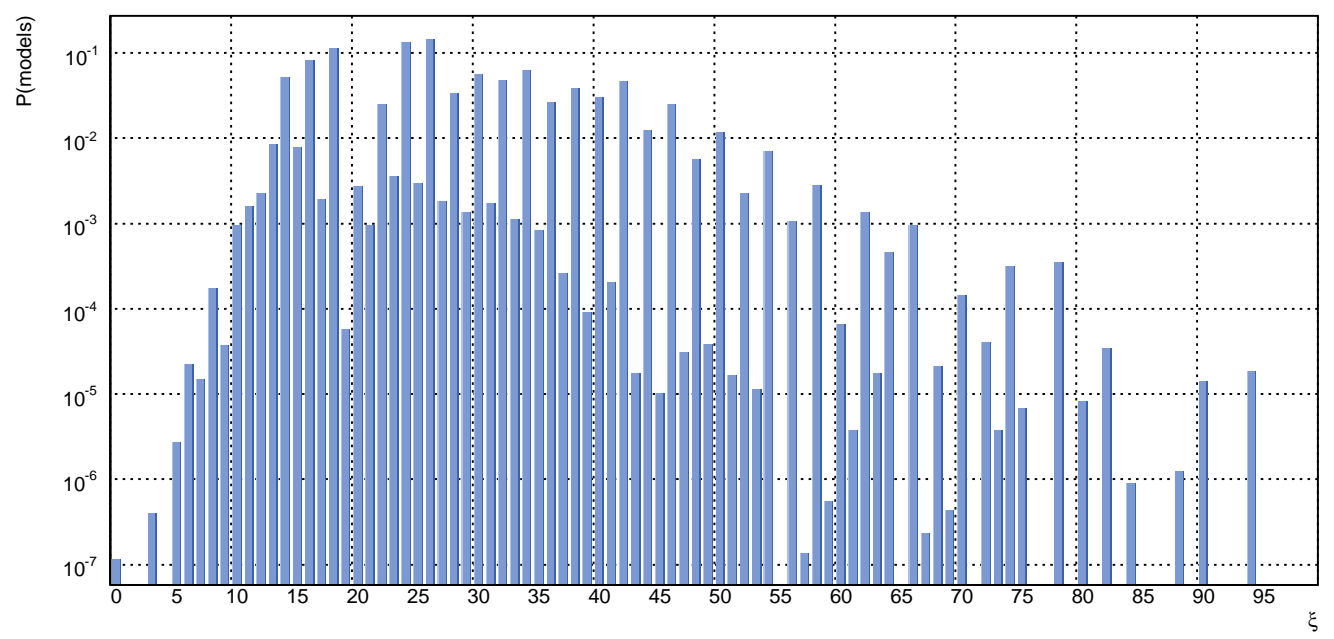

Figure 6: Normalised distribution of the total number of chiral exotics $\xi$, as defined in equation (6.2) for three-generation standard model-like configurations on ABa.

For models on the ABa torus, the statistical result is shown in figure 6. While interpreting this plot, one should keep in mind that the total amount of solutions is presented on a logarithmic scale. In particular, there is a maximum of the distribution at $\xi=26$ and an exponential falloff towards larger numbers of exotic matter. This is to be expected, since the appearance of "long" branes, admitting big intersection numbers, is exponentially 


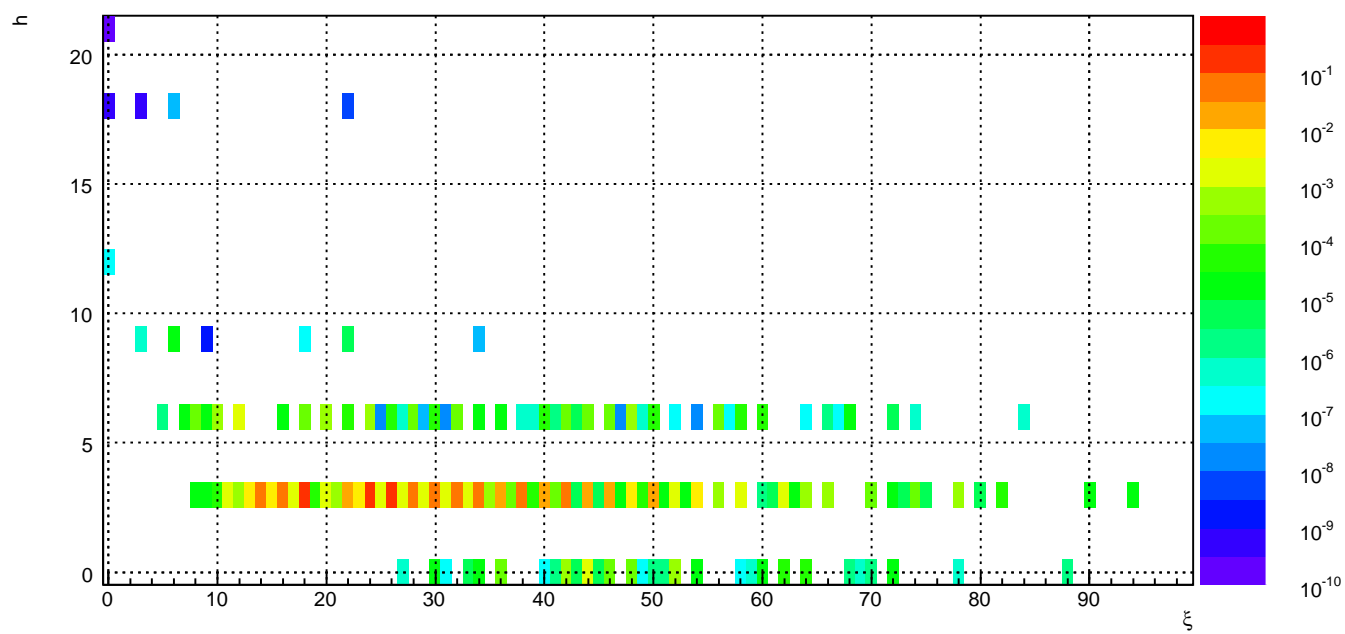

Figure 7: Relation between the number $h$ of Higgs families (plus some vector-like lepton pairs) and total number $\xi$ of chiral exotics. The normalised frequency of a given pair $(\xi, h)$ is encoded in the logarithmic colour scale on the right.

suppressed compared to shorter branes. Additionally one observes that even numbers of chiral exotics are clearly preferred. This is an artefact of the geometric set-up, much in the same way as an enhancement of even numbers for the total rank of the gauge group was observed in [5].

Although statistically largely suppressed, there do exist models without any chiral exotics. A more detailed analysis of these solutions will be the subject of section 6.7.

\subsubsection{Correlation between "chiral" Higgs multiplets and chiral exotics}

In table 9 and figure 0 the relation between the number $h$ of "chiral" Higgs generations (plus some vector-like lepton pairs) and the total number $\xi$ of chiral exotics is shown. The two quantities are obviously not independent of each other. In particular one notes that it is impossible to obtain models with an a priori small number of "chiral" Higgs multiplets and a small number of chiral exotic matter at the same time.

This result implies that even if one allows for a small number of chiral exotics, the number of Higgs multiplets will always be larger than what is desired from a phenomenological perspective unless there exist terms in the low energy effective field theory which render all but one Higgs generation sufficiently massive. A larger Higgs sector might eventually also be useful to address the mass hierarchy of standard model particles [39].

\subsection{Models without chiral exotics}

In total, 7,139,328 models without chiral exotics as defined in (6.2) exist on the ABa lattice. The vast majority of these models has the same ratios of tree level gauge couplings, namely $\alpha_{s} / \alpha_{w}=6.0$ and $\sin ^{2} \theta_{w}=0.654$, as well as twelve families of "chiral" Higgses $\left(H_{u}, H_{d}\right)$ candidates (or vector-like lepton pairs) which due to the massless $B-L$ symmetry 


\begin{tabular}{|c|c|c|c|c|c|c|c|c|c|c|c|c|c|c|}
\hline \multicolumn{15}{|c|}{ Counting of chiral exotics and chiral Higgs families } \\
\hline$\xi$ & $\mathbf{h}$ & $\mathbf{P}$ & $\xi$ & $\mathbf{h}$ & $\mathbf{P}$ & $\xi$ & $\mathbf{h}$ & $\mathbf{P}$ & $\xi$ & $\mathbf{h}$ & $\mathbf{P}$ & $\xi$ & $\mathbf{h}$ & $\mathbf{P}$ \\
\hline 0 & 12 & $1.13 \times 10^{-7}$ & 21 & 3 & $9.36 \times 10^{-4}$ & 34 & 6 & $1.53 \times 10^{-5}$ & 47 & 3 & $3.08 \times 10^{-5}$ & 62 & 0 & $5.96 \times 10^{-5}$ \\
\hline 0 & 18 & $7.87 \times 10^{-10}$ & 22 & 3 & $2.44 \times 10^{-2}$ & 34 & 9 & $3.78 \times 10^{-8}$ & 47 & 6 & $2.48 \times 10^{-8}$ & 62 & 3 & $1.31 \times 10^{-3}$ \\
\hline 0 & 21 & $1.97 \times 10^{-10}$ & 22 & 6 & $9.79 \times 10^{-5}$ & 35 & 3 & $8.38 \times 10^{-4}$ & 48 & 0 & $2.67 \times 10^{-4}$ & 63 & 3 & $1.77 \times 10^{-5}$ \\
\hline 3 & 9 & $3.97 \times 10^{-7}$ & 22 & 9 & $5.44 \times 10^{-6}$ & 36 & 0 & $2.04 \times 10^{-4}$ & 48 & 3 & $4.45 \times 10^{-3}$ & 64 & 0 & $5.71 \times 10^{-5}$ \\
\hline 3 & 18 & $6.89 \times 10^{-10}$ & 22 & 18 & $9.44 \times 10^{-9}$ & 36 & 3 & $2.59 \times 10^{-2}$ & 48 & 6 & $9.09 \times 10^{-4}$ & 64 & 3 & $3.97 \times 10^{-4}$ \\
\hline 5 & 6 & $2.72 \times 10^{-6}$ & 23 & 3 & $3.60 \times 10^{-3}$ & 36 & 6 & $2.90 \times 10^{-5}$ & 49 & 0 & $9.92 \times 10^{-8}$ & 64 & 6 & $2.86 \times 10^{-7}$ \\
\hline 6 & 9 & $2.25 \times 10^{-5}$ & 24 & 3 & $1.32 \times 10^{-1}$ & 37 & 3 & $2.62 \times 10^{-4}$ & 49 & 3 & $3.78 \times 10^{-5}$ & 66 & 3 & $9.53 \times 10^{-4}$ \\
\hline 6 & 18 & $4.56 \times 10^{-8}$ & 24 & 6 & $4.51 \times 10^{-4}$ & 38 & 3 & $3.79 \times 10^{-2}$ & 49 & 6 & $3.14 \times 10^{-7}$ & 66 & 6 & $1.26 \times 10^{-6}$ \\
\hline 7 & 6 & $1.50 \times 10^{-5}$ & 25 & 3 & $2.95 \times 10^{-3}$ & 38 & 6 & $6.19 \times 10^{-7}$ & 50 & 0 & $1.45 \times 10^{-6}$ & 67 & 6 & $2.36 \times 10^{-7}$ \\
\hline 8 & 3 & $1.60 \times 10^{-5}$ & 25 & 6 & $2.20 \times 10^{-8}$ & 39 & 3 & $9.04 \times 10^{-5}$ & 50 & 3 & $1.18 \times 10^{-2}$ & 68 & 0 & $1.14 \times 10^{-6}$ \\
\hline 8 & 6 & $1.56 \times 10^{-4}$ & 26 & 3 & $1.43 \times 10^{-1}$ & 39 & 6 & $5.78 \times 10^{-7}$ & 50 & 6 & $3.33 \times 10^{-5}$ & 68 & 6 & $2.01 \times 10^{-5}$ \\
\hline 9 & 3 & $1.09 \times 10^{-5}$ & 26 & 6 & $5.04 \times 10^{-5}$ & 40 & 0 & $1.51 \times 10^{-7}$ & 51 & 0 & $1.26 \times 10^{-6}$ & 69 & 0 & $4.23 \times 10^{-7}$ \\
\hline 9 & 6 & $2.58 \times 10^{-5}$ & 27 & 0 & $3.97 \times 10^{-7}$ & 40 & 3 & $2.99 \times 10^{-2}$ & 51 & 3 & $1.51 \times 10^{-5}$ & 70 & 0 & $1.64 \times 10^{-6}$ \\
\hline 9 & 9 & $2.75 \times 10^{-9}$ & 27 & 3 & $1.79 \times 10^{-3}$ & 40 & 6 & $5.79 \times 10^{-5}$ & 52 & 0 & $3.49 \times 10^{-4}$ & 70 & 3 & $1.41 \times 10^{-4}$ \\
\hline 10 & 3 & $8.95 \times 10^{-5}$ & 27 & 6 & $3.15 \times 10^{-7}$ & 41 & 0 & $2.31 \times 10^{-6}$ & 52 & 3 & $1.91 \times 10^{-3}$ & 72 & 0 & $1.99 \times 10^{-5}$ \\
\hline 10 & 6 & $8.56 \times 10^{-4}$ & 28 & 3 & $3.33 \times 10^{-2}$ & 41 & 3 & $1.96 \times 10^{-4}$ & 52 & 6 & $2.39 \times 10^{-7}$ & 72 & 3 & $1.56 \times 10^{-5}$ \\
\hline 11 & 3 & $1.59 \times 10^{-3}$ & 28 & 6 & $2.24 \times 10^{-4}$ & 41 & 6 & $2.18 \times 10^{-6}$ & 53 & 3 & $1.13 \times 10^{-5}$ & 72 & 6 & $4.31 \times 10^{-6}$ \\
\hline 12 & 3 & $7.91 \times 10^{-4}$ & 29 & 3 & $1.34 \times 10^{-3}$ & 42 & 0 & $9.48 \times 10^{-4}$ & 54 & 0 & $2.85 \times 10^{-5}$ & 73 & 3 & $3.77 \times 10^{-6}$ \\
\hline 12 & 6 & $1.48 \times 10^{-3}$ & 29 & 6 & $3.31 \times 10^{-8}$ & 42 & 3 & $4.48 \times 10^{-2}$ & 54 & 3 & $7.11 \times 10^{-3}$ & 74 & 3 & $3.12 \times 10^{-4}$ \\
\hline 13 & 3 & $8.46 \times 10^{-3}$ & 30 & 0 & $2.63 \times 10^{-5}$ & 42 & 6 & $1.91 \times 10^{-4}$ & 54 & 6 & $2.83 \times 10^{-8}$ & 74 & 6 & $5.19 \times 10^{-7}$ \\
\hline 14 & 3 & $5.10 \times 10^{-2}$ & 30 & 3 & $5.60 \times 10^{-2}$ & 43 & 0 & $8.73 \times 10^{-6}$ & 56 & 3 & $7.68 \times 10^{-4}$ & 75 & 3 & $6.76 \times 10^{-6}$ \\
\hline 15 & 3 & $7.70 \times 10^{-3}$ & 30 & 6 & $5.22 \times 10^{-5}$ & 43 & 3 & $4.76 \times 10^{-6}$ & 56 & 6 & $3.05 \times 10^{-4}$ & 78 & 0 & $9.09 \times 10^{-7}$ \\
\hline 16 & 3 & $8.32 \times 10^{-2}$ & 31 & 0 & $1.32 \times 10^{-7}$ & 43 & 6 & $3.67 \times 10^{-6}$ & 57 & 6 & $1.36 \times 10^{-7}$ & 78 & 3 & $3.50 \times 10^{-4}$ \\
\hline 16 & 6 & $1.63 \times 10^{-5}$ & 31 & 3 & $1.70 \times 10^{-3}$ & 44 & 0 & $1.08 \times 10^{-3}$ & 58 & 0 & $1.13 \times 10^{-7}$ & 80 & 3 & $8.31 \times 10^{-6}$ \\
\hline 17 & 3 & $1.93 \times 10^{-3}$ & 31 & 6 & $1.65 \times 10^{-8}$ & 44 & 3 & $1.11 \times 10^{-2}$ & 58 & 3 & $2.76 \times 10^{-3}$ & 82 & 3 & $3.43 \times 10^{-5}$ \\
\hline 18 & 3 & $1.13 \times 10^{-1}$ & 32 & 3 & $4.66 \times 10^{-2}$ & 44 & 6 & $2.12 \times 10^{-4}$ & 58 & 6 & $4.33 \times 10^{-5}$ & 84 & 6 & $8.92 \times 10^{-7}$ \\
\hline 18 & 6 & $1.88 \times 10^{-4}$ & 32 & 6 & $2.28 \times 10^{-4}$ & 45 & 0 & $6.16 \times 10^{-6}$ & 59 & 0 & $5.45 \times 10^{-7}$ & 88 & 0 & $1.23 \times 10^{-6}$ \\
\hline 18 & 9 & $2.26 \times 10^{-7}$ & 33 & 0 & $3.60 \times 10^{-6}$ & 45 & 3 & $3.88 \times 10^{-6}$ & 60 & 0 & $2.45 \times 10^{-5}$ & 90 & 3 & $1.43 \times 10^{-5}$ \\
\hline 19 & 3 & $5.77 \times 10^{-5}$ & 33 & 3 & $1.12 \times 10^{-3}$ & 46 & 0 & $2.11 \times 10^{-4}$ & 60 & 3 & $1.53 \times 10^{-6}$ & 94 & 3 & $1.82 \times 10^{-5}$ \\
\hline 20 & 3 & $2.10 \times 10^{-3}$ & 34 & 0 & $1.09 \times 10^{-5}$ & 46 & 3 & $2.47 \times 10^{-2}$ & 60 & 6 & $3.98 \times 10^{-5}$ & & & \\
\hline 20 & 6 & $6.04 \times 10^{-4}$ & 34 & 3 & $6.21 \times 10^{-2}$ & 46 & 6 & $2.61 \times 10^{-4}$ & 61 & 3 & $3.77 \times 10^{-6}$ & & & \\
\hline
\end{tabular}

Table 9: Normalised frequencies $P$ of three generation standard-like models for a given number $\xi$ of chiral exotics and $h$ of "chiral" Higgs families (or vector-like lepton pairs).

discussed below actually split into nine $\left(H_{u}, H_{d}\right)$ generations plus three vector-like lepton pairs. Besides that there are two smaller groups of models with different ratios of gauge couplings and more Higgs candidates as displayed in table 10.

\subsubsection{Visible sector}

The standard model sectors are all very similar in the models without chiral exotics. The chiral matter spectrum, that is responsible for the standard model particles as shown in 


\begin{tabular}{|rrr|r|}
\hline $\mathbf{h}$ & $\alpha_{\mathbf{s}} / \alpha_{\mathbf{w}}$ & $\sin ^{2} \theta_{\mathbf{w}}$ & \# models \\
\hline 12 & 6 & 0.654 & $7,077,888$ \\
18 & 4 & 0.667 & 49,152 \\
21 & 12 & 0.720 & 12,288 \\
\hline
\end{tabular}

Table 10: Number of "chiral" Higgs candidates $h$, ratio of $\alpha_{s} / \alpha_{w}$ and weak mixing angle for models without chiral exotic matter.

table 0, is identical for all models with the same number of Higgs candidates:

$$
\begin{aligned}
& h=12: \quad \chi^{a b}=0, \quad \chi^{a b^{\prime}}=3, \chi^{a c}=-3, \quad \chi^{a c^{\prime}}=-3, \quad \chi^{a d}=0, \quad \chi^{a d^{\prime}}=0, \\
& \chi^{b c}=-9, \quad \chi^{b c^{\prime}}=-9, \quad \chi^{b d}=6, \quad \chi^{b d^{\prime}}=3, \\
& \chi^{c d}=-3, \chi^{c d^{\prime}}=3 \\
& h=18: \quad \chi^{a b}=-3, \chi^{a b^{\prime}}=6, \chi^{a c}=-3, \quad \chi^{a c^{\prime}}=-3, \quad \chi^{a d}=0, \quad \chi^{a d^{\prime}}=0, \\
& \chi^{b c}=-6, \quad \chi^{b c^{\prime}}=-6, \quad \chi^{b d}=15, \quad \chi^{b d^{\prime}}=12, \\
& \chi^{c d}=-3, \chi^{c d^{\prime}}=3 \\
& h=21: \quad \chi^{a b}=0, \quad \chi^{a b^{\prime}}=3, \chi^{a c}=-3, \quad \chi^{a c^{\prime}}=-3, \quad \chi^{a d}=0, \quad \chi^{a d^{\prime}}=0, \\
& \chi^{b c}=-18, \chi^{b c^{\prime}}=-18, \chi^{b d}=6, \quad \chi^{b d^{\prime}}=3, \\
& \chi^{c d}=-3, \chi^{c d^{\prime}}=3 \text {. }
\end{aligned}
$$

Furthermore it turns out that in the absence of chiral exotics brane $c$ is always parallel to some $\Omega \mathcal{R} \theta^{-k}$ invariant plane with $\vec{Y}_{c}=0$ (as defined in table 1 and equation (D.9)) and therefore carries an $\mathrm{U}(1)_{c}$ gauge group ${ }^{15}$ which arises from the breaking of an $\mathrm{SO}(2)_{c}$ or $\mathrm{Sp}(2)_{c}$ gauge group by parallel displacement on $T_{2}^{2}$ away from the orientifold plane. Together with the condition that the hyper charge is massless (see table 7 and (5.2)) this leads to the conclusion that there exists always a massless $\mathrm{U}(1)_{B-L}$ symmetry. A more detailed discussion of this issue using explicit examples can be found in section 0 .

\subsubsection{Hidden sector}

The hidden sectors of these models are in general very small, with a maximum of three additional branes. This is a direct consequence of the orbifold geometry leading to small maximal values in the $R R$ tadpole equations, which distinguishes $T^{6} / \mathbb{Z}_{6}^{\prime}$ from other orbifolds that allow for larger hidden sectors.

The distribution of the numbers of hidden sector stacks and the ranks of their gauge groups is shown in table 11 and figure 8(a).

The models without any hidden sector correspond to those with $h=18$ or 21 (decomposing into 6 and 18 "chiral" Higgs multiplet pairs $\left(H_{u}, H_{d}\right)$ plus 12 and 3 vector-like lepton pairs, respectively, due to the $\mathrm{U}(1)_{B-L}$ symmetry) in table 10 . This implies that all models with a hidden sector have the same amount of 9 "chiral" Higgs multiplets plus 3 vector-like lepton pairs arising from non-vanishing intersection numbers as well as the same tree level values for $\alpha_{s} / \alpha_{w}$ and $\sin ^{2} \theta_{w}$ at the string scale.

\footnotetext{
${ }^{15}$ Brane $c$ in all these models belongs to type (1) in the classification of invariant branes in [11].
} 


\begin{tabular}{|l|r|r|}
\hline $\mathbf{s}$ & $\left\{\mathbf{N}_{\mathbf{i}}\right\}$ & \# models \\
\hline 0 & & 61,440 \\
\hline 1 & 1 & 147,456 \\
& 3 & 442,368 \\
\hline 2 & 2,1 & $2,433,024$ \\
\hline 3 & $1,1,1$ & $4,055,040$ \\
\hline
\end{tabular}

Table 11: Number of hidden sector branes $s$ and rank distribution $\left\{N_{i}\right\}$ of the gauge groups for standard-like models without chiral exotic matter.

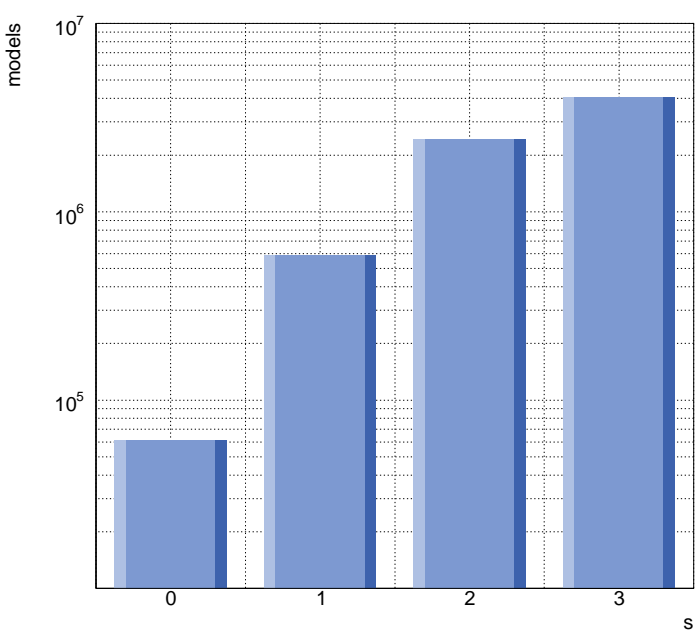

(a)

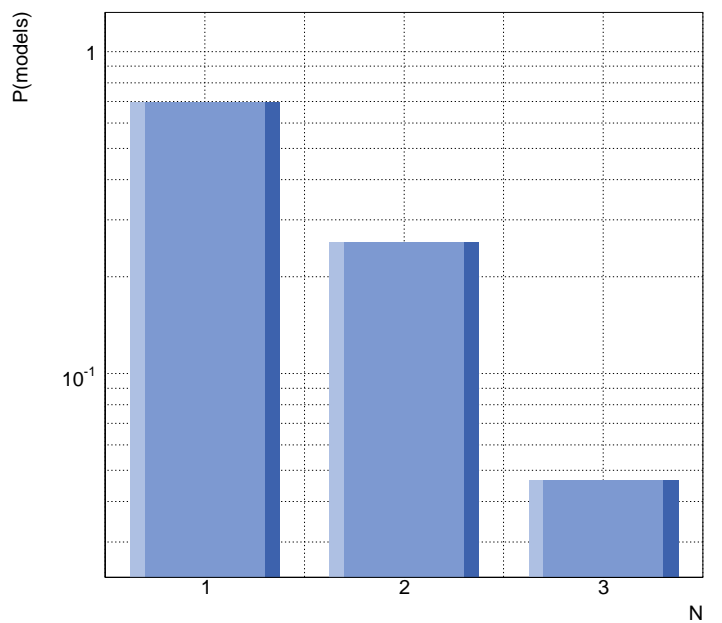

(b)

Figure 8: Distributions of properties of the hidden sector of standard model-like configurations without chiral exotic matter. (a) the number of hidden sector branes $s$; (b) probability to find a hidden sector gauge group factor of rank $N$.

\begin{tabular}{|r|r|r|}
\hline $\mathbf{N}$ & \# models & $\mathbf{P}(N)$ \\
\hline 1 & $6,635,520$ & 0.929 \\
2 & $2,433,024$ & 0.341 \\
3 & 442,368 & 0.062 \\
\hline
\end{tabular}

Table 12: Distribution of hidden sector gauge groups of rank $N$ and probability to find a gauge group of specific rank for standard-like models without chiral exotic matter.

In table 12 and figure $8(\mathrm{~b})$ the probability distribution to find a gauge group of rank $N$ within the hidden sector is shown. Besides the fact that it is cut off at a maximal rank of three, the distribution does not show differences to the more general case investigated in [11]. This is in good agreement with the results obtained for different orbifold backgrounds [5, 10], where it has also been found that the distribution of properties in the 


\begin{tabular}{|c|c|c|c|c|c|c|c|c|}
\hline & & \multicolumn{4}{|c|}{ visible sector } & \multicolumn{2}{|c|}{ hidden sector } & \multirow[b]{2}{*}{ \# models } \\
\hline $\mathbf{s}$ & $\mathbf{h}$ & $\varphi^{\mathbf{A d j}_{\mathrm{a}}}$ & $\varphi^{\mathbf{A d j}_{\mathrm{b}}}$ & $\varphi^{\mathbf{A d j}_{\mathrm{c}}}$ & $\varphi^{\mathbf{A d j}_{\mathrm{d}}}$ & $\varphi^{\mathbf{A d j}_{\mathrm{h}_{1}}}$ & $\varphi^{\mathbf{A d j}_{\mathbf{h}_{2}}} \varphi^{\mathbf{A d j}_{\mathbf{h}_{3}}}$ & \\
\hline \multirow[t]{2}{*}{0} & 18 & 2 & 4 & 4 & 10 & & & 49,152 \\
\hline & 21 & 2 & 10 & 4 & 10 & & & 12,288 \\
\hline \multirow[t]{2}{*}{1} & 12 & 2 & 10 & 4 & 10 & 2 & & 147,456 \\
\hline & 12 & 2 & 10 & 4 & 10 & 10 & & 442,368 \\
\hline 2 & 12 & 2 & 10 & 4 & 10 & 2 & 2 & $2,433,024$ \\
\hline 3 & 12 & 2 & 10 & 4 & 10 & 2 & 2 & $4,055,040$ \\
\hline
\end{tabular}

Table 13: Number of hidden sector branes $s$, number $h$ of "chiral" Higgs candidates plus vector-like lepton pairs from non-vanishing 3-cycle intersection numbers and number of adjoint representations for standard model-like spectra without chiral exotic matter. In case the hidden brane $h_{i}$ is of $S O / \operatorname{Sp}(2 N)$ type, the counting of adjoints is replaced by counting symmetric plus antisymmetric representations, $\varphi^{\mathbf{A d j}_{h_{i}}} \rightarrow \varphi^{\mathbf{S y m}_{h_{i}}}+\varphi^{\mathbf{A n t i}_{h_{i}}}$.

hidden sector does not vary significantly with respect to the distribution in the full set of solutions.

\subsubsection{Adjoint representations}

The number of adjoint representations for the different gauge groups in the visible and hidden sectors are very similar for the different realisations of standard-like models without chiral exotics. The result of a statistical analysis is shown in table 13, where for hidden $S O / \mathrm{Sp}(2 N)$ gauge groups instead of adjoints the number of symmetric plus antisymmetric representations is listed, i.e. $\varphi^{\mathbf{A d j}_{\mathrm{U}(N)}} \simeq \varphi^{\mathbf{S y m}_{S O / S p 2 N)}}+\varphi^{\mathbf{A n t i}_{S O / S p 2 N)}}$ if the toroidal wrapping numbers $\left(n_{i}, m_{i}\right)$ are identical.

This result was to be expected, since the exceptional part of the brane configuration does not play a role in determining the number of adjoints, as can be seen from the discussion in section 3.2 .

\section{Explicit standard model-like realisations}

Three examples with the chiral spectrum of the standard model, nine "chiral" Higgs generations, a massless $B-L$ symmetry and three different types of hidden sectors are discussed in section 7.1, and subsequently a model without hidden sector, 18 "chiral" Higgs generations and a massless $\mathrm{U}(1)_{B-L}$ is presented in section 7.2 .

\subsection{Three generation models with hidden sectors}

In the following, we present three explicit realisations of three generation models without chiral exotics with hidden sector gauge groups $S O / \mathrm{Sp}(6), S O / \mathrm{Sp}(4) \times S O / \mathrm{Sp}(2)$ and $S \widehat{O / \mathrm{Sp}}(2)$, respectively, ${ }^{16}$ where the bulk part of the hidden stacks $h_{3}$ and $\left(h_{2}, h_{1}\right)$ are

\footnotetext{
${ }^{16}$ The gauge group for a stack of $N$ orientifold invariant branes can be either $\mathrm{SO}(2 N)$ or $\mathrm{Sp}(2 N)$. We abbreviate this by $S O / \operatorname{Sp}(2 N)$ since the correct assignment is unknown.
} 


\begin{tabular}{|c|c|c|rrrr|}
\hline \multicolumn{7}{|c|}{ Standard model ex.: bulk brane configuration } \\
\hline brane & $\left(n_{1}, m_{1} ; n_{2}, m_{2} ; n_{3}, m_{3}\right)$ & $N$ & $\tilde{a}_{1} \equiv P, \quad \tilde{a}_{2} \equiv Q, \quad \tilde{a}_{3} \equiv U, \quad \tilde{a}_{4} \equiv V$ \\
\hline$a$ & $(1,-1 ; 1,0 ; 0,1)$ & 3 & 0, & 0, & 1, & -1 \\
\hline$b$ & $(1,1 ; 2,-1 ; 1,1)$ & 2 & 3, & 0, & 3, & 0 \\
\hline$c$ & $(1,-1 ;-1,2 ; 1,0)$ & 1 & 1, & 1, & 0, & 0 \\
\hline$d$ & $(1,1 ; 1,-2 ; 0,1)$ & 1 & 0, & 0, & 3, & -3 \\
\hline$h_{3}$ or $h_{2}+h_{1}$ & $(1,-1 ; 1,0 ; 0,1)$ & 3 or $2+1$ & 0, & 0, & 1, & -1 \\
\hline$\hat{h}_{1}$ & $(1,1 ; 1,-2 ; 0,1)$ & 1 & 0, & 0, & 3, & -3 \\
\hline
\end{tabular}

Table 14: Bulk brane configuration for standard model-like examples with hidden sectors $S O / \operatorname{Sp}(6)_{h_{3}}$ or $S O / \operatorname{Sp}(4)_{h_{2}} \times S O / S o(2)_{h_{1}}$ or $S \widehat{O O / \mathrm{Sp}}(2)_{\hat{h}_{1}}$.

\begin{tabular}{|c|ccccc|cccc|cccc|ccc|}
\hline \multicolumn{10}{|c|}{ Standard model ex.: exceptional brane configuration } \\
\hline brane & $d_{1}$, & $d_{2}$, & $d_{3}$, & $d_{4}$ & $e_{1}$, & $e_{2}$, & $e_{3}$, & $e_{4}$ & $\sigma_{1}$, & $\sigma_{2}$, & $\sigma_{5}$, & $\sigma_{6}$ & $\tau^{0}$, & $\tau^{1}$, & $\tau^{3}$ \\
\hline$a$ & 0, & 1, & -1, & 0 & 0, & 1, & -1, & 0 & $\frac{1}{2}$, & 0, & $\frac{1}{2}$, & 0 & 0, & 1, & 1 \\
\hline$b$ & -3, & 0, & -3, & 0 & 0, & 0, & 0, & 0 & $\frac{1}{2}$, & 0, & 0, & 0 & 1, & 1, & 0 \\
\hline$c$ & 0, & 0, & 3, & -3 & 0, & 0, & -3, & 3 & $\frac{1}{2}$, & 0, & 0, & $\frac{1}{2}$ & 0, & 1, & 1 \\
\hline$d$ & 0, & -1, & 1, & 0 & 0, & -1, & 1, & 0 & 0, & 0, & $\frac{1}{2}$, & 0 & 0, & 1, & 1 \\
\hline$h_{3}$ or $h_{2}$ & 1, & 0, & 0, & 1 & -1, & 0, & 0, & -1 & 0, & 0, & 0, & 0 & 0, & 1, & 0 \\
$h_{1}$ & & & & & & & & & & & 1, & 0, & 0 \\
\hline$\hat{h}_{1}$ & 3, & 0, & 0, & 3 & -3, & 0, & 0, & -3 & $\frac{1}{2}$, & 0, & 0, & 0 & 0, & 1, & 0 \\
\hline
\end{tabular}

Table 15: Exceptional brane configuration for standard model-like examples with different hidden sectors.

identical and the complex structure is $\varrho=1 / 2$ for all three choices of hidden sectors. The bulk configuration is given in table 14 and the exceptional part with displacements $\sigma_{i}$ and Wilson lines $\tau^{j}$ in table 15 . The hidden sector brane $h_{1}$ differs from $h_{3}$ and $h_{2}$ only in the assignment of $\left(\tau^{0}, \tau^{1}\right)$.

Although brane $c$ is parallel to the $\Omega \mathcal{R} \theta^{-4}$ plane with also the exceptional contributions orientifold invariant and in the classification of [11] corresponds to brane (1c) with $\mathrm{SO}(2)_{c}$ or $\operatorname{Sp}(2)_{c}$ gauge group, we assume here that brane $c$ is displaced on the $\mathbb{Z}_{2}$ invariant torus $T_{2}^{2}$ and the gauge group broken to $\mathrm{U}(1)_{c}$. We list here all states which arise in the decomposition $S O / \mathrm{Sp}(2)_{c} \rightarrow \mathrm{U}(1)_{c}$, namely $\mathbf{A n t i}_{c} \rightarrow \mathbf{1}_{0}$ and $\mathbf{S y m}_{c} \rightarrow \mathbf{1}_{0}+\mathbf{1}_{2}+\mathbf{1}_{-2}$, however, one should keep in mind that by parallely separating brane $c$ and $\left(\theta c^{\prime}\right)$ on $T_{2}^{2}$, at least one of the final states acquires a mass proportional to the distance of $c$ and $\left(\theta c^{\prime}\right)$. The intersection numbers are then given in table 16, and also the chiral plus non-chiral part of the spectrum only charged under the observable sector involving branes $a, b, c$ and $d$ in table 17 is universal.

The models differ in the purely non-chiral part of the spectrum with hidden sector charges. Their multiplicities are given in table 18. Branes $h_{i}$ are orthogonal to the 


\begin{tabular}{|c|r|r|r|r|r|r|r|r|r|r|}
\hline \multicolumn{10}{|c|}{ Standard model ex.: chiral matter spectrum } \\
\hline brane & $\chi^{\text {Anti }}$ & $\chi^{\text {Sym }}$ & $\chi^{\cdot \mathbf{b}}$ & $\chi^{\cdot \mathbf{c}}$ & $\chi^{\cdot \mathbf{d}}$ & $\chi^{\cdot \mathbf{b}^{\prime}}$ & $\chi^{\cdot \mathbf{c}^{\prime}}$ & $\chi^{\cdot \mathbf{d}^{\prime}}$ & $\chi^{\cdot \mathbf{h}}$ & $\chi^{\cdot \mathbf{h}^{\prime}}$ \\
\hline$a$ & 0 & 0 & 0 & $-3\left(d_{R}\right)$ & 0 & $3\left(Q_{L}\right)$ & $-3\left(u_{R}\right)$ & 0 & 0 & 0 \\
\hline$b$ & 0 & 0 & & $-9\left(H_{u}\right)$ & $6(L)$ & & $-9\left(H_{d}\right)$ & $3(\bar{L})$ & 0 & 0 \\
\hline$c$ & 0 & 0 & \multicolumn{10}{|c|}{} & $-3\left(\nu_{R}\right)$ & & & $3\left(e_{R}\right)$ & 0 & 0 \\
\hline$d$ & 0 & 0 & \multicolumn{10}{|c|}{} & & 0 & 0 \\
\hline
\end{tabular}

Table 16: The chiral spectrum $[C]$ derived from non-vanishing 3-cycle intersection numbers is identical for all three configurations with hidden branes $h_{3}$ or $h_{2}+h_{1}$ or $\hat{h}_{1}$. The identifications with standard model-like particles are given in parenthesis, see also equation (7.2).

\begin{tabular}{|c|c|c|c|c|c|c|c|c|c|}
\hline \multicolumn{10}{|c|}{ Standard model ex.: complete observable matter spectrum } \\
\hline brane & $\varphi^{\text {Adj }}$ & $\varphi^{\text {Anti }}$ & $\varphi^{\text {Sym }}$ & $\varphi^{\cdot \mathbf{b}}$ & $\varphi^{\cdot \mathbf{c}}$ & $\varphi^{\cdot \mathbf{d}}$ & $\varphi^{\cdot \mathbf{b}^{\prime}}$ & $\varphi^{\cdot \mathbf{c}^{\prime}}$ & $\varphi^{\cdot \mathbf{d}^{\prime}}$ \\
\hline$a$ & 2 & 6 & 0 & 0 & 3 & 6 & 5 & 3 & 6 \\
\hline$b$ & 10 & 8 & 12 & & 11 & 8 & & 11 & 7 \\
\hline$c$ & 4 & $(2 x)$ & $8-2 x$ & & 5 & & 5 \\
\hline$d$ & 10 & $(22)$ & 0 & \multicolumn{10}{|c|}{} \\
\hline
\end{tabular}

Table 17: The universal chiral plus non-chiral matter spectrum of the standard model-like examples with only charges under branes $a, b, c, d$. The models differ in their hidden sector gauge groups and non-chiral matter charged under them as listed in table 18. $0 \leq x \leq 4$ depends on the unknown $\Omega \mathcal{R} \theta^{-k}$ eigenvalues of the massless states at $c\left(\theta^{k-1} c\right)$ intersections.

$\Omega \mathcal{R} \theta^{-1+3 k}$ invariant planes on $T_{2}^{2}$ and carry $S O / \mathrm{Sp}(2 i)$ gauge factors ${ }^{17}$ whereas $\hat{h}_{1}$ is parallel to the $\Omega \mathcal{R} \theta^{-1}$ plane on all three tori corresponding to brane (2a) in the classification of 11] providing a hidden $\widehat{\mathrm{SO}(2)}_{\hat{h}_{1}}$ or $\widehat{\mathrm{Sp}(2)}_{\hat{h}_{1}}$ gauge factor. In the observable sector, out of the four $\mathrm{U}(1)$ factors, two stay massless. We choose the hyper charge $Y$ and $B-L$ symmetry as basis,

$$
Q_{Y}=\frac{1}{6} Q_{a}+\frac{1}{2} Q_{c}+\frac{1}{2} Q_{d}, \quad Q_{B-L}=\frac{1}{3} Q_{a}+Q_{d}
$$

The hidden sector gauge groups are either of type $\mathrm{SO}(2 N)$ or $\mathrm{Sp}(2 N)$. When broken to $\mathrm{U}(N)$, the Abelian part will stay massless. Remember that the transverse degrees of

\footnotetext{
${ }^{17}$ The classification (1) and (2) of D6-branes in table 20 of 11 with $\mathrm{SO}(2 N)$ or $\mathrm{Sp}(2 N)$ gauge groups comprised only those parallel to some $\Omega \mathcal{R} \theta^{-k}$ planes along all two-tori. Branes $h_{i}$ are instead orthogonal to some $\Omega \mathcal{R} \theta^{-k}$ invariant plane on $T_{2}^{2}$ and also orthogonal on either $T_{1}^{2}$ or $T_{3}^{2}$ while being parallel to the same orientifold plane on the remaining two-torus, a case overlooked before. One might worry about a new Ktheory constraint from the two new kinds of invariant branes (3) and (4), but it turns out that it is trivially fulfilled also in these cases since the line of argument in [11] involved combinatorics of odd and even numbers only, and, e.g., $\Pi_{\hat{h}_{1}}=3 \Pi_{h_{i}}$ as well as $\left(n_{j}^{\hat{h}_{1}}, m_{j}^{\hat{h}_{1}}\right)=\left(n_{j}^{h_{i}}, m_{j}^{h_{i}}\right) \bmod 2$ do not alter this reasoning. We had furthermore verified explicitly in [11] that the invariant branes (1) and (2) do not lead to any standard model-like solutions with an $\operatorname{Sp}(2)_{b}$ gauge factor. There might potentially be such configurations with the new invariant branes of type (3) or (4), but since it is at present not clear if symplectic or orthogonal gauge factors arise, this option is not taken into account in the present analysis.
} 


\begin{tabular}{|c|c|c|c|c|c|c|}
\hline \multicolumn{6}{|c|}{ Standard model ex.: non-chiral exotic matter } \\
\hline brane & $\varphi^{\cdot \mathbf{h}_{\mathbf{3}}}=\varphi^{\cdot \mathbf{h}_{\mathbf{2}}}$ & $\varphi^{\cdot \mathbf{h}_{\mathbf{1}}}$ & $\varphi^{\cdot \hat{\mathbf{h}}_{1}}$ & brane & $\varphi^{\text {Anti }}$ & $\varphi^{\text {Sym }}$ \\
\hline$a$ & 0 & 0 & 0 & $h_{3}, h_{2}, h_{1}$ & $z$ & $2-z$ \\
\hline$b$ & 2 & 2 & 8 & $\hat{h}_{1}$ & $y$ & $10-y$ \\
\hline$c$ & 2 & 2 & 10 & & & \\
\hline$d$ & 0 & 0 & 0 & \multicolumn{5}{|l}{} \\
\hline$h_{2}$ & & 2 & & \multicolumn{5}{l}{} \\
\hline
\end{tabular}

Table 18: Matter at intersection with hidden branes for the three different models with hidden sector gauge groups $S O / \mathrm{Sp}(6), S O / \mathrm{Sp}(4) \times S O / \mathrm{Sp}(2)$ and $S O / \mathrm{Sp}(2)$ corresponding to branes $h_{3}$, $h_{2}+h_{1}$ and $\hat{h}_{1}$, respectively. $0 \leq z \leq 2$ and $0 \leq y \leq 10$ depend on the undetermined $\Omega \mathcal{R} \theta^{-k}$ eigenvalues of massless states at $h_{i}\left(\theta^{k-1} h_{i}\right)$ and $\hat{h}_{1}\left(\theta^{k-1} \hat{h}_{1}\right)$ intersections, respectively.

freedom of the two massive $\mathrm{U}(1)$ factors, i.e. $Q_{b}$ and $3 Q_{a}-Q_{d}$, are provided by uncharged closed string RR states and that the supersymmetry conditions (2.5) imply that no FayetIliopoulos term appears.

The (open string) matter spectrum thus consists of three sectors, $[C]+\left[V_{U}\right]+\left[V_{H}\right]$, where $[C]$ contains all (chiral) matter derived from non-vanishing 3-cycle intersection numbers, $\left[V_{U}\right]$ the vector like matter occurring in all three models (these are the adjoint representations and $\mathcal{N}=2$ supersymmetric sectors in complex representations $\mathbf{R}+$ c.c.) and $\left[V_{H}\right]$ the vector like matter which differs for the three choices of hidden sectors (it contains symmetric and antisymmetric representations of the hidden $S O / \operatorname{Sp}(2 N)$ gauge factors as well as $\mathcal{N}=2$ sectors in complex representations $\mathbf{R}+$ c.c.):

1. The chiral spectrum of $\left((S) \mathrm{U}(3)_{a} \times(S) U(2)_{b}\right)_{Q_{Y} \times Q_{B-L}}$ which is identical in all models. In order to make the perturbatively allowed pattern of Yukawa couplings more obvious, we also list the charges under the unphysical $\mathrm{U}(1) s$ as upper indices ${ }^{\left(Q_{c}, Q_{d}\right)}$. All states in this sector form $\mathcal{N}=1$ multiplets at intersections of two different stacks of branes. For shortness, the (trivial) representation under the hidden gauge group is not listed,

$$
\begin{aligned}
{[C]=3 \times[} & (\mathbf{3}, \mathbf{2})_{\mathbf{1} / \mathbf{6}, \mathbf{1} / \mathbf{3}}^{(0,0)}+(\overline{\mathbf{3}}, \mathbf{1})_{\mathbf{1} / \mathbf{3},-\mathbf{1} / \mathbf{3}}^{(1,0)}+(\overline{\mathbf{3}}, \mathbf{1})_{-\mathbf{2} / \mathbf{3},-\mathbf{1} / \mathbf{3}}^{(-1,0)} \\
& +(\mathbf{1}, \mathbf{1})_{\mathbf{1}, \mathbf{1}}^{(1,1)}+(\mathbf{1}, \mathbf{1})_{\mathbf{0}, \mathbf{1}}^{(-1,1)}+2 \times(\mathbf{1}, \mathbf{2})_{-\mathbf{1} / \mathbf{2},-\mathbf{1}}^{(0,-1)} \\
& \left.+(\mathbf{1}, \mathbf{2})_{\mathbf{1} / \mathbf{2}, \mathbf{1}}^{(0,1)}+3 \times(\mathbf{1}, \overline{\mathbf{2}})_{-\mathbf{1} / \mathbf{2}, \mathbf{0}}^{(-1,0)}+3 \times(\mathbf{1}, \overline{\mathbf{2}})_{\mathbf{1} / \mathbf{2}, \mathbf{0}}^{(1,0)}\right] \\
\equiv 3 \times & {\left[Q_{L}+d_{R}+u_{R}+e_{R}+\nu_{R}+2 \times L+\bar{L}\right]+9 \times\left[H_{d}+H_{u}\right], }
\end{aligned}
$$

with an net-number of three chiral lepton and nine $\left(H_{u}, H_{d}\right)$ families. The $B-L$ charge reveals that there are furthermore three lepton-anti-lepton generations.

One can read off that the standard combinations for Yukawa couplings involving

$$
u_{R} \cdot Q_{L} \cdot H_{u}, \quad d_{R} \cdot Q_{L} \cdot H_{d}, \quad e_{R} \cdot L \cdot H_{d} \quad \text { and } \quad \nu_{R} \cdot L \cdot H_{u}
$$


are perturbatively allowed, whereas a $\mu$ term type combination $H_{u} \cdot H_{d}$ is perturbatively forbidden by the $\mathrm{U}(1)$ symmetry inside $\mathrm{U}(2)_{b}$. Since global (anomalous) symmetries are generally broken by non-perturbative effects, we expect an exponentially suppressed $\mu$-term to be generated by instanton corrections. Contrarily, a right-handed neutrino Majorana mass term and quartic coupling appearing in the see-saw mechanism,

$$
\nu_{R} \cdot \nu_{R} \text { and } L \cdot L \cdot H_{u} \cdot H_{u},
$$

are forbidden by the massless $B-L$ symmetry.

2. The universal part of the non-chiral spectrum containing matter only charged under stacks $a, b, c, d$ consists of adjoints in the first line and $\mathcal{N}=2$ supersymmetric sectors in the second to fifth line consisting of bifundamental, symmetric and antisymmetric representations, the latter two being denoted by lower indices $S$ and $A$, respectively. We list the $\mathcal{N}=2$ part of the spectrum in complex representations in square brackets in the form of chiral representations +c.c. (complex conjugate) where the "+c.c." applies to all representations inside the square bracket.

$$
\begin{aligned}
{\left[V_{U}\right]=2 \times } & (\mathbf{8}, \mathbf{1})_{\mathbf{0}, \mathbf{0}}^{(0,0)}+10 \times(\mathbf{1}, \mathbf{3})_{\mathbf{0}, \mathbf{0}}^{(0,0)}+26 \times(\mathbf{1}, \mathbf{1})_{\mathbf{0}, \mathbf{0}}^{(0,0)} \\
+ & {\left[(\mathbf{3}, \mathbf{2})_{\mathbf{1} / \mathbf{6}, \mathbf{1} / \mathbf{3}}^{(0,0)}+3 \times(\overline{\mathbf{3}}, \mathbf{1})_{\mathbf{1 / 3}, \mathbf{2} / \mathbf{3}}^{(0,1)}+3 \times(\overline{\mathbf{3}}, \mathbf{1})_{-\mathbf{2} / \mathbf{3},-\mathbf{4} / \mathbf{3}}^{(0,-1)}\right.} \\
& +3 \times\left(\overline{\mathbf{3}}_{A}, \mathbf{1}\right)_{\mathbf{1} / \mathbf{3}, \mathbf{2} / \mathbf{3}}^{(0,0)}+4 \times\left(\mathbf{1}, \mathbf{1}_{A}\right)_{\mathbf{0}, \mathbf{0}}^{(0,0)}+6 \times\left(\mathbf{1}, \mathbf{3}_{S}\right)_{\mathbf{0}, \mathbf{0}}^{(0,0)} \\
& +(\mathbf{1}, \overline{\mathbf{2}})_{-\mathbf{1 / 2 , \mathbf { 0 }}}^{(-1,0)}+(\mathbf{1}, \mathbf{2})_{-\mathbf{1} / \mathbf{2},-\mathbf{1}}^{(0,-1)}+(\mathbf{1}, \overline{\mathbf{2}})_{\mathbf{1} / \mathbf{2}, \mathbf{0}}^{(1,0)}+2 \times(\mathbf{1}, \mathbf{2})_{\mathbf{1} / \mathbf{2}, \mathbf{1}}^{(0,1)} \\
& \left.+(\mathbf{1}, \mathbf{1})_{\mathbf{1}, \mathbf{1}}^{(1,1)}+(\mathbf{1}, \mathbf{1})_{\mathbf{0}, \mathbf{1}}^{(-1,1)}+(4-x) \times(\mathbf{1}, \mathbf{1})_{\mathbf{1 , 0}}^{(2,0)}+\text { c.c. }\right]
\end{aligned}
$$

The parameter $0 \leq x \leq 4$ depends on the undetermined $\Omega \mathcal{R} \theta^{-k}$ eigenvalues of the massless states on brane $c$.

The vector-like pairs $\left[(\mathbf{1}, \mathbf{1})_{\mathbf{0}, \mathbf{1}}^{(-1,1)}+\right.$ c.c. $]$ might potentially trigger the breaking of $\mathrm{U}(1)_{B-L}$ without a simultaneous supersymmetry breaking.

3. The non-universal part containing matter charged under the hidden sector gauge group where hidden sector representations are the last one or two entries separated from the observable sector charges by a semicolon. Remarkably, no matter arises at intersections of brane $a$ and $d$ with the hidden branes leading to $Q_{B-L}=0$ in this sector. The notation for the non-chiral matter is analogous to the one for the universal part of the spectrum with antisymmetric and symmetric representations of $S O / \mathrm{Sp}(2 N)$ replacing the adjoints of $\mathrm{U}(N)$.

(a) For the hidden gauge group $S O / \mathrm{Sp}(6)_{h_{3}}$

$$
\begin{aligned}
{\left[V_{H_{1}}\right]=} & z \times(\mathbf{1}, \mathbf{1} ; \mathbf{1 5})_{\mathbf{0}, \mathbf{0}}^{(0,0)}+(2-z) \times(\mathbf{1}, \mathbf{1} ; \mathbf{2 1})_{\mathbf{0}, \mathbf{0}}^{(0,0)} \\
& +\left[(\mathbf{1}, \mathbf{2} ; \mathbf{6})_{\mathbf{0}, \mathbf{0}}^{(0,0)}+(\mathbf{1}, \mathbf{1} ; \mathbf{6})_{\mathbf{1} / \mathbf{2}, \mathbf{0}}^{(1,0)}+\text { c.c. }\right]
\end{aligned}
$$


(b) Or for hidden sector gauge factors $S O / \mathrm{Sp}(4)_{h_{2}} \times S O / \mathrm{Sp}(2)_{h_{1}}$

$$
\begin{aligned}
{\left[V_{H_{2}}\right]=} & z \times(\mathbf{1}, \mathbf{1} ; \mathbf{6}, \mathbf{1})_{\mathbf{0}, \mathbf{0}}^{(0,0)}+(2-z) \times(\mathbf{1}, \mathbf{1} ; \mathbf{1 0}, \mathbf{1})_{\mathbf{0}, \mathbf{0}}^{(0,0)} \\
& +z \times(\mathbf{1}, \mathbf{1} ; \mathbf{1}, \mathbf{1})_{\mathbf{0}, \mathbf{0}}^{(0,0)}+(2-z) \times(\mathbf{1}, \mathbf{1} ; \mathbf{1}, \mathbf{3})_{\mathbf{0}, \mathbf{0}}^{(0,0)} \\
& +2 \times(\mathbf{1}, \mathbf{1} ; \mathbf{4}, \mathbf{2})_{\mathbf{0}, \mathbf{0}}^{(0,0)} \\
+ & {\left[(\mathbf{1}, \mathbf{2} ; \mathbf{4}, \mathbf{1})_{\mathbf{0}, \mathbf{0}}^{(0,0)}+(\mathbf{1}, \mathbf{2} ; \mathbf{1}, \mathbf{2})_{\mathbf{0}, \mathbf{0}}^{(0,0)}\right.} \\
& \left.+(\mathbf{1}, \mathbf{1} ; \mathbf{4}, \mathbf{1})_{\mathbf{1} / \mathbf{2}, \mathbf{0} ; \mathbf{0}}^{(1,0)}+(\mathbf{1}, \mathbf{1} ; \mathbf{1}, \mathbf{2})_{\mathbf{1} / \mathbf{2}, \mathbf{0}}^{(1,0)}+\text { c.c. }\right] .
\end{aligned}
$$

(c) Or for a hidden $S \widehat{O / \mathrm{Sp}}(2)_{\hat{h}_{1}}$

$$
\begin{aligned}
{\left[V_{H_{3}}\right]=} & y \times\left(\mathbf{1}, \mathbf{1} ; \mathbf{1}_{A}\right)_{\mathbf{0}, \mathbf{0}}^{(0,0)}+(10-y) \times\left(\mathbf{1}, \mathbf{1} ; \mathbf{3}_{S}\right)_{\mathbf{0}, \mathbf{0}}^{(0,0)} \\
& +\left[4 \times(\mathbf{1}, \mathbf{2} ; \mathbf{2})_{\mathbf{0}, \mathbf{0}}^{(0,0)}+5 \times(\mathbf{1}, \mathbf{1} ; \mathbf{2})_{\mathbf{1} / \mathbf{2}, \mathbf{0}}^{(1,0)}+\text { c.c. }\right],
\end{aligned}
$$

where the parameters $z$ and $y$ depend on the undetermined $\Omega \mathcal{R} \theta^{-k}$ eigenvalues of the massless states at intersections of $h_{i}$ and $\hat{h}_{1}$ with their orbifold images $\left(\theta^{k-1} h_{i}\right)$ and $\left(\theta^{k-1} \hat{h}_{1}\right)$, respectively.

These models belong to the class of spectra with $9+3=12$ "chiral" Higgs plus leptonanti-lepton families and no chiral exotics whose tree level ratio of gauge couplings are given in table 10. The beta function coefficients for these models can be computed explicitly,

$$
\begin{array}{rlrl}
b_{\mathrm{SU}(3)_{a}}=14, & b_{\mathrm{SU}(2)_{b}}= & \left\{\begin{array}{ll}
70 & h_{3}, h_{2}+h_{1} \\
72 & \hat{h}_{1}
\end{array},\right. \\
b_{\mathrm{U}(1)_{B-L}}=\frac{280}{3}, & b_{\mathrm{U}(1)_{Y}}=\left\{\begin{array}{ll}
\frac{305}{6}-2 x h_{3}, h_{2}+h_{1} \\
\frac{157}{3}-2 x & \hat{h}_{1}
\end{array} .\right.
\end{array}
$$

Although interactions have to our knowledge not yet been computed for the type of fractional D6-branes used in the construction, some of the non-chiral matter in the $\left[V_{U}\right]+\left[V_{H}\right]$ sectors can be made massive by simply parallely displacing the one-cycles along $T_{2}^{2}$ away from the origin and each other. The parallel transport preserves the supersymmetry conditions (2.5), and the situation is depicted in figure 9 for branes $b$ and $c$.

The mass scale is again set by the continuously variable distance $\Delta \sigma^{2}$ according to equation (3.3).

After displacing all brane stacks relative to each other along $T_{2}^{2}$, the chiral sector $[C]$ remains unchanged, but the vector like matter sector $\left[V_{U}\right]$ is reduced (with $0 \leq \tilde{x} \leq 3$ ),

$$
\begin{aligned}
{\left[V_{U}\right]^{\left(\Delta \sigma^{2}\right)}=2 \times } & (\mathbf{8}, \mathbf{1})_{\mathbf{0}, \mathbf{0}}^{(0,0)}+10 \times(\mathbf{1}, \mathbf{3})_{\mathbf{0}, \mathbf{0}}^{(0,0)}+25 \times(\mathbf{1}, \mathbf{1})_{\mathbf{0}, \mathbf{0}}^{(0,0)} \\
+ & {\left[(\mathbf{3}, \mathbf{2})_{\mathbf{1} / \mathbf{6}, \mathbf{1} / \mathbf{3}}^{(0,0)}+3 \times(\overline{\mathbf{3}}, \mathbf{1})_{\mathbf{1} / \mathbf{3}, \mathbf{2} / \mathbf{3}}^{(0,1)}+3 \times(\overline{\mathbf{3}}, \mathbf{1})_{-\mathbf{2} / \mathbf{3},-\mathbf{4} / \mathbf{3}}^{(0,-1)}\right.} \\
& \left.+\left(\overline{\mathbf{3}}_{A}, \mathbf{1}\right)_{\mathbf{1} / \mathbf{3}, \mathbf{2} / \mathbf{3}}^{(0,0)}+6 \times\left(\mathbf{1}, \mathbf{3}_{S}\right)_{\mathbf{0}, \mathbf{0}}^{(0,0)}+(3-\tilde{x}) \times(\mathbf{1}, \mathbf{1})_{\mathbf{1}, \mathbf{0}}^{(2,0)}+\text { c.c. }\right],
\end{aligned}
$$




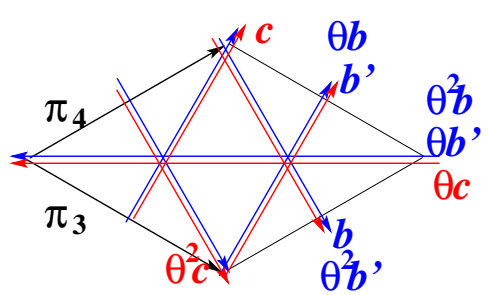

(a)

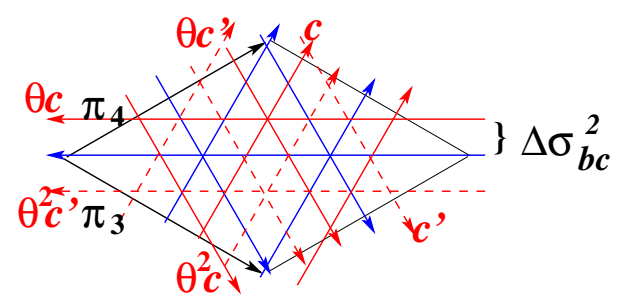

(b)

Figure 9: Gauge symmetry breaking and masses through parallel displacement on $T_{2}^{2}$. (a) branes $b$ and $c$ pass through the origin and carry gauge groups $\mathrm{U}(2)_{b} \times S O / \mathrm{Sp}(2)_{c}$. For brane $c$, the orientifold images are related by $\left(\theta^{k} c\right)=\left(\theta^{k+1} c^{\prime}\right)$. (b) The displacement of brane $c$ breaks $S O / \mathrm{Sp}(2)_{c} \rightarrow \mathrm{U}(1)_{c}$ and renders states at $b\left(\theta^{2} c\right)$ and $b c^{\prime}$ intersections massive.

whereas $\left[V_{H_{1}}\right]$ and $\left[V_{H_{2}}\right]$ are not affected. $S \widehat{O / \mathrm{Sp}}(2)_{\hat{h}_{1}}$ can be broken to $\mathrm{U}(1)_{\hat{h}_{1}}$ via parallel displacements leading to

$$
\begin{aligned}
{\left[V_{H_{3}}\right]^{\left(\Delta \sigma^{2}\right)} } & =9 \times(\mathbf{1}, \mathbf{1})_{\mathbf{0}, \mathbf{0} ; \mathbf{0}}^{(0,0)}+(9-\tilde{y}) \times\left[(\mathbf{1}, \mathbf{1})_{\mathbf{0}, \mathbf{0}, \mathbf{1}}^{(0,0)}+\text { c.c. }\right] \\
& +3 \times\left[(\mathbf{1}, \mathbf{2})_{\mathbf{0}, \mathbf{0} ; \mathbf{1}}^{(0,0)}+(\mathbf{1}, \mathbf{2})_{\mathbf{0}, \mathbf{0} ;-\mathbf{1}}^{(0,0)}+(\mathbf{1}, \mathbf{1})_{\mathbf{1} / \mathbf{2}, \mathbf{0} ; \mathbf{1}}^{(1,0)}+(\mathbf{1}, \mathbf{1})_{\mathbf{1} / \mathbf{2}, \mathbf{0} ;-\mathbf{1}}^{(1,0)}+\text { c.c. }\right]
\end{aligned}
$$

with $0 \leq \tilde{y} \leq 9$.

The beta function coefficients are then lowered to

$$
b_{\mathrm{SU}(3)_{a}}^{\left(\Delta \sigma^{2}\right)}=12, \quad b_{\mathrm{SU}(2)_{b}}^{\left(\Delta \sigma^{2}\right)}=65, \quad b_{\mathrm{U}(1)_{Y}}^{\left(\Delta \sigma^{2}\right)}=42-2 \tilde{x}, \quad b_{\mathrm{U}(1)_{B-L}}^{\left(\Delta \sigma^{2}\right)}=72 .
$$

These string-scale values are clearly not realistic, in particular $b_{\mathrm{SU}(3)_{a}}^{\left(\Delta \sigma^{2}\right)}$ has the wrong sign.

\subsection{A three generation model without hidden sector}

As mentioned in section 6.7, we found 61,440 models without any hidden sector. There are two types of models which can be classified by the number of $h$ Higgs-up and $h$ Higgs-down multiplets (and vector-like lepton pairs) that occur. As shown in equation (6.3), there are two groups with $h=18$ and 21, respectively. In the following we analyse one example of a model with $h=21$ Higgs candidates, or more precisely 18 Higgs generations and three vector-like lepton pairs distinguished by their $B-L$ charge, in more detail.

The model is realised with a value of $\varrho=1 / 4$ for the complex structure parameter on $T_{3}^{2}$. The configuration of bulk branes is given in table 19 and the exceptional part containing the displacements $\sigma_{i}$ and Wilson lines $\tau^{j}$ in table 20.

In tables 21 and 22 we list the intersection numbers leading to the chiral and full matter spectrum, respectively.

Concerning the $\mathrm{U}(1)$ factors, we again find that $\mathrm{U}(1)_{b}$ and the combination $3 \mathrm{U}(1)_{a}-$ $\mathrm{U}(1)_{d}$ are massive, but we have a massless $\mathrm{U}(1)_{B-L}$ symmetry given by $\frac{1}{3} \mathrm{U}(1)_{a}+\mathrm{U}(1)_{d}$. Furthermore brane $c$ leads to an additional massless U(1), or provides a (purposefully broken) $S O / \mathrm{Sp}(2)$ symmetry (brane $c$ corresponds to (1a) in the classification of orientifold 


\begin{tabular}{|c|c|c|c|crr|}
\hline \multicolumn{7}{|c|}{ Ex. w/o hidden sector: bulk brane configuration } \\
\hline brane & $\left(n_{1}, m_{1} ; n_{2}, m_{2} ; n_{3}, m_{3}\right)$ & $N$ & $\tilde{a}_{1} \equiv P, \tilde{a}_{2} \equiv Q, \tilde{a}_{3} \equiv U, \tilde{a}_{4} \equiv V$ \\
\hline$a$ & $(1,-1 ; 1,0 ; 0,1)$ & 3 & 0, & 0, & 1, & -1 \\
\hline$b$ & $(1,1 ; 2,-1 ; 1,2)$ & 2 & 3, & 0, & 6, & 0 \\
\hline$c$ & $(1,-1 ;-1,2 ; 1,0)$ & 1 & 1, & 1, & 0, & 0 \\
\hline$d$ & $(1,1 ; 1,-2 ; 0,1)$ & 1 & 0, & 0, & 3, & -3 \\
\hline
\end{tabular}

Table 19: Bulk brane configuration for a standard model example without hidden sector. Only $m_{3}^{b}$ differs from the bulk configurations with hidden branes in section 7.1 .

\begin{tabular}{|c|cccc|ccccc|ccccc|ccc|}
\hline \multicolumn{10}{|c|}{ Ex. w/o hidden sector: exceptional brane configuration } \\
\hline brane & $d_{1}$, & $d_{2}$, & $d_{3}$, & $d_{4}$ & $e_{1}$, & $e_{2}$, & $e_{3}$, & $e_{4}$ & $\sigma_{1}$, & $\sigma_{2}$, & $\sigma_{5}$, & $\sigma_{6}$ & $\tau^{0}$, & $\tau^{1}$, & $\tau^{3}$ \\
\hline$a$ & 0, & -1, & -1, & 0 & 0, & -1, & -1, & 0 & $\frac{1}{2}$ & 0 & $\frac{1}{2}$ & 0 & 1 & 1 & 0 \\
\hline$b$ & -3, & -3, & 0, & 0 & 0, & 0, & 0, & 0 & $\frac{1}{2}$ & 0 & 0 & 0 & 1 & 1 & 0 \\
\hline$c$ & 3, & 3, & 0, & 0 & -3, & -3, & 0, & 0 & $\frac{1}{2}$ & 0 & 0 & 0 & 1 & 1 & 0 \\
\hline$d$ & 0, & 1, & 1, & 0 & 0, & 1, & 1, & 0 & 0 & 0 & $\frac{1}{2}$ & 0 & 0 & 0 & 0 \\
\hline
\end{tabular}

Table 20: Exceptional brane configuration for a standard model example without hidden sector.

\begin{tabular}{|c|r|r|r|r|r|r|r|r|}
\hline \multicolumn{10}{|c|}{ Ex. w/o hidden sector: chiral matter spectrum } \\
\hline brane & $\chi^{\text {Anti }}$ & $\chi^{\text {Sym }}$ & $\chi^{\cdot \mathbf{b}}$ & $\chi^{\cdot \mathbf{c}}$ & $\chi^{\cdot \mathbf{d}}$ & $\chi^{\cdot \mathbf{b}^{\prime}}$ & $\chi^{\cdot \mathbf{c}^{\prime}}$ & $\chi^{\cdot \mathbf{d}^{\prime}}$ \\
\hline$a$ & 0 & 0 & 0 & $-3\left(d_{R}\right)$ & 0 & $3\left(Q_{L}\right)$ & $-3\left(u_{R}\right)$ & 0 \\
\hline$b$ & -9 & 0 & & $-18\left(H_{u}\right)$ & $6(L)$ & & $-18\left(H_{d}\right)$ & $3(\bar{L})$ \\
\hline$c$ & 0 & 0 & \multicolumn{10}{|c|}{} & $-3\left(\nu_{R}\right)$ & & & $3\left(e_{R}\right)$ \\
\hline$d$ & 0 & 0 & \multicolumn{10}{|c|}{} \\
\hline
\end{tabular}

Table 21: Chiral spectrum of a model without a hidden sector. The identifications with standard model-like particles are given in parenthesis, see also equation (7.13).

\begin{tabular}{|c|r|r|r|r|r|r|r|r|r|}
\hline \multicolumn{1}{|c|}{ Ex. w/o hidden sector: complete matter spectrum } \\
\hline brane & $\varphi^{\text {Adj }}$ & $\varphi^{\text {Anti }}$ & $\varphi^{\text {Sym }}$ & $\varphi^{\cdot \mathbf{b}}$ & $\varphi^{\mathbf{c}}$ & $\varphi^{\cdot \mathbf{d}}$ & $\varphi^{\cdot \mathbf{b}^{\prime}}$ & $\varphi^{\cdot \mathbf{c}^{\prime}}$ & $\varphi^{\cdot \mathbf{d}^{\prime}}$ \\
\hline$a$ & 2 & 6 & 0 & 0 & 3 & 6 & 5 & 3 & 6 \\
\hline$b$ & 10 & 11 & 20 & & 22 & 10 & & 22 & 5 \\
\hline$c$ & 4 & $(2 x)$ & $8-2 x$ & & 5 & & & 5 \\
\hline$d$ & 10 & $(22)$ & 0 & \multicolumn{1}{|c|}{} & \multicolumn{4}{|c}{} \\
\hline
\end{tabular}

Table 22: The complete non-chiral matter spectrum of the standard model example without hidden sector.

invariant branes in [11]). We can thus take $\mathrm{U}(1)_{B-L}$ and $\mathrm{U}(1)_{Y}$ to span the space of massless Abelian gauge factors as in equation (7.1). 
The complete matter spectrum can be divided into a chiral part $[C]$ derived from non-vanishing 3-cycle intersection numbers,

$$
\begin{aligned}
& {[C]=3 \times } {\left[(\mathbf{3}, \mathbf{2})_{\mathbf{1} / \mathbf{6}, \mathbf{1} / \mathbf{3}}^{(0,0)}+(\overline{\mathbf{3}}, \mathbf{1})_{\mathbf{1} / \mathbf{3},-\mathbf{1} / \mathbf{3}}^{(1,0)}+(\overline{\mathbf{3}}, \mathbf{1})_{-\mathbf{2} / \mathbf{3},-\mathbf{1} / \mathbf{3}}^{(-1,)}+(\mathbf{1}, \mathbf{1})_{\mathbf{1}, \mathbf{1}}^{(1,1)}+(\mathbf{1}, \mathbf{1})_{\mathbf{0}, \mathbf{1}}^{(-1,1)}\right.} \\
&+2 \times(\mathbf{1}, \mathbf{2})_{-\mathbf{1} / \mathbf{2},-\mathbf{1}}^{(0,-1)}+(\mathbf{1}, \mathbf{2})_{\mathbf{1} / \mathbf{2}, \mathbf{1}}^{(0,1)}+6 \times(\mathbf{1}, \overline{\mathbf{2}})_{-\mathbf{1} / \mathbf{2}, \mathbf{0}}^{(-1,0)}+6 \times(\mathbf{1}, \overline{\mathbf{2}})_{\mathbf{1} / \mathbf{2}, \mathbf{0}}^{(1,0)} \\
&\left.+3 \times\left(\mathbf{1}, \mathbf{1}_{\bar{A}}\right)_{\mathbf{0}, \mathbf{0}}^{(0,0)}\right] \\
& \equiv 3 \times\left[Q_{L}+d_{R}+u_{R}+e_{R}+\nu_{R}+2 \times L+\bar{L}\right]+18 \times\left[H_{d}+H_{u}\right]+9 \times S
\end{aligned}
$$

and a non-chiral part $[V]$, which consists of matter in the adjoint representation of the gauge group and matter from the $\mathcal{N}=2$ sectors, listed here as chiral representations + c.c. (complex conjugate) inside square brackets.

$$
\begin{aligned}
{[V]=2 \times } & (\mathbf{8}, \mathbf{1})_{\mathbf{0}, \mathbf{0}}^{(0,0)}+10 \times(\mathbf{1}, \mathbf{3})_{\mathbf{0}, \mathbf{0}}^{(0,0)}+26 \times(\mathbf{1}, \mathbf{1})_{\mathbf{0}, \mathbf{0}}^{(0,0)} \\
+ & (\mathbf{3}, \mathbf{2})_{\mathbf{1} / \mathbf{6}, \mathbf{1} / \mathbf{3}}^{(0,0)}+3 \times(\overline{\mathbf{3}}, \mathbf{1})_{\mathbf{1} / \mathbf{3}, \mathbf{2} / \mathbf{3}}^{(0,1)}+3 \times(\overline{\mathbf{3}}, \mathbf{1})_{-\mathbf{2} / \mathbf{3},-\mathbf{4} / \mathbf{3}}^{(0,-1)} \\
& +\left(3-x+1_{m}\right) \times(\mathbf{1}, \mathbf{1})_{\mathbf{1}, \mathbf{0}}^{(2,0)}+\left(1+2{ }_{m}\right) \times\left(\overline{\mathbf{3}}_{A}, \mathbf{1}\right)_{\mathbf{1} / \mathbf{3}, \mathbf{2} / \mathbf{3}}^{(0,0)} \\
& +\left(9+1_{m}\right) \times\left(\mathbf{1}, \mathbf{3}_{S}\right)_{\mathbf{0 , 0}}^{(0,0)}+2_{m} \times(\mathbf{1}, \overline{\mathbf{2}})_{-\mathbf{1} / \mathbf{2}, \mathbf{0}}^{(-1,0)} \\
& +2_{m} \times(\mathbf{1}, \overline{\mathbf{2}})_{\mathbf{1} / \mathbf{2}, \mathbf{0}}^{(1,0)}+2_{m} \times(\mathbf{1}, \mathbf{2})_{-\mathbf{1} / \mathbf{2},-\mathbf{1}}^{(0,-1)}+1_{m} \times(\mathbf{1}, \mathbf{2})_{\mathbf{1} / \mathbf{2}, \mathbf{1}}^{(0,1)} \\
& \left.+1_{m} \times\left(\mathbf{1}, \mathbf{1}_{A}\right)_{\mathbf{0}, \mathbf{0}}^{(0,0)}+1_{m} \times(\mathbf{1}, \mathbf{1})_{\mathbf{0},-\mathbf{1}}^{(1,-1)}+1_{m} \times(\mathbf{1}, \mathbf{1})_{\mathbf{1}, \mathbf{1}}^{(1,1)}+\text { c.c. }\right] .
\end{aligned}
$$

To keep the notation compact, the multiplicities of non-chiral states which arise from sectors with D6-branes parallel along $T_{2}^{2}$ and thus massive when branes are parallely displaced in a continuous manner are labelled by a lower index $m$. The last two lines of $[V]$ disappear completely from the massless spectrum if suitable distances $\Delta \sigma^{2}$ are chosen, and the multiplicities of states in the third and fourth line of $[V]$ are reduced.

All states in the "chiral" sector $[C]$ derive from non-vanishing intersection numbers, but as in the previous examples, since $\mathrm{U}(1)_{b}$ acquires a mass through the Green Schwarz mechanism by absorbing a neutral closed string field, the pairings of Higgs fields, $H_{d}+H_{u}$, and leptons, $L+\bar{L}$, and the states $S \equiv\left(\mathbf{1}, \mathbf{1}_{\bar{A}}\right)_{\mathbf{0 . 0}}^{(0,0)}$ are vector-like with respect to the massless gauge group $\mathrm{SU}(3)_{a} \times \mathrm{SU}(2)_{b} \times \mathrm{U}(1)_{Y} \times \mathrm{U}(1)_{B-L}$ of this model. The selection rules for perturbative superpotential couplings of standard model particles are identical to the previous examples, see equations (7.3) and (7.4), since all quarks, leptons and Higgses arise from the same intersection pattern as displayed in equation (6.3) or by comparison of tables 16 and 21 . 
The beta function coefficients for this model are

$$
\begin{array}{rlc} 
& {[C]+[V],} & {[C]+[V] \text { minus massive }} \\
b_{\mathrm{SU}(3)_{a}}= & 14, & \text { states in }(7.14), \\
b_{\mathrm{SU}(2)_{b}}= & 91, & 12, \\
b_{\mathrm{U}(1)_{Y}}= & \frac{181}{3}-2 x, & 80, \\
b_{\mathrm{U}(1)_{B-L}}= & \frac{280}{3}, & 48-2 x,
\end{array}
$$

Again, in order to obtain realistic values, more non-chiral fields have to acquire a mass which necessitates the exact computation of couplings or $n$-point functions and clearly goes beyond the scope of this paper.

\section{Conclusions}

In this article, we have continued the statistical analysis of supersymmetric intersecting D6-brane models on $T^{6} / \mathbb{Z}_{6}^{\prime}$ with the result that the seemingly large variety of spectra and gauge couplings observed in [11] is subject to very strong correlations as displayed for the number of chiral exotics versus Higgses in table 9. Most of the models without chiral exotics contain nine generations of chiral up- and down-type Higgs multiplets plus three vector-like leptons pairs stemming from non-vanishing 3-cycle intersection numbers in the examples where a $B-L$ symmetry distinguishes Higgses and sleptons. Considering the hidden sectors, only gauge groups of at most rank three occur. All of these models have the same weak mixing angle and ratio of strong and weak gauge couplings at the string scale.

We have thus seen that there exist correlations between certain properties of models with the gauge and chiral matter content of the standard model and an unconstrained hidden sector. Whether this is true in a bigger context or an artefact of the specific geometric set-up of these models is an interesting open question. The correlations between other properties of theses models certainly also deserve further study and should be compared with different constructions. ${ }^{18}$ Unfortunately, a statistical analysis of the complete nonchiral matter spectrum has turned out to be too ambitious due to the numerous distinctions of special cases discussed in section 3.2.

We found that in the examples discussed in detail in section the one-loop beta function coefficient for the strong coupling has the wrong sign, unless most of the nonchiral states acquire masses at a high enough scale. To see if this can occur for states other than those parallel on $T_{2}^{2}$, it will be necessary to determine interaction terms for the fractional branes under consideration. ${ }^{19}$ We hope to come back to this point in future work.

The analyses of the $T^{6} / \mathbb{Z}_{6}$ and $T^{6} / \mathbb{Z}_{6}^{\prime}$ intersecting D6-brane orbifold models suggest that three standard model-like generations are naturally realised in the presence of a $\mathbb{Z}_{3}$ symmetry, which was not expected from the originally proposed configuration in [42], but

\footnotetext{
${ }^{18}$ For some results concerning correlations on $T^{6} /\left(\mathbb{Z}_{2} \times \mathbb{Z}_{2}\right)$ and $T^{6} / \mathbb{Z}_{6}$ see [月, 40, 30].

${ }^{19}$ For toroidal branes, some results have been reported in [41], and for toroidal instanton computations see e.g. 12, 13]. Threshold corrections and Kähler metrics on $T^{6} /\left(\mathbb{Z}_{2} \times \mathbb{Z}_{2}\right)$ with rigid branes have been considered in [37].
} 
occurs also in heterotic orbifold models, see e.g. [1] and references therein. It seems therefore natural to expect phenomenologically interesting models also on, e.g., $T^{6} / \mathbb{Z}_{6} \times \mathbb{Z}_{2}$ orbifold backgrounds with torsion and rigid cycles [30].

\section{Acknowledgments}

G. H. thanks Oleg Lebedev, Fernando Marchesano and Angel Uranga for helpful discussions. The work of F. G. is supported by the Foundation for Fundamental Research of Matter (FOM) and the National Organisation for Scientific Research (NWO).

\section{A. Chan-Paton labels}

The representations of four dimensional massless open string states in a $T^{6} / \mathbb{Z}_{2 N}$ background depend on the $\mathbb{Z}_{2} \equiv \theta^{N}$ orbifold and $\Omega \mathcal{R} \theta^{-k}$ orientifold projections on the ChanPaton labels $\lambda$,

$$
\begin{aligned}
& \lambda=a_{\mathbb{Z}_{2}}\left(\gamma_{\mathbb{Z}_{2}} \lambda \gamma_{\mathbb{Z}_{2}}^{-1}\right), \\
& \lambda=a_{\Omega \mathcal{R} \theta^{-k}}\left(\gamma_{\Omega \mathcal{R} \theta^{-k}} \lambda \gamma_{\Omega \mathcal{R} \theta^{-k}}^{-1}\right)^{T}, \\
& \lambda=a_{\Omega \mathcal{R} \theta^{-k+N}}\left(\gamma_{\Omega \mathcal{R} \theta^{-k+N}} \lambda \gamma_{\Omega \mathcal{R} \theta^{-k+N}}^{-1}\right)^{T},
\end{aligned}
$$

with the eigenvalues $a_{\mathbb{Z}_{2}}, a_{\Omega \mathcal{R} \theta^{-k}}= \pm 1$ of massless states and the consistency conditions on the combined orbifold and orientifold action

$$
a_{\Omega \mathcal{R} \theta^{-k+N}}=a_{\mathbb{Z}_{2}} \cdot a_{\Omega \mathcal{R} \theta^{-k}}, \quad \gamma_{\Omega \mathcal{R} \theta^{-k+N}}=\gamma_{\Omega \mathcal{R} \theta^{-k}} \cdot \gamma_{\mathbb{Z}_{2}}
$$

The transformation properties of the intersection points determine which projection conditions have to be imposed on the Chan-Paton matrices $\lambda$. (3.15) applies to intersection points which are neither $\mathbb{Z}_{2}$ nor $\Omega \mathcal{R} \theta^{-k}$ or $\Omega \mathcal{R} \theta^{-k+N}$ invariant, which corresponds to the notation $\left(a_{\mathbb{Z}_{2}}, a_{\Omega \mathcal{R} \theta^{-k}}, a_{\Omega \mathcal{R} \theta^{-k+N}}\right)=(*, *, *)$. The remaining cases are listed in table 23 .

\section{B. Massless open string states}

The general mass formula for states localised at brane intersections reads

$$
\frac{\alpha^{\prime}}{4} m^{2}=\frac{1}{2} p^{2}+E_{T}-\frac{1}{2} \quad \text { with } \quad E_{T}=\frac{1}{2} \sum_{i=1}^{3} \phi_{i}\left(1-\phi_{i}\right) \quad \text { and } \quad \phi_{i} \in[0,1),
$$

where $\pi \phi_{i}$ is the angle between the branes on $T_{i}^{2}$ up to a possible shift of $\pi$.

Setting $\phi_{0} \equiv 0$, in the NS sector the states at the brane intersection take the form

$$
p=|\vec{n}-\vec{\phi}\rangle
$$

with $n_{i} \in \mathbb{Z}$, and the raising and lowering oscillators are $\psi_{-1 / 2+m}^{\mu}, \psi_{-1 / 2+\phi_{i}+m}^{i}, \psi_{-1 / 2-\phi_{i}+m}^{\bar{i}}$, with $i=1,2,3$ and $m \in \mathbb{Z}$. GSO invariant states comply with $\sum_{i} n_{i}=$ odd. 


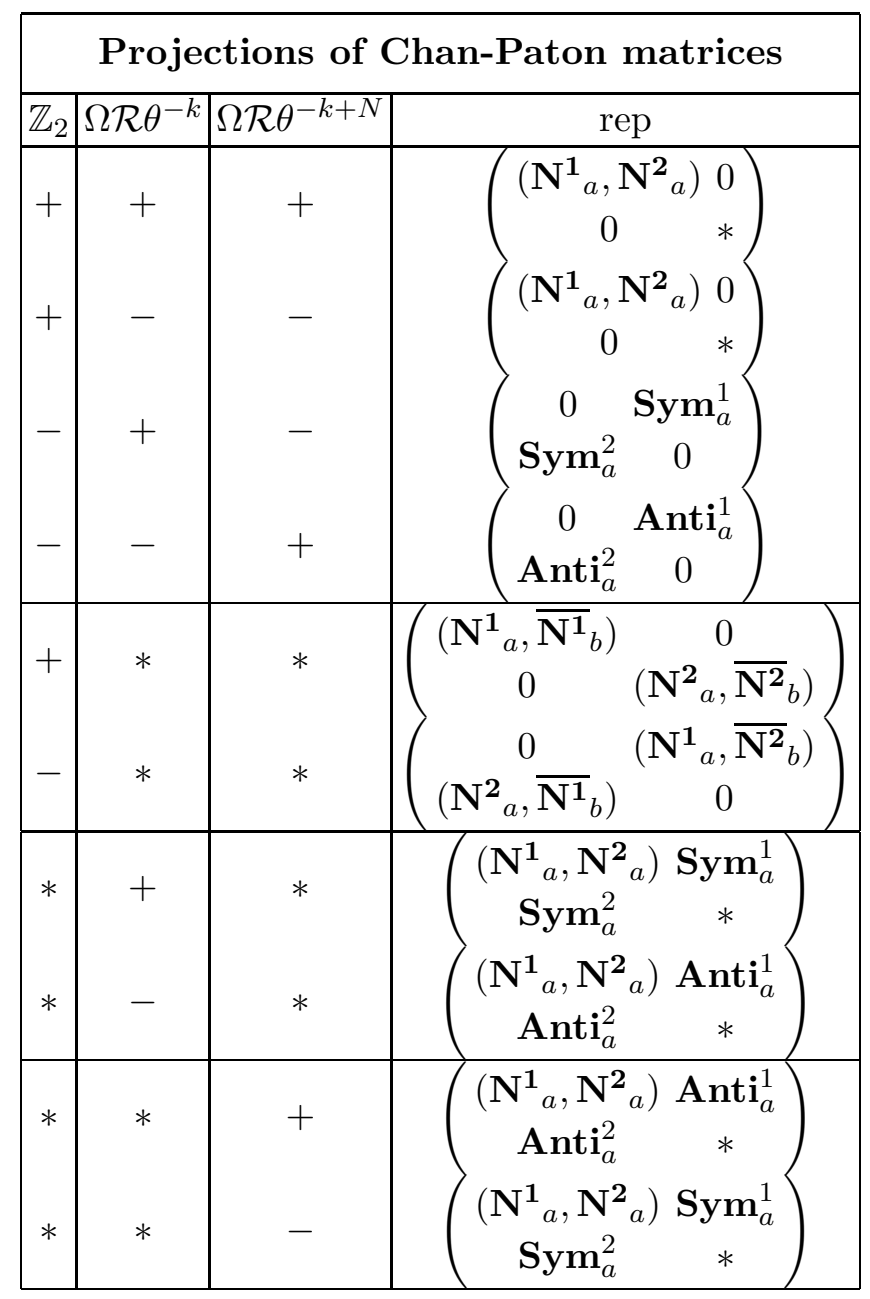

Table 23: Projections of Chan-Paton labels for various combinations of orbifold and orientifold eigenvalues $\left(a_{\mathbb{Z}_{2}}, a_{\Omega \mathcal{R} \theta^{-k}}, a_{\Omega \mathcal{R} \theta^{-k+N}}\right)$. The entry $*$ for an eigenvalue denotes no projection condition. A star in the lower right entry of the Chan-Paton label appears whenever this entry is by the orientifold projection related to the upper left entry thereby not providing any new degrees of freedom (d.o.f.).

The $\mathrm{R}$ sector states at brane intersections are given by

$$
p=\left|\vec{n}+\frac{\overrightarrow{1}}{2}-\vec{\phi}\right\rangle
$$

with corresponding oscillator states $\psi_{m}^{0}, \psi_{\phi_{i}+m}^{i}, \psi_{-\phi_{i}+m}^{\bar{i}}$, where $\psi_{0}^{0}$ flips the four dimensional chirality of a given state. We use the convention that the GSO projection enforces $\sum_{i} n_{i}=$ even.

The resulting massless and GSO projected open string states for supersymmetric configurations of D6-branes at angles are given in table 24. Generically, the state $a\left(\theta^{k} b\right)$ at a supersymmetric brane intersection with angles $\pi\left(\phi_{1}, \phi_{2}, \phi_{3}\right)$ and $\sum_{i=1}^{3} \phi_{i}=0 \bmod 2$ provides one massless NS and R sector d.o.f. with a given $\mathbb{Z}_{2}$ parity. Its inverse sector $\left(\theta^{k} b\right) a$ 


\begin{tabular}{|c|c|c|c|}
\hline \multicolumn{4}{|c|}{ Massless open string states } \\
\hline$\left(\phi_{1}, \phi_{2}, \phi_{3}\right)$ & NS & $\mathrm{R}$ & $\tilde{a}_{\mathbb{Z}_{2}}$ \\
\hline$(0,0,0)$ & $\begin{array}{l}| \pm 1,0,0,0\rangle_{\mathrm{NS}}=\psi_{-1 / 2}^{\mu}|0\rangle_{\mathrm{NS}} \\
|0, \pm 1,0,0\rangle_{\mathrm{NS}}=\psi_{-1 / 2}^{1, \overline{1}}|0\rangle_{\mathrm{NS}} \\
|0,0, \pm 1,0\rangle_{\mathrm{NS}}=\psi_{-1 / 2}^{2, \overline{2}}|0\rangle_{\mathrm{NS}} \\
|0,0,0, \pm 1\rangle_{\mathrm{NS}}=\psi_{-1 / 2}^{3, \frac{3}{3}}|0\rangle_{\mathrm{NS}}\end{array}$ & $\begin{array}{c} \pm\left|\frac{1}{2}, \frac{1}{2}, \frac{1}{2}, \frac{1}{2}\right\rangle_{\mathrm{R}}=|0\rangle_{\mathrm{R}},\left(\prod_{i=0}^{3} \psi_{0}^{i}\right)|0\rangle_{\mathrm{R}} \\
\psi_{0}^{0} \psi_{0}^{1}|0\rangle_{\mathrm{R}}, \psi_{0}^{2} \psi_{0}^{3}|0\rangle_{\mathrm{R}} \\
\psi_{0}^{0} \psi_{0}^{2}|0\rangle_{\mathrm{R}}, \psi_{0}^{1} \psi_{0}^{3}|0\rangle_{\mathrm{R}} \\
\psi_{0}^{0} \psi_{0}^{3}|0\rangle_{\mathrm{R}}, \psi_{0}^{1} \psi_{0}^{2}|0\rangle_{\mathrm{R}} \\
\end{array}$ & $\begin{array}{l}+ \\
- \\
+ \\
-\end{array}$ \\
\hline $\begin{array}{l}(\phi,-\phi, 0) \\
(\phi, 0,-\phi) \\
(0, \phi,-\phi)\end{array}$ & $\begin{array}{c}|0,-\phi, \phi-1,0\rangle_{\mathrm{NS}}=\psi_{-1 / 2+\phi}^{2}|0\rangle_{\mathrm{NS}} \\
|0,1-\phi, \phi, 0\rangle_{\mathrm{NS}}=\psi_{-1 / 2+\phi}^{1}|0\rangle_{\mathrm{NS}} \\
|0,-\phi, 0, \phi-1\rangle_{\mathrm{NS}}=\psi_{-1 / 2+\phi}^{3}|0\rangle_{\mathrm{NS}} \\
|0,1-\phi, 0, \phi\rangle_{\mathrm{NS}}=\psi_{-1 / 2+\phi}^{\overline{1}}|0\rangle_{\mathrm{NS}} \\
|0,0,-\phi, \phi-1\rangle_{\mathrm{NS}}=\psi_{-1 / 2+\phi}^{3}|0\rangle_{\mathrm{NS}} \\
|0,0,1-\phi, \phi\rangle_{\mathrm{NS}}=\psi_{-1 / 2+\phi}^{2}|0\rangle_{\mathrm{NS}}\end{array}$ & 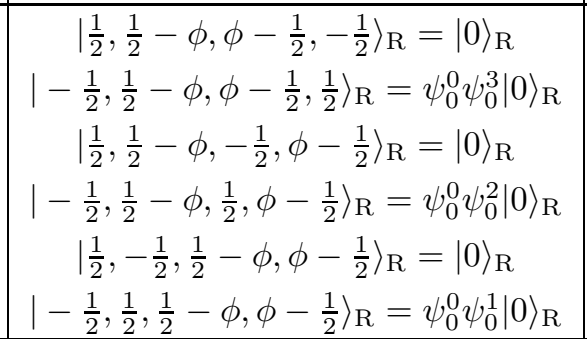 & $\begin{array}{l}+ \\
- \\
- \\
- \\
- \\
+\end{array}$ \\
\hline $\begin{array}{c}\left(\phi_{1}, \phi_{2},-\phi_{1}-\phi_{2}\right) \\
\text { for } 0<\phi_{1}+\phi_{2}<1 \\
\text { for } 1<\phi_{1}+\phi_{2}<2\end{array}$ & $\begin{array}{c}\left|0,-\phi_{1},-\phi_{2}, \phi_{1}+\phi_{2}-1\right\rangle_{\mathrm{NS}} \\
\left|0,1-\phi_{1}, 1-\phi_{2}, \phi_{1}+\phi_{2}-1\right\rangle_{\mathrm{NS}}\end{array}$ & $\begin{array}{c}\left|-\frac{1}{2}, \frac{1}{2}-\phi_{1}, \frac{1}{2}-\phi_{2}, \phi_{1}+\phi_{2}-\frac{1}{2}\right\rangle_{\mathrm{R}} \\
\left|\frac{1}{2}, \frac{1}{2}-\phi_{1}, \frac{1}{2}-\phi_{2}, \phi_{1}+\phi_{2}-\frac{3}{2}\right\rangle_{\mathrm{R}}\end{array}$ & $\begin{array}{l}+ \\
+\end{array}$ \\
\hline
\end{tabular}

Table 24: GSO projected massless NS and R states for supersymmetric configurations of D6-branes at angles. The $\mathbb{Z}_{2}$ subgroup of $T^{6} / \mathbb{Z}_{2 N}$ is given by $N \vec{v}=\left(\frac{1}{2}, 0,-\frac{1}{2}\right)$. For shortness, $\pm\left|\frac{1}{2}, \frac{1}{2}, \frac{1}{2}, \frac{1}{2}\right\rangle_{\mathrm{R}}$ denotes the two states $\left|\frac{1}{2}, \frac{1}{2}, \frac{1}{2}, \frac{1}{2}\right\rangle_{\mathrm{R}}$ and $\left|-\frac{1}{2},-\frac{1}{2},-\frac{1}{2},-\frac{1}{2}\right\rangle_{\mathrm{R}}$. The parameter range is chosen to be $0<\phi, \phi_{1}, \phi_{2}<1$. In the absence of Wilson lines, the sign $\tilde{a}_{\mathbb{Z}_{2}}$ coincides with the one occurring in the projection of the Chan-Paton labels, $a_{\mathbb{Z}_{2}}$.

at angles $\pi\left(-\phi_{1},-\phi_{2},-\phi_{3}\right)$ furnishes the missing d.o.f. to form a full chiral multiplet. The $\mathbb{Z}_{2}$ parity is read off from the fact that the unique (tachyonic) NS ground-states $|0\rangle_{\mathrm{NS}}$ is $\mathbb{Z}_{2}$ even, while in the sector with one vanishing angle, e.g. $\pi(\phi,-\phi, 0)$, the oscillator $\psi_{-1 / 2+\phi}^{2}$ is also $\mathbb{Z}_{2}$ even, but $\psi_{-1 / 2+\phi}^{\overline{1}}$ is $\mathbb{Z}_{2}$ odd.

\section{Matter on $\operatorname{SO}(2 N) / \operatorname{Sp}(2 N)$ branes}

In 11], we (partially, see footnote in section 7.1) classified branes which carry $\mathrm{SO}(2 N)$ or $\mathrm{Sp}(2 N)$ gauge factors. Independently of deriving the correct gauge group assignment, it is possible to compute the number of symmetric plus antisymmetric matter states as follows. The $\gamma_{\mathbb{Z}_{2}}$ matrix is the same as in equation (3.13), but the $\Omega \mathcal{R} \theta^{-k}$ projection and thereby the identification of representations in the Chan-Paton labels for a $\Omega \mathcal{R}$ invariant brane $c$ changes,

$$
\gamma_{\Omega \mathcal{R} \theta^{-k}}=\mathbb{I}, \quad \lambda_{c\left(\theta^{k} c\right)} \simeq\left(\begin{array}{cc}
\mathbf{A n t i}_{c_{1}}+\mathbf{S y m}_{c_{1}} & \left(\mathbf{2 N}_{c_{1}}, \mathbf{2 N}_{c_{2}}\right) \\
\left(\mathbf{2 N}_{c_{1}}, \mathbf{2} \mathbf{N}_{c_{2}}\right) & \mathbf{A n t i}_{c_{2}}+\mathbf{S y m}_{c_{2}}
\end{array}\right),
$$

where as before we include both fractional branes $c_{1}+c_{2}=c$ which are individually $\Omega \mathcal{R}$ invariant and have opposite $\mathbb{Z}_{2}$ eigenvalue and vanishing relative Wilson lines.

The matter states and their $\mathbb{Z}_{2}$ eigenvalues are read off from table 24 leading to one multiplet in the adjoint representation plus two multiplets transforming as $\left(\mathbf{2 N}_{c_{1}}, \mathbf{2} \mathbf{N}_{c_{2}}\right)$ 
in the $c c$ sector. The $c\left(\theta^{k} c\right)$ sectors for $k=1,2$ have angles $\pi\left( \pm \frac{1}{3}, \mp \frac{1}{3}, 0\right)$, and the corresponding representations depend on the invariance properties of the intersection points and the (undetermined) $\Omega \mathcal{R} \theta^{-k}$ eigenvalue of the massless state,

- $z_{\Omega \mathcal{R} \theta^{-k}+\mathbb{Z}_{2}}: \Omega \mathcal{R} \theta^{-k}$ and $\mathbb{Z}_{2}$ invariant points on $T_{1}^{2} \times T_{2}^{2}$ :

$$
\left(\mathbf{2 N}_{c_{1}}, \mathbf{2 N}_{c_{2}}\right)+\left\{\begin{array}{l}
\Omega \mathcal{R} \theta^{-k}:+\mathbf{S y m}_{c_{1}}+\mathbf{S y m}_{c_{2}} \\
\Omega \mathcal{R} \theta^{-k}:-\mathbf{A n t i}_{c_{1}}+\mathbf{A n t i}_{c_{2}}
\end{array}\right.
$$

- $z_{\Omega \mathcal{R} \theta^{-k(+N)}}: \Omega \mathcal{R} \theta^{-k(+N)}$ but not $\mathbb{Z}_{2}$ invariant orbits on $T_{1}^{2} \times T_{2}^{2}$ :

$$
2 \times\left(\mathbf{2 N}_{c_{1}}, \mathbf{2 N}_{c_{2}}\right)+2 \times\left\{\begin{array}{l}
\Omega \mathcal{R} \theta^{-k(+N)}:+\mathbf{S y m}_{c_{1}}+\mathbf{S y m}_{c_{2}} \\
\Omega \mathcal{R} \theta^{-k(+N)}:-\mathbf{A n t i}_{c_{1}}+\mathbf{A n t i}_{c_{2}}
\end{array},\right.
$$

- $z_{\mathbb{Z}_{2}}: \mathbb{Z}_{2}$ but not $\Omega \mathcal{R} \theta^{-k}$ invariant orbits:

$$
2 \times\left(\mathbf{2 N}_{c_{1}}, \mathbf{2 N}_{c_{2}}\right)+\mathbf{S y m}_{c_{1}}+\mathbf{S y m}_{c_{2}}+\mathbf{A n t i}_{c_{1}}+\mathbf{A n t i}_{c_{2}}
$$

- $z_{0}$ : orbits of points without invariance properties:

$$
4 \times\left(\mathbf{2 N}_{c_{1}}, \mathbf{2 N}_{c_{2}}\right)+2 \times\left[\mathbf{S y m}_{c_{1}}+\mathbf{S y m}_{c_{2}}+\mathbf{A n t i}_{c_{1}}+\mathbf{A n t i}_{c_{2}}\right] .
$$

Independently of the correct $\Omega \mathcal{R} \theta^{-k}$ assignments, the matter spectrum contains $(i=1,2)$

$$
\begin{aligned}
\#\left(\mathbf{S y m}_{c_{i}}\right)+\#\left(\mathbf{A n t i}_{c_{i}}\right) & =1+\left|I_{c_{i}\left(\theta c_{i}\right)}\right| \\
\#\left(\mathbf{2 N}_{c_{1}}, \mathbf{2 N}_{c_{2}}\right) & =2+\left|I_{c_{1}\left(\theta c_{2}\right)}\right|
\end{aligned}
$$

All other bifundamental representations are counted in the usual way (up to the caveat that $a\left(\theta^{k} c^{\prime}\right)$ sectors are identified with $a\left(\theta^{k} c\right)$ sectors).

\section{Bulk relations among different lattices}

The bulk wrapping numbers $\tilde{a}_{1} \ldots \tilde{a}_{4}$ (labelled $P \ldots V$ in [11) in table 1 can be expressed in terms of one-cycle wrapping numbers $\left(n_{i}, m_{i}\right)_{i=1 \ldots 3}$ on $T_{i}^{2}$ as follows

$$
\tilde{a}_{1} \equiv P=A n_{3}, \quad \tilde{a}_{2} \equiv Q=B n_{3}, \quad \tilde{a}_{3} \equiv U=A m_{3}, \quad \tilde{a}_{4} \equiv V=B m_{3},
$$

with the abbreviations

$$
A \equiv n_{1} n_{2}-m_{1} m_{2}, \quad B \equiv n_{1} m_{2}+m_{1} n_{2}+m_{1} m_{2}
$$

A rotation of $\pi(1 / 3,0,-1 / 2)$ for $b=0$ acts on the one-cycle wrapping numbers as

$$
\left(\begin{array}{ll}
n_{1} & m_{1} \\
n_{2} & m_{2} \\
n_{3} & m_{3}
\end{array}\right) \rightarrow\left(\begin{array}{cc}
-m_{1} & n_{1}+m_{1} \\
n_{2} & m_{2} \\
m_{3} & -n_{3}
\end{array}\right), \quad\left(\begin{array}{c}
A \\
B
\end{array}\right) \rightarrow\left(\begin{array}{c}
-B \\
A+B
\end{array}\right)
$$


The bulk wrapping numbers are thus transformed,

$$
\left(\tilde{a}_{1}, \tilde{a}_{2}, \tilde{a}_{3}, \tilde{a}_{4}\right) \rightarrow\left(-\tilde{a}_{4}, \tilde{a}_{3}+\tilde{a}_{4}, \tilde{a}_{2},-\tilde{a}_{1}-\tilde{a}_{2}\right) \equiv\left(\hat{a}_{1}, \hat{a}_{2}, \hat{a}_{3}, \hat{a}_{4}\right) .
$$

This transformation translates the bulk RR tadpole cancellation condition (2.4) of the AAa geometry to the $\mathbf{B A a}$ lattice and the $\mathbf{A B a}$ to the $\mathbf{B B a}$ constraint.

If the complex structure parameter $\varrho$ on the original torus is replaced by $\hat{\varrho}=\frac{3}{4 \varrho}$, also the bulk supersymmetry conditions (2.5) are reinterpreted in terms of the transformed geometry.

The $\mathbf{b}$ type orientations are included by replacing $\left(n_{3}, m_{3}\right) \rightarrow\left(n_{3}, m_{3}+b n_{3}\right)$ in (D.3) and (D.4).

In [1], a double counting of solutions was avoided as follows.

(i) $\left(n_{1}, m_{1}\right)=($ odd, odd $)$ selects one of the orbifold image cycles $\left(\theta^{k} a\right)$,

(ii) $\left(n_{3}, m_{3}+b n_{3}\right)>(0,0)$ avoids counting the orbit $\left(\theta^{k} a\right)$ and its orientifold image $\left(\theta^{l} a^{\prime}\right)$ as two independent configurations,

(iii) $n_{1}>0$ forbids a simultaneous flip of the remaining orientations of one-cycles along $T_{1}^{2} \times T_{2}^{2}$.

A careful analysis of the solutions related by a $\pi(1 / 3,0,-1 / 2)$ rotation reveals that conditions (i) and (ii) are still met if the $\left(\theta \hat{a}^{\prime}\right)$ image of $a$ is chosen, which has wrapping numbers

$$
\mathbf{B A a}:\left(\hat{n}_{i}, \hat{m}_{i}\right)_{\left(\theta \hat{a}^{\prime}\right)}=\left(\begin{array}{cc}
m_{1} & n_{1} \\
n_{2} & -\left(n_{2}+m_{2}\right) \\
m_{3} & n_{3}
\end{array}\right), \quad \mathbf{B B a}:\left(\begin{array}{cc}
m_{1} & n_{1} \\
n_{2}+m_{2} & -m_{2} \\
m_{3} & n_{3}
\end{array}\right) .
$$

Condition (iii) is replaced by $\hat{m}_{1}>0$. The coprime condition on $T_{1}^{2}$ and $T_{3}^{2}$ is clearly preserved under the transformation, and on $T_{2}^{2}$, it follows from the fact that a $\mathbb{Z}_{6}^{\prime}$ rotation $\theta$ which acts by $\left(n_{2}, m_{2}\right) \stackrel{\theta}{\rightarrow}\left(-n_{2}-m_{2}, n_{2}\right) \stackrel{\theta}{\rightarrow}\left(m_{2},-n_{2}-m_{2}\right)$ also conserves relative primes.

This proves that the number of supersymmetric solutions to the bulk RR tadpole cancellation conditions on AA and $\mathbf{B A}$ geometries along $T_{1}^{2} \times T_{2}^{2}$ is identical, similarly for $\mathbf{A B}$ and $\mathbf{B B}$.

Due to condition (i), the torus cycle is either passing through the $\mathbb{Z}_{2}$ fixed points $\{1,6\}$ or $\{4,5\}$ on $T_{1}^{2}$ in the notation of [11] from which one derives the coefficients $d_{j}, e_{j}$ of the exceptional cycles in (2.3). For some fixed $j_{1}$ which the torus cycle on $T_{3}^{2}$ passes through, one obtains

$$
\begin{aligned}
& \left(d_{j_{1}}, e_{j_{1}}\right)_{\{1,6\}}=\left((-1)^{\tau^{0}+\tau^{1}+1}\left(n_{2}+m_{2}\right),(-1)^{\tau^{0}+\tau^{1}} n_{2}\right), \\
& \left(d_{j_{1}}, e_{j_{1}}\right)_{\{4,5\}}=\left((-1)^{\tau^{0}}\left(n_{2}+(-1)^{\tau^{1}} m_{2}\right),(-1)^{\tau^{0}}\left((-1)^{\tau^{1}+1} n_{2}+\left(1-(-1)^{\tau^{1}}\right) m_{2}\right)\right),
\end{aligned}
$$

where $\left(j_{1}, j_{2}\right) \in\{(1,2),(4,3)\}$ or $\{(1,3),(2,4)\}$ or $\{(1,4),(2,3)\}$ are the pairs of fixed points traversed by the cycle for $\left(n_{3}, m_{3}\right)=$ (odd,even), (odd,odd) and (even, odd), respectively, 
according to the fixed point labels in [11]. The first and second set of points corresponds to no or some displacement of the cycle from the origin on $T_{3}^{2}$, respectively. The coefficients $\left(d_{j_{2}}, e_{j_{2}}\right)$ carry and additional global factor $(-1)^{\tau^{3}}$ where $\tau^{i} \in\{0,1\}$ parameterise the overall $\mathbb{Z}_{2}$ eigenvalue $(i=0)$ and Wilson lines on $T_{1}^{2} \times T_{3}^{2}(i=1,3)$.

Upon the $\pi(1 / 3,0,-1 / 2)$ rotation, the wrapping numbers on $T_{3}^{2}$ transform as $\left(n_{3}, m_{3}\right) \rightarrow\left(m_{3}, n_{3}\right)$ and $\mathbb{Z}_{2}$ fixed points on the a geometry for $T_{3}^{2}$ are permuted as follows,

$$
(1,2,3,4) \rightarrow(1,4,3,2)
$$

Inserting the transformed wrapping numbers $\left(\hat{n}_{2}, \hat{m}_{2}\right)_{\left(\theta \hat{a}^{\prime}\right)}$ on $T_{2}^{2}$ given in (D.5) leads to

BAa :

$$
\begin{aligned}
& \left(\hat{d}_{\hat{j}_{1}}, \hat{e}_{\hat{j}_{1}}\right)_{\{1,6\}}=\left((-1)^{\tau^{0}+\tau^{1}} m_{2},(-1)^{\tau^{0}+\tau^{1}} n_{2}\right), \\
& \left(\hat{d}_{\hat{j}_{1}}, \hat{e}_{\hat{j}_{1}}\right)_{\{4,5\}}=\left((-1)^{\tau^{0}}\left(\left(1-(-1)^{\tau^{1}}\right) n_{2}+(-1)^{\tau^{1}+1} m_{2}\right),(-1)^{\tau^{0}+1}\left(n_{2}+\left(1+(-1)^{\tau^{1}+1}\right) m_{2}\right)\right),
\end{aligned}
$$

BBa :

$$
\begin{aligned}
& \left(\hat{d}_{\hat{j}_{1}}, \hat{e}_{\hat{j}_{1}}\right)_{\{1,6\}}=\left((-1)^{\tau^{0}+\tau^{1}+1} n_{2},(-1)^{\tau^{0}+\tau^{1}}\left(n_{2}+m_{2}\right)\right), \\
& \left(\hat{d}_{\hat{j}_{1}}, \hat{e}_{\hat{j}_{1}}\right)_{\{4,5\}}=\left((-1)^{\tau^{0}}\left(n_{2}+\left(1+(-1)^{\tau^{1}+1}\right) m_{2}\right),(-1)^{\tau^{0}+1}\left((-1)^{\tau^{1}} n_{2}+m_{2}\right)\right),
\end{aligned}
$$

in terms of the original numbers $\left(n_{2}, m_{2}\right)$ on $\mathbf{A A a}$ and $\mathbf{A B a}$, respectively, and the analogous expressions with an overall prefactor of $(-1)^{\tau^{3}}$ for $\left(\hat{d}_{\hat{j}_{2}}, \hat{e}_{\hat{j}_{2}}\right)$.

The RR tadpole cancellation (2.4) and massless hyper charge (5.2) conditions due to exceptional cycles are conveniently parameterised for the a geometry on $T_{3}^{2}$ by $\left(X_{3} \ldots X_{6}\right)$ and $\left(Y_{3} \ldots Y_{6}\right)$ with

$$
X_{2+j}=\left\{\begin{array}{cc}
2 e_{j} & \mathbf{A A a} \\
e_{j}-d_{j} & \mathbf{A B a} \\
d_{j}+e_{j} & \mathbf{B A a} \\
d_{j}+2 e_{j} & \mathbf{B B a}
\end{array}, \quad Y_{2+j}=\left\{\begin{array}{c}
2 d_{j}+e_{j} \\
d_{j}+e_{j} \\
d_{j}-e_{j} \\
2 e_{j}
\end{array} \text { for } \quad j=1 \ldots 4\right.\right.
$$

In case of a $\mathbf{b}$ torus, $X_{3}$ and $X_{6}$ remain unchanged while the remaining two entries are subject to a shift

$$
\left(\begin{array}{l}
X_{4} \\
X_{5}
\end{array}\right) \rightarrow\left(\begin{array}{l}
X_{4}+2 b\left(e_{3}-e_{2}\right) \\
X_{5}-2 b\left(e_{3}-e_{2}\right)
\end{array}\right)
$$

and a similar expression for $\left(Y_{4}, Y_{5}\right)$. This explicit form can be used to test the behaviour of the RR tadpole cancellation conditions from exceptional cycles under the $\pi(1 / 3,0,-1 / 2)$ rotation. The correspondence of the bulk relations does, however, not carry over immediately. This is not too surprising since,e.g., the number of full solutions on $\mathbf{B B a}$ is by one order of magnitude bigger than on ABa [11. 


\begin{tabular}{|c|c|c|}
\hline \multicolumn{3}{|c|}{ Trinification models with $\mathrm{U}(3)_{a} \times \mathrm{U}(3)_{b} \times \mathrm{U}(3)_{c}$} \\
\hline$\underline{Q}$ & chiral states on $a, b, c$ only & \#models \\
\hline$\frac{1}{3}$ & $\begin{array}{c}2 \times\left[\left(\overline{\mathbf{3}}_{a}, \mathbf{3}_{b}, \mathbf{1}\right)+\left(\mathbf{1}, \overline{\mathbf{3}}_{b}, \mathbf{3}_{c}\right)+\left(\mathbf{3}_{a}, \mathbf{1}, \overline{\mathbf{3}}_{c}\right)\right] \\
\quad+6\left(\mathbf{3}_{\bar{A}_{a}}, \mathbf{1}, \mathbf{1}\right)+3\left(\mathbf{6}_{\bar{S}_{a}}, \mathbf{1}, \mathbf{1}\right) \\
+4\left(\mathbf{3}_{a}, \mathbf{3}_{b}, \mathbf{1}\right)+2\left(\mathbf{3}_{a}, \mathbf{1}, \mathbf{3}_{c}\right)+2\left(\mathbf{1}, \overline{\mathbf{3}}_{b}, \overline{\mathbf{3}}_{c}\right)\end{array}$ & 2864 \\
\hline 1 & $\begin{array}{l}2 \times\left[\left(\overline{\mathbf{3}}_{a}, \mathbf{3}_{b}, \mathbf{1}\right)+\left(\mathbf{1}, \overline{\mathbf{3}}_{b}, \mathbf{3}_{c}\right)+\left(\mathbf{3}_{a}, \mathbf{1}, \overline{\mathbf{3}}_{c}\right)\right] \\
\quad+\left(\mathbf{3}_{\bar{A}_{a}}, \mathbf{1}, \mathbf{1}\right)+4\left(\mathbf{6}_{\bar{S}_{a}}, \mathbf{1}, \mathbf{1}\right) \\
+4\left(\mathbf{3}_{a}, \mathbf{3}_{b}, \mathbf{1}\right)+2\left(\mathbf{3}_{a}, \mathbf{1}, \mathbf{3}_{c}\right)+2\left(\mathbf{1}, \overline{\mathbf{3}}_{b}, \overline{\mathbf{3}}_{c}\right)\end{array}$ & $6.3 \times 10^{8}$ \\
\hline
\end{tabular}

Table 25: Complex structure values $\varrho$ on $T_{3}^{2}$, frequency and chiral matter charged under $\mathrm{U}(3)_{a} \times$ $\mathrm{U}(3)_{b} \times \mathrm{U}(3)_{c}$ for trinification models. Further chiral states can arise from intersections with other branes needed for RR tadpole cancellation.

\section{E. Trinification models}

Intersecting D-branes offer the possibility for trinification models with gauge group $\mathrm{U}(3)_{a} \times$ $\mathrm{U}(3)_{b} \times \mathrm{U}(3)_{c}$ and $n$ quark-lepton generations in

$$
n \times\left[\left(\overline{\mathbf{3}}_{a}, \mathbf{3}_{b}, \mathbf{1}\right)+\left(\mathbf{1}, \overline{\mathbf{3}}_{b}, \mathbf{3}_{c}\right)+\left(\mathbf{3}_{a}, \mathbf{1}, \overline{\mathbf{3}}_{c}\right)\right] .
$$

It turns out that on $T^{6} / \mathbb{Z}_{6}^{\prime}$, there are only supersymmetric solutions for $n=2$, and all of them contain a large number of chiral exotics. The chiral matter states involving only stacks $a, b$ and $c$ and the associated complex structure value are displayed in table 25 . More chiral exotic states can arise at intersections with further brane stacks required for $\mathrm{RR}$ tadpole cancellation.

Due to the large number of chiral exotics these models are not very interesting from a phenomenological point of view and we do not pursue a more detailed analysis.

\section{References}

[1] O. Lebedev et al., A mini-landscape of exact MSSM spectra in heterotic orbifolds, Phys. Lett. B 645 (2007) 88 hep-th/0611095.

[2] K.R. Dienes, Statistics on the heterotic landscape: gauge groups and cosmological constants of four-dimensional heterotic strings, Phys. Rev. D 73 (2006) 106010 hep-th/0602286.

[3] P. Anastasopoulos, T.P.T. Dijkstra, E. Kiritsis and A.N. Schellekens, Orientifolds, hypercharge embeddings and the standard model, Nucl. Phys. B 759 (2006) 83 hep-th/0605226.

[4] R. Blumenhagen, F. Gmeiner, G. Honecker, D. Lüst and T. Weigand, The statistics of supersymmetric D-brane models, Nucl. Phys. B 713 (2005) 83 hep-th/0411173.

[5] F. Gmeiner, R. Blumenhagen, G. Honecker, D. Lüst and T. Weigand, One in a billion: MSSM-like D-brane statistics, JHEP 01 (2006) 004 hep-th/0510170. 
[6] F. Gmeiner, Standard model statistics of a type-II orientifold, Fortschr. Phys. 54 (2006) 391 hep-th/0512190.

[7] M.R. Douglas and W. Taylor, The landscape of intersecting brane models, JHEP 01 (2007) 031 hep-th/0606109.

[8] F. Gmeiner and M. Stein, Statistics of SU(5) D-brane models on a type-II orientifold, Phys. Rev. D 73 (2006) 126008 hep-th/0603019.

[9] F. Gmeiner, Gauge sector statistics of intersecting D-brane models, Fortschr. Phys. 55 (2007) 111 hep-th/0608227.

[10] F. Gmeiner, D. Lüst and M. Stein, Statistics of intersecting D-brane models on $T^{6} / Z_{6}$, JHEP 05 (2007) 018 hep-th/0703011.

[11] F. Gmeiner and G. Honecker, Mapping an island in the landscape, JHEP 09 (2007) 128 arXiv:0708.2285.

[12] R. Blumenhagen, M. Cvetič and T. Weigand, Spacetime instanton corrections in $4 D$ string vacua - the seesaw mechanism for D-brane models, Nucl. Phys. B 771 (2007) 113 hep-th/0609191.

[13] L.E. Ibáñez and A.M. Uranga, Neutrino Majorana masses from string theory instanton effects, JHEP 03 (2007) 052 hep-th/0609213.

[14] R. Blumenhagen, M. Cvetič, F. Marchesano and G. Shiu, Chiral D-brane models with frozen open string moduli, JHEP 03 (2005) 050 hep-th/0502095.

[15] C.-M. Chen, V.E. Mayes and D.V. Nanopoulos, MSSM via Pati-Salam from intersecting branes on $T^{6} /\left(\mathbb{Z}_{2} \times \mathbb{Z}_{2}^{\prime}\right)$, Phys. Lett. B 648 (2007) 301 hep-th/0612087.

[16] C.-M. Chen, T. Li, V.E. Mayes and D.V. Nanopoulos, Towards realistic supersymmetric spectra and Yukawa textures from intersecti ng branes, Phys. Rev. D 77 (2008) 125023 arXiv:0711.0396].

[17] S. Förste and I. Zavala, Oddness from Rigidness, arXiv:0806.2328.

[18] D. Bailin and A. Love, Towards the supersymmetric standard model from intersecting D6-branes on the $\mathbb{Z}_{6}^{\prime}$ orientifold, Nucl. Phys. B 755 (2006) 79 Nucl. Phys. B 783 (2007) 176] hep-th/0603172.

[19] D. Bailin and A. Love, Almost the supersymmetric standard model from intersecting D6-branes on the $\mathbb{Z}_{6}^{\prime}$ orientifold, Phys. Lett. B 651 (2007) 324 [Erratum ibid. B 658 (2008) 292] arXiv:0705.0646].

[20] D. Bailin and A. Love, Constructing the supersymmetric Standard Model from intersecting D6-branes on the $\mathbb{Z}_{6}^{\prime}$ orientifold, arXiv:0801.3385.

[21] V. Bouchard and R. Donagi, On heterotic model constraints, arXiv:0804.2096.

[22] K.R. Dienes and M. Lennek, Fighting the floating correlations: expectations and complications in extracting statistical correlations from the string theory landscape, Phys. Rev. D 75 (2007) 026008 hep-th/0610319.

[23] S. Förste, C. Timirgaziu and I. Zavala, Orientifold's landscape: non-factorisable six-tori, JHEP 10 (2007) 025 arXiv:0707.0747.

[24] T. Kimura, M. Ohta and K.-J. Takahashi, Type IIA orientifolds and orbifolds on non-factorizable tori, Nucl. Phys. B 798 (2008) 89 arXiv:0712.2281. 
[25] D. Cremades, L.E. Ibáñez and F. Marchesano, SUSY quivers, intersecting branes and the modest hierarchy problem, JHEP 07 (2002) 009 hep-th/0201205.

[26] R. Blumenhagen, V. Braun, B. Körs and D. Lüst, Orientifolds of K3 and Calabi-Yau manifolds with intersecting D-branes, JHEP 07 (2002) 026 hep-th/0206038.

[27] R. Blumenhagen, L. Görlich and B. Körs, Supersymmetric $4 D$ orientifolds of type IIA with D6-branes at angles, JHEP 01 (2000) 040 hep-th/9912204.

[28] R. Blumenhagen, J.P. Conlon and K. Suruliz, Type IIA orientifolds on general supersymmetric $Z(N)$ orbifolds, JHEP 07 (2004) 022 hep-th/0404254.

[29] R. Blumenhagen, B. Körs and D. Lüst, Type I strings with F- and B-flux, JHEP 02 (2001) 030 hep-th/0012156.

[30] F. Gmeiner and G. Honecker, work in progress.

[31] R. Blumenhagen, D. Lüst and S. Stieberger, Gauge unification in supersymmetric intersecting brane worlds, JHEP 07 (2003) 036 hep-th/0305146.

[32] N. Akerblom, R. Blumenhagen, D. Lüst and M. Schmidt-Sommerfeld, Instantons and holomorphic couplings in intersecting D-brane models, JHEP 08 (2007) 044 arXiv:0705.2366.

[33] D.M. Ghilencea, L.E. Ibáñez, N. Irges and F. Quevedo, TeV-scale Z' bosons from D-branes, JHEP 08 (2002) 016 hep-ph/0205083.

[34] D. Lüst and S. Stieberger, Gauge threshold corrections in intersecting brane world models, Fortschr. Phys. 55 (2007) 427 hep-th/0302221.

[35] N. Akerblom, R. Blumenhagen, D. Lüst and M. Schmidt-Sommerfeld, Thresholds for intersecting D-branes revisited, Phys. Lett. B 652 (2007) 53 arXiv:0705.2150].

[36] P. Anastasopoulos, M. Bianchi, G. Sarkissian and Y.S. Stanev, On gauge couplings and thresholds in type-I Gepner models and otherwise, JHEP 03 (2007) 059 hep-th/0612234.

[37] R. Blumenhagen and M. Schmidt-Sommerfeld, Gauge thresholds and Kähler metrics for rigid intersecting D-brane models, JHEP 12 (2007) 072 arXiv:0711.0866.

[38] G. Honecker and T. Ott, Getting just the supersymmetric standard model at intersecting branes on the $\mathbb{Z}_{6}$-orientifold, Phys. Rev. D 70 (2004) 126010 [Erratum ibid. D 71 (2005) 069902] hep-th/0404055.

[39] R.A. Porto and A. Zee, The private higgs, arXiv:0712.0448.

[40] F. Gmeiner, Statistics in the landscape of intersecting brane models, arXiv:0710.2468.

[41] D. Lüst, P. Mayr, R. Richter and S. Stieberger, Scattering of gauge, matter and moduli fields from intersecting branes, Nucl. Phys. B 696 (2004) 205 hep-th/0404134].

[42] L.E. Ibáñez, F. Marchesano and R. Rabadán, Getting just the standard model at intersecting branes, JHEP 11 (2001) 002 hep-th/0105155. 